FEDERAL RESERVE BANK OF SAN FRANCISCO

WORKING PAPER SERIES

\title{
The Local Economic Impact of Natural Disasters
}

\author{
Brigitte Roth Tran \\ Daniel J. Wilson \\ Federal Reserve Bank of San Francisco \\ February 2023 \\ Working Paper 2020-34 \\ https://www.frbsf.org/economic-research/publications/working-papers/2020/34/
}

\section{Suggested citation: \\ Roth Tran, Brigitte, Daniel J. Wilson. 2023. "The Local Economic Impact of Natural Disasters," Federal Reserve Bank of San Francisco Working Paper 2020-34. https://doi.org/10.24148/wp2020-34}

The views in this paper are solely the responsibility of the authors and should not be interpreted as reflecting the views of the Federal Reserve Bank of San Francisco or the Board of Governors of the Federal Reserve System. 


\title{
The Local Economic Impact of Natural Disasters ${ }^{3}$
}

\author{
Roth Tran, Brigitte and Wilson, Daniel J. ${ }^{4}$
}

February 2023

\begin{abstract}
We study the dynamic responses of local economies after natural disasters in the U.S. using county-level data over the past four decades. We find disasters increase income total and per capita income over the longer run ( 8 years out). The effect is driven initially by an employment boost and in the longer run by higher wages. Over the longer run, house prices increase while population is roughly flat, especially in areas with inelastic housing supply, pointing to increases in housing demand, potentially reflecting increases in local amenities and/or productivity. The longer-run increase in income is largest for the most damaging disasters. It is especially apparent for hurricanes and tornados but absent for floods. The longer-run increase in income, which has fallen over time, is independent of recent disaster experience. State-level and spatial spillover analyses point to offsetting negative effects of local disasters on other counties within the region.
\end{abstract}

JEL Codes: R11, R15, Q54, Q58, H84

Keywords: natural disasters, local economic impacts of disasters, panel local projections

\footnotetext{
${ }^{3}$ We thank seminar participants of the Cornell University Applied Economics \& Policy Seminar, Richmond Fed Climate Change Economics Workshop, IMF 21st Jacques Polak Annual Research Conference, UC San Diego Environmental Economics Seminar, Federal Reserve System Regional Conference, the Federal Reserve Board Applied Micro Lunch series, the 2020 AEA meetings, the 2020 IIPF Annual Congress, the 2021 NBER Summer Institute Urban Economics Session, the 2021 Stanford SITE Housing and Urban Economics session, and the Santa Clara University Economics Seminar. We also thank Laura Bakkensen, Carolyn Kousky, Yuriy Gorodnichenko, and numerous colleagues at the Federal Reserve Board and Federal Reserve Bank of San Francisco for their helpful feedback. Nathan Ausubel, Olivia Lofton, Tessa Maddy, Stephanie Stewart, and Simon Zhu provided excellent research assistance. The analysis and conclusions set forth are those of the authors and do not reflect the views of the Federal Reserve Bank of San Francisco, the Federal Reserve System or its staff.

${ }^{4}$ Roth Tran: The Federal Reserve Bank of San Francisco. Email: brigitte.rothtran@sf.frb.org. Wilson: The Federal Reserve Bank of San Francisco. Email: daniel.wilson@sf.frb.org.
} 


\section{Introduction}

Natural disasters in the United States have become more frequent and costly in recent decades (see Figure 1(a)). While increased development and population growth in disaster-prone areas have played a role (Rappaport and Sachs 2003), climate change is often cited as an important driver of these trends (USGCRP 2017). Consensus climate change projections indicate that the frequency and severity of many types of disasters are likely to rise further in the decades ahead. ${ }^{1}$ In light of these trends, it has become increasingly important for policymakers to understand the economic impacts of natural disasters, both over the short run and over the longer run. Local policymakers need to anticipate how disasters will affect local employment, earnings, population, and property values, all of which can directly impact local tax revenues. ${ }^{2}$ National policymakers need to understand the impacts of disasters to make effective investments in resilience and support better budgeting (CEA 2022). For instance, the economic impacts of severe weather events tied to climate change are an important input into estimates of the social cost of carbon (Carleton and Greenstone (2021)), which plays a key role in U.S. government policy.

Despite the importance to policymakers and an abundance of studies, there is little consensus on what the dynamic impacts of natural disasters are for local economic outcomes. Hsiang and Jina (2014) provide a useful frame of reference for thinking about the potential impacts. They present a schematic, which we reproduce in Figure 2, that depicts four potential paths illustrating that long-run economic activity may end up below, at, or even above pre-disaster trends. Any one of these paths can be rationalized by economic theory (Botzen, Deschenes, and Sanders (2019)). For instance, destruction of local wealth and capital stocks can lead to business closures and an exodus of firms and investment, thereby driving long-run declines in local income. Then again, the inflow of aid and insurance payouts to areas with attractive locational fundamentals may fund reconstruction and

\footnotetext{
${ }^{1}$ For instance, the recent Climate Science Special Report (Fourth National Climate Assessment: Volume 1) from the Congressionally-mandated U.S. Global Change Research Program (USGCRP 2017) concludes that "the frequency and intensity of extreme high temperature events are virtually certain to increase in the future as global temperature increases (high confidence). Extreme precipitation events will very likely continue to increase in frequency and intensity throughout most of the world (high confidence)." The report goes on to note that these trends will result in increased frequency and severity of disaster types such as droughts, fires, and floods that are associated with high temperatures and swings in precipitation.

2 See, for example, "Harvey-struck Texas counties face blow to property tax revenues" (Reuters 2017).
} 
stimulate other investments that could boost local productivity and income in the longer run. Expectations regarding future risk, insurance, and aid could influence willingness to invest in an area after a disaster and determine longer-run recovery of economic activity. Hence, economic theory alone is of limited use for predicting the likely economic impact of a natural disaster.

As discussed in the following section, there is an extensive body of empirical work estimating the impacts of natural disasters. Reflecting the diverse possibilities supported by economic theory, results from the empirical literature are wide-ranging, with support for each of the paths in Figure 2. For U.S. policymakers seeking insights on the broader effects of natural disasters for local economies this literature has three shortcomings. First, most prior studies estimate only contemporaneous or short-run effects, which may be very different than longer-run effects. ${ }^{3}$ For example, the fact that disaster recovery funds from aid programs like Community Development Block Grants can take years to reach affected areas suggests that short-run impacts may not reflect longer-run dynamics. ${ }^{4}$ Second, much of the prior literature has focused on the impacts of hurricanes or other specific types of disasters, often using country-level outcome data. Hurricanes - or any one disaster type — may not be representative of disasters more broadly. Third, insights developed from studies using country-level data may not apply to local outcomes within a country like the U.S.

In this paper, we address these concerns using nearly four decades of data on U.S. counties to estimate both the shorter and longer-run local dynamic impacts of a broad range of natural disasters on economic growth. We assess impulse response functions for personal income per capita as well as several key underlying components and investigate the mechanisms underlying those impacts. We also examine heterogeneity, adaptation, and spatial spillover effects, allowing us to directly assess questions like whether responses to one type of disaster like hurricanes apply more broadly.

We start by asking what is the average response of local income to natural disasters? We focus on personal income, which is very highly correlated with GDP and, unlike GDP, is

\footnotetext{
${ }^{3}$ Botzen, Deschenes, and Sanders (2019) raised this last concern in a recent literature review, saying "more research is needed on long-term impacts (e.g., beyond 5 years) of natural disasters."

${ }^{4}$ In an analysis of the Community Development Block Grant Disaster Recovery Program, Martin (2018) finds that "Within the same sample of grants, housing activities on average take 4.7 years to complete from the time a disaster is declared, and overall grants (including non-housing activities) on average take 5.1 years." (p. 5)
} 
available at the county level back to $1980 .{ }^{5}$ We find robust evidence of long-lasting increases in local personal income per capita following natural disasters in the U.S. Indeed, of the four hypotheses depicted in Figure 2, the estimated response is somewhere between the "build back better" and "creative destruction" patterns. Specifically, our results point to an economically and statistically insignificant initial decline, followed by a recovery to a level of income per capita that is $0.6 \%$ above the baseline trend eight years after the disaster.

We then ask what are the key components driving this increase in income per capita? We find that it is initially explained by a temporary boost in employment, especially in the construction sector. However, over the longer run, the increase in personal income can be largely traced to average weekly wages. We also look at the responses of house prices and population to disasters, which help shed light on the economic mechanisms underlying the income per capita response. We find a longer-run increase in house prices, starting about five years after the disaster, and roughly no change in population. In standard spatial equilibrium models such as Hsieh and Moretti (2019), an increase in house prices with no change in population indicates that housing supply is relatively inelastic for the average county, such that increases in local demand after disasters push up house prices rather than population. Indeed, when we estimate the disaster IRF separately for high and low housing supply elasticity counties, using Saiz's (2010) estimates, we find house prices rise only in lowelasticity counties while population rises in (and only in) high-elasticity counties.

Next, we consider the extent to which the average post-disaster response of local income applies across different contexts. We uncover considerable heterogeneity in this response along several key dimensions. First, we find that while hurricanes and tornados result in longer-run increases in income per capita, the effects of floods, severe storms, extreme winter weather, and fires are small or statistically insignificant. Second, we find that the rise in income per capita actually increases with disaster severity. ${ }^{6}$ Third, we segment counties by

${ }^{5}$ There are three main conceptual differences between GDP and personal income at the county level as defined by the U.S. Bureau of Economic Analysis: (1) personal income includes government transfers, while GDP does not, (2) GDP includes corporate income while personal income does not (though it does include corporate income distributed to shareholders via dividends and interest), and (3) GDP is based on place of work, while personal income is based on place of residence. One implication is that our results on personal income generally will not reflect any post-disaster losses (or gains) to corporate profits. For example, Kruttli, et al (2020) have found that firms affected by hurricanes experience significant uncertainty, with significant outperformance and underperformance in returns for affected firms several months after landfall.

${ }^{6}$ In our preferred specification, we measure severity using monetary damages per capita, data on which are comparable and available for all types of disasters. We also report results, just for hurricanes, using wind speed as an 
initial income and find that, while the income per capita boost in the first few years after a disaster appears to be concentrated in the richest quartile of counties, the longer-run increase is largely independent of initial income.

We next turn to the issue of adaptation. Economic agents-households, firms, and policymakers - may make adaptive investments and behavior changes if they anticipate an increase in disaster frequency or severity going forward. We take two approaches to examine the extent of adaptation. First, consistent with local adaptation, we find that counties with more historical experience with disasters see larger increases in personal income over the longer run. Second, we test whether the average dynamic response of income per capita to disasters has changed over time. While the near-zero contemporaneous effect and longer-run increase are roughly stable over time, the dynamics in between appear to have changed somewhat. Specifically, the increase in income per capita seems to have become more delayedin other words, what used to be a strong increase in the medium run ( 3 to 5 years out) has fully dissipated over time.

Finally, we use two methods to examine spatial spillovers to see how disaster effects propagate to other counties of varying distances away. First, using a spatial lagmethodology, we show that nearby counties experience a medium-run boost to personal income but are largely unaffected over the longer-run, consistent with residents of nearby counties participating in recovery efforts. Further away counties, on the other hand, see a decline in personal income over all horizons, which could be explained by resources being redirected to counties directly affected by disasters. Aggregating the own-county effects with the spatial lag effects, we find that the longer-run income effect for a region-i.e., all counties within 600 miles of a disaster's epicenter-is small and statistically insignificant.

Second, we examine state level outcomes using a continuous treatment measure that aggregates county disaster indicators. While the dynamic pattern is qualitatively similar to our baseline per capita income result at the county level, the state level impacts are small and statistically insignificant. This evidence that the longer run income per capita (p.c.) response to

alternative measure. Those results also show larger income p.c. increases for the most severe disasters in the shortto medium run, though smaller increases over the longer run. We discuss the relative merits of measuring severity based on damages versus physical metrics like wind speed in Section IV. 
disasters becomes more negative at coarser levels of aggregation may help explain the negative longer-run effects of disasters found in some prior studies based on country-level data.

Our findings have several important policy implications. First, for local policymakers, the finding that employment, and perhaps personal income (for which we don't have high-frequency data), temporarily drop immediately after a disaster suggests they may need to plan ahead — for example, with larger rainy-day funds - to better manage post-disaster tax base declines. Second, the heterogeneity in outcomes suggests that we must exercise caution in extrapolating from results based on specific events, contexts, or time frames. Finally, if local areas really do "build back better" after disasters, an important question for future research is how much of this relies on government aid and other policies. As climate change leads to more frequent and severe disasters, allocating resources to rebuilding disaster-prone areas — which may encourage additional investment there - may not be a sustainable approach.

The remainder of the paper is organized as follows. In the next section, we discuss key findings from prior research. In section III, we discuss the economic channels by which disasters could impact local economies. Then in section IV, we describe the data we use both for disasters and to measure economic activity. We follow this with a discussion of our methodology in section V. In section VI, we present our baseline results. Section VII examines heterogeneity, adaptation, and spatial spillover effects. Section VIII concludes.

\section{Literature}

Previous research on the dynamic economic effects of disasters generally has focused on national aggregate outcomes, on narrow local outcomes, or on case studies of particular disasters. $^{7}$ As mentioned above, Hsiang and Jina (2014) use cross-country panel data to study the dynamic impact of cyclones on national GDP per capita, finding a long-lasting decline. ${ }^{8}$ Yet, a number of other cross-country studies find less negative, or even positive, effects of disasters on national income for high-income countries like the U.S. Lackner (2019) shows that eight years after impact, earthquakes reduce per capita GDP for low- and middle-income

\footnotetext{
${ }^{7}$ See Botzen, Deschenes, and Sanders (2019) for a recent literature review.

${ }^{8}$ See also Noy (2009), who shows that natural disasters contemporaneously reduce national GDP on average and more so in countries that are poorer, less open, or less educated.
} 
countries, but may boost it for high-income countries. Similarly, von Peter, von Dahlen and Saxena (2012) find that while the average response of national GDP per capita to natural disasters is negative, the response to well-insured disasters (which are predominately in highincome countries) "can be inconsequential or positive for growth over the medium term as insurance payouts help fund reconstruction efforts." In another cross-country analysis, Cavallo, Galiani, Noy, and Pantano (2013) find that when they include controls for political revolutions that occurred after natural disasters, even the most severe disasters appear to have no significant effect on economic growth. Another recent cross-country study, Sawada and Sachs (2019), find that natural disasters and wars have positive long-run effects on per capita GDP growth.

There have also been a number of studies of disasters' impacts on local economies in the U.S., though these studies generally consider only on short-run impacts. Strobl (2011) focuses on coastal U.S. counties and finds that annual per capita income growth falls significantly in the year of the hurricane but returns to the pre-hurricane growth rate in the following year. This growth pattern implies an initial reduction in the level of income, relative to the no-disaster counterfactual, that is not fully recovered in the subsequent year, consistent with the "no recovery" scenario depicted in Figure 2. Belasen and Polacheck use data on counties in Florida to examine the short-run effects of hurricanes, finding a negative short-run impact on employment but a positive impact on earnings. ${ }^{9}$ By contrast, Groen, Kutzbach, and Polivka (2020) perform a careful longitudinal study of workers affected by Hurricanes Katrina and Rita in 2005, finding substantial long-term gains in earnings, driven largely by higher wages. Similarly, Deryugina, Kawano, and Levitt (2018) follow over time the income tax returns of individuals that were in areas hit by Hurricane Katrina and find that by 3 years after the hurricane, average labor income for these individuals is higher than that of a control group of unaffected individuals.

Another within-U.S. study that is closely related to ours is Boustan, Kahn, Rhode, and Yanguas (2020). Using county-by-decade panel data from 1940 to 2010, they estimate the contemporaneous (i.e., within same decade) effects of severe disasters on several economic outcomes. They find that counties affected by the most severe disasters experience higher net

\footnotetext{
${ }^{9}$ Similarly, Barattieri, et al. (2023) finds negative short-run employment effects of hurricanes for counties in Puerto Rico.
} 
out-migration, higher poverty rates, and lower house prices. In this paper, we similarly find significant medium- to longer-run (up to 8 years out) increases in net out-migration and declines in population and house prices after very severe disasters. We also uncover two related results. First, there is actually a strong positive response of house prices after severe disasters in the shorter-run, lasting about 2-3 years. ${ }^{10}$ This temporary boost in prices could be due to a temporary drop in housing supply caused by disaster destruction that combines with stable or increasing demand for workers in the area during recovery and rebuilding efforts. Second, we find a different pattern for less severe disasters - i.e., those with damages per capita below the $90^{\text {th }}$ percentile for all disasters. For these disasters, the longer-run response of both population and house prices is slightly positive, yielding an average home price response that is positive in our 8-year horizon.

There is a broad literature examining effects of specific types of natural disasters or even specific events on particular sets of outcomes. For example, examining the first three years thereafter, McCoy and Walsh (2017) find that wildfires in Colorado yield short-lived declines in house prices, while Bin and Landry (2013) find that hurricane flooding causes temporary declines in house prices in affected areas. Separately, there are a fairly large number of detailed case studies of specific disasters. Prominent examples include Vigdor (2008), Hornbeck (2012), Gallagher and Hartley (2017), Kirchberger (2017), Deryugina, Kawano, and Levitt (2018), and Groen, Kutzbach, and Polivka (2020).

\section{Economic Channels}

The dynamic effects of natural disasters on local economic outcomes is far from clear $a$ priori. Disasters can be thought of as negative shocks to public and private capital stocks and to household wealth. Indeed, in our empirical analysis we exclude from our definition of disasters any events for which there are no documented monetary damages. The literatures on economic growth and spatial equilibria provide useful insights as to the potential local economic effects of such damage shocks in the short- and longer-run.

In a standard Solow growth model, a one-time capital depreciation shock leads to higher investment and output growth as the economy transitions back to steady state. Similarly, in a

\footnotetext{
${ }^{10}$ This result is consistent with Graff Zivin, Liao, and Panassié (2020)'s finding that home prices are elevated for up to 3 years after hurricanes in Florida, though they do not look beyond 3 years.
} 
standard Neoclassical model of labor supply, a one-time negative wealth shock increases the marginal utility of consumption, inducing households to increase labor supply, increasing employment and income all else equal. Thus, these models suggest disaster shocks could potentially increase employment, income, and related measures of local economic activity in the short- to medium-run.

The classic local labor markets model of Rosen (1979) and Roback (1982) (and its more recent articulations such as Hsieh and Moretti (2019)) points to the central roles of productivity and amenities, and their effects on population movements, in determining the longer-run impact of local shocks on the spatial equilibrium of economic activity. Shocks that increase local amenities increase local housing demand, which in turn puts upward pressure on population and house prices. The extent to which shocks to local housing demand manifest in higher population versus higher house prices depends on the elasticity of housing supply. Shocks that increase local productivity increase local labor demand, which in turn puts upward pressure on employment and wages. The extent to which shocks to local labor demand manifest in higher employment versus higher wages depends on the elasticity of labor supply, including the migration margin. In particular, two hypotheses of the local labor markets model are that (1) house prices should respond more to disasters in areas with relatively inelastic housing supply and (2) population (assumed to move with housing quantity) should respond more to disasters in areas with relatively elastic housing supply. In Section VI.C., we test and find strong empirical support for both of these hypotheses.

One might expect natural disasters to negatively affect both productivity and amenities in the short-run. However, after that immediate negative impact, productivity and amenities may recover to their pre-disaster levels over the medium- to longer-run. For example, Davis and Weinstein (2002) found that within 15 years of the extensive bombing of some Japanese cities in World War II, the spatial population distribution had fully recovered. Yet, natural disasters can differ from war destruction shocks in at least one important way. A natural disaster may increase the probability of future disasters perceived by local producers and residents, reducing the attractiveness (anticipated productivities and amenities) of the area. If so, the disaster may lead to (a) permanently lower economic activity in the area and (b) a more rapid transition (e.g., investment, employment, and construction) to the new lower steady state. 
In contrast, in some settings one might expect economic activity after damaging shocks to more than fully recover. In particular, shocks could improve productivity and amenities over the medium- to longer-run if capital and housing stocks are "built back better." Hornbeck and Keniston (2017) develop a theoretical micro-foundation for the build back better scenario and provide supporting empirical evidence from the 1872 Great Fire of Boston. According to their model, underinvestment in local buildings can occur in growing urban areas because an individual landowner does not internalize the increase in property values of other local buildings that occurs when the landowner improves their own property. A disaster, such as the Great Fire, causes many landowners to simultaneously improve/modernize their properties, increasing the values of all local properties, even those undamaged by the disaster. Such a process may be facilitated by insurance and government aid, especially in advanced economies like the U.S. An interesting, testable implication of the Hornbeck and Keniston (2017) model is that property values should increase after a disaster only in growing localities because those are the localities subject to underinvestment. We test and find support for this hypothesis for the disasters in our sample in Section VI.C.

Finally, natural disasters in the U.S. typically trigger large amounts of insurance payouts and government aid. ${ }^{11}$ These external fiscal injections could have positive or negative net effects on local economic activity. On the one hand, aid may spur economic activity through a government spending multiplier. While estimates of the size of the government spending multiplier vary widely, the literature generally has found large multipliers on employment and income in local areas from federal spending that is not financed by local taxation (i.e., local windfall spending). See, for example, Shoag (2013), Wilson (2012), Chodorow-Reich, Feiveson, Liscow, and Woolston (2012), and Chodorow-Reich (2019) for state-level evidence and Suárez Serrato and Wingender (2016) for county-level evidence. On the other

\footnotetext{
${ }^{11}$ The National Flood Insurance Program (NFIP) payouts averaged \$2.2 billion a year from 2006-2016. FEMA's Public Assistance (PA) program, which issues grants to state and local governments to repair or rebuild public infrastructure, averaged over $\$ 3.3$ billion a year in grants over the same period. In terms of individual aid, in the U.S. FEMA provides grants to individuals for temporary housing and other needs through its Individual Assistance programs, which includes the Individual and Household Program (IHP). The Small Business Administration (SBA) makes loans to qualified individuals, households, and businesses to help cover uninsured or underinsured property losses. These two individual transfer and loan programs averaged about $\$ 370$ million per year from 2006-2016. Disasters may also trigger significant transfer payments from non-disaster safety-net programs such as Unemployment Insurance, Temporary Assistance for Needy Families, Medicaid, and the Earned Income Tax Credit. Transfers from these programs increase after a disaster as more households in the affected area qualify, as found in Deryugina (2017) for hurricanes.
} 
hand, aid received by households displaced from their housing — especially aid that is not required to be used for rebuilding - may facilitate household relocation away from affected areas. In particular, while Small Business Administration (SBA) disaster loans need to be repaid and rely on the homes that the funds are intended to repair as collateral, monies received by households from FEMA Individual Assistance aid and NFIP payouts have fewer strings attached.

Given the theoretical ambiguity of the local economic effects of disasters, we are agnostic a priori as to the sign and magnitude of disasters' effects on local income, employment, wages, and other outcomes. We empirically trace out the dynamic effect of disasters on local economic activity - that is, the net effect from all potential channels — over time. We study a broad range of economic outcomes that should capture the effects of the shocks to local labor demand and supply and to locational fundamentals like productivity and amenities.

\section{Data}

We use data on disasters and a variety of economic outcomes, which we describe below. Appendix Table A1 summarizes the sources and treatment of the dependent variables, while Appendix Table A2 shows summary statistics. Note that all nominal monetary variables are deflated by the BLS Current Price Index - All Urban Areas (CPI-U) in constant 2017 dollars.

\section{A. Natural Disasters}

Our primary data source for disasters is FEMA's real-time administrative Disaster Declarations Summary dataset. Although FEMA disaster declarations go back to 1953, we only estimate IRFs for disasters that occurred between 1980 and 2017 based on the availability of our economic outcome data. We focus on natural disasters that received a "Major Disaster" Presidential declaration according to the FEMA data. ${ }^{12}$ A FEMA disaster declaration is generally initiated when a state government issues a request to FEMA. FEMA sends a team to the disaster area to perform a Preliminary Damage Assessment, using drone, satellite, and civil air imagery as well as site visits to determine, for each affected county, whether the damage is extensive enough to warrant a major disaster designation and, if so, for what types of federal government

\footnotetext{
${ }^{12}$ Given our focus on natural disasters, we exclude declarations due to terrorism or toxic substances.
} 
assistance the county is eligible. ${ }^{13,14}$ Some types of assistance go to individuals and households such as FEMA Individual Assistance (IA) aid and Small Business Administration (SBA) loans. Other types go to state and local governments such as FEMA Public Assistance (PA) grants for infrastructure repair, FEMA Hazard Mitigation Grant Program (HMGP) funds to lessen the effects of future disaster incidents, and Community Development Block Grant Disaster Recovery (CDBG-DR) funds from the U.S. Department of Housing and Urban Development that are meant for long-term economic recovery and development.

The resulting sample of natural disasters covers much of the country, with 96 percent of counties experiencing at least one FEMA disaster declaration between 1980 and 2017.

Figure 1(b) shows for each county the number of years from 1980 to 2017 in which it had a disaster. The modal county experienced disasters in eight of the 38 years of this period.

To examine heterogeneity of effects by disaster type, we use FEMA disaster type categories with some modifications. We designate as a hurricane any FEMA disaster declaration containing "hurricane" in the declaration title or categorized by FEMA as a hurricane. We then designate as a flood any remaining disaster classified by FEMA as a flood or containing "flood" (but not "hurricane") in its title. ${ }^{15}$

\section{B. Income}

We use annual county level data on personal income and its components from the Regional Economic Information System (REIS) of the Bureau of Economic Analysis for 1980 through 2017 (see BEA 2017). In addition to total personal income, we examine wage and salary income, government transfers (and loans), and poverty rates. We describe these data in Appendix B.

\section{Employment and Average Weekly Wages}

Our data on employment and average weekly wages by county come from the Quarterly Census of Employment and Wages (QCEW), as used and described in detail in Wilson (2017). The QCEW is compiled by the Bureau of Labor Statistics (BLS) based on state

\footnotetext{
13 Source: author conversations with FEMA staff.

${ }^{14}$ As detailed in Lindsay and Reese (2018) from the Congressional Research Service, "[e]ach presidential major disaster declaration includes a 'designation' listing the counties eligible for assistance as well as the types of assistance FEMA is to provide under the declaration.

15 The geographic exposure to disasters varies significantly by disaster type (see Appendix Figure A1).
} 
Unemployment Insurance administrative records. Nearly all private nonfarm employers in the U.S. are required to report monthly employment counts and quarterly wages of their employees to their state Unemployment Insurance agencies. Employment covers "all full- and part-time workers who worked during or received pay (subject to Unemployment Insurance wages) for the pay period which includes the 12th day of the month." We separately examine effects for total nonfarm employment and construction employment (category 1012). ${ }^{16}$ Our data on total nonfarm employment and wages covers the period January 1980 to December 2016, while the construction employment data start in January $1990 .{ }^{17}$

The BLS calculates average weekly wages (AWW) "by dividing quarterly total wages by the average of the three monthly employment levels (all employees, as described above) and dividing the result by 13 , for the 13 weeks in the quarter." 18 Note that AWW reflect both hourly wages and the number of hours worked per week.

\section{House prices}

We use the CoreLogic Home Price Index (HPI), available by county at a quarterly frequency from 1980Q1 to 2016Q4, to measure house prices. The index is based on transaction prices of repeated home sales. Repeated-sales price indices have the advantage of reflecting price changes of individual houses holding fixed the permanent characteristics of the houses and are therefore independent of changes in the composition of houses in an area. However, a natural disaster can seriously affect the characteristics of a given house. For instance, unrepaired damage will negatively impact a house's value, while improvements made through repairs and rebuilding may increase its quality. This potential for changing home characteristics should be kept in mind when interpreting our house price results.

In addition, we use the housing supply elasticity data from Saiz (2010), which is based on topographical characteristics of metro areas.

\footnotetext{
${ }^{16}$ Due to concerns about data quality, when estimating IRFs for construction employment, we drop counties with more than 5 months of missing or zero construction employment.

${ }^{17}$ Our employment and wages data cover "nonfarm" employment and so exclude any employment in agriculture, ranching, fishing, and hunting.

18 https://www.bls.gov/news.release/cewqtr.tn.htm.
} 


\section{E. Population}

Estimates of annual population by county were obtained from the Census Bureau for 1980 through 2017 and reflect the population in each county as of July 1 of each year.

\section{Methodology}

To see how local economies are affected by natural disasters, we estimate impulse response functions (IRFs) of economic outcomes with respect to disaster shocks. We use a variant of the Jordà (2005) local projection method, modified for panel data, as our baseline specification. We then build on that specification to explore heterogeneity in disaster effects.

In our baseline specification, we estimate the following equation for a series of horizons $h \geq 0$ :

$$
y_{c, t+h}-y_{c, t-1}=\beta^{h} D_{c, t}+X_{c t}^{\prime} \gamma^{h}+\alpha_{r(c), t}^{h}+\alpha_{c, m(t)}^{h}+\epsilon_{c, t+h} .
$$

$y_{c, t}$ is an economic outcome of interest in county $c$ in period $t$, measured in logs. Our dependent variable is thus the change in the log outcome (approximately equal to the percentage change in the outcome) from the period before the disaster to $h$ periods after the disaster. Appendix Table A1 outlines how the outcome variables are measured in our analyses. $D_{c, t}$ is the key treatment variable, equaling one if the county experienced a disaster in period $t$ and zero otherwise, as described in Section IV.A. The series of $\beta^{h}$ are the IRF coefficients of interest. $\boldsymbol{X}_{\boldsymbol{c} t}$ is a vector of control variables with parameters $\gamma^{\boldsymbol{h}}$. Specifically, $\boldsymbol{X}_{c t}^{\prime} \gamma^{\boldsymbol{h}}$ is defined as

$$
\boldsymbol{X}_{c t}^{\prime} \gamma^{\boldsymbol{h}} \equiv \sum_{\substack{\tau=-p, \tau \neq 0}}^{h} \delta^{\tau h} D_{c, t+\tau}+\rho^{h} \Delta y_{c, t-1, t-k}
$$

The first term in equation (2) controls for other disasters that may have hit the same county either before the current disaster (up to $p$ periods prior) or between the current disaster and the horizon of interest $(h) .{ }^{19,20}$ This ensures that the estimated IRF from a

\footnotetext{
${ }^{19}$ Though not shown in equation (2) for tractability, when $h<0$ we still include the $p$ lags indicating whether disasters occurred before period 0 .

${ }^{20}$ For monthly outcomes, due to computational demands, we control for 12-month aggregate indicators for the leads and lags of disasters.
} 
disaster is not contaminated by either lingering effects of past disasters or effects of other disasters that happen to occur between the current disaster and horizon $h .^{21}$

The second term in equation (2) $\left(y_{c, t-1}-y_{c, t-k}\right)$ explicitly accounts for a pre-trend in the outcome variable. Because the dependent variable is the sum of period-by-period changes in the (log) outcome over the post-disaster timeframe up until horizon $h$-that is, $y_{c, t+h}-y_{c, t-1} \equiv$ $\sum_{i=0}^{h} \Delta y_{c, t-i}$ - this pre-trend term is a lag of the dependent variable with a different time horizon. We measure this pre-trend over the prior three years, so $k$ equals 3,12 , or 36 depending on whether the outcome variable is annual, quarterly, or monthly. ${ }^{22,23}$

We include region-specific time fixed effects $\alpha_{r(c), t}^{h}$ to absorb any regional or national shocks that may have coincided with disasters. To control for county-level heterogeneity and seasonality, we also include county-by-calendar month (quarter for quarterly frequency data) fixed effects $\alpha_{c, m(t+h)}$. For annual outcomes, this amounts to a simple county fixed effect. In addition, we report standard errors that allow for two-way clustering at the county and state-bytime levels. Clustering by county allows for arbitrary serial correlation. State-by-time clustering allows for cross-sectional/spatial correlation of errors within each state such as those due to state government policies.

Note that the key identifying assumption in equation (1) is that the disaster indicator is orthogonal to the error term, $\epsilon_{c, t+h}$. In other words, we assume that FEMA disaster declarations are exogenous with respect to any factors that affect the trajectories of our outcomes that are unobserved or omitted from equation (1).

In Section VII, we extend this baseline specification in several directions, exploring heterogeneous disaster effects, adaptation, and spatial spillovers.

${ }^{21}$ We note that in practice in our sample, the inclusion/exclusion of these intervening disaster dummies has virtually no effect on our results.

22 The choice of $k$ involves a trade-off: higher values of $k$ may provide a better forecast of the counterfactual nodisaster trend in the outcome between time 0 and $h$ but will also reduce the sample size available for any given $h$ regression.

${ }^{23}$ We could alternatively control for the counterfactual no-disaster trend by including a county-specific time trend, which would entail no loss of regression observations from the beginning of the sample. The downside of this approach is that a county's post-disaster time trend could itself be impacted by the disaster, making it a "bad control" (Angrist and Pischke 2009, Goodman-Bacon 2021). Nonetheless, to assess robustness, in Appendix Figure A2, we provide alternative IRF results for each of our main outcome variables whereby we replace the pre-trend term in equation (2) with a county-specific time trend (i.e., an interaction between county fixed effects and the time variable). 


\section{Baseline Results}

We now present our baseline IRF estimates, which come from estimating $\beta^{h}$ in equation (1) above. The results are shown in Figure 3. The shaded areas around the coefficient estimates represent 90 and 95 percent confidence intervals, calculated based on errors that are robust to heteroskedasticity and clustering by county and year-by-Census region (to account for serial and spatial correlation). Recall that these IRFs should be interpreted - in line with an average treatment effect interpretation - as estimates of the average cumulative difference between the actual outcome for a county hit by a disaster and the counterfactual outcome for that county had it not been hit by a disaster. In other words, a point estimate on the horizontal zero line in the IRF graphs does not mean that the level of the outcome variable is equal to its pre-disaster $(t-1)$ level, but rather that it is equal to our estimate of what it would have been in a no-disaster counterfactual. This no-disaster counterfactual reflects region-specific time fixed effects and county fixed effects as well as the controls in equation (2). For monthly and quarterly outcomes, the county fixed effects are also interacted with calendar month or calendar quarter dummies in order to control for seasonality.

\section{A. Personal Income Per Capita}

Figure 3 panel (a) shows the estimated IRF for personal income per capita (p.c.). The estimated contemporaneous response is negative but economically and statistically insignificant. As of one year after a disaster, income p.c. is about $0.2 \%$ higher than it would have been otherwise. Income p.c. remains about that much higher for the next several years and then increases more around 6 years out. As of 8 years out, income p.c. is estimated to be a little over $0.6 \%$ above where it otherwise would have been. Recalling the hypothetical scenarios in Figure 2, these baseline results on income p.c. lie somewhere between the "build back better" and "creative destruction" scenarios.

Although these results establish that the longer run average income increases in counties directly affected by natural disasters, they do not tell us whether income increases across the income distribution or whether these average effects are instead driven, for example, by the top end of the distribution which would imply a rise in inequality. Thus, before turning to the key components driving the average effects on personal income, we briefly report results on the response of the poverty rate to natural disasters. Shown in Appendix Figure A3, we find a positive but statistically insignificant longer-run effect of average disasters on the poverty 
rate. If the personal income increase were experienced equally by all parts of the income distribution, we would expect the poverty rate to decline as some see their income rise above the poverty threshold. The absence of a decline in the poverty rate suggests that despite an increase in average personal income per capita, local inequality may rise after natural disasters.

\section{B. Robustness}

Before investigating impacts of disasters on other outcomes, we assess the robustness of these results on personal income per capita to alternative specifications, sample restrictions, and disaster measurement.

We consider alternative specifications for modeling the counterfactual county-specific time trends in absence of a disaster treatment. As shown in equation (2), our baseline specification controls for the trend in the outcome variable over the prior three years, implicitly assuming that, conditional on the other controls, this trend would have continued in the absence of a disaster. Our first alternative specification drops this pre-trend variable. The IRF for income per capita based on this specification is shown in Appendix Figure A4, panel (b). The baseline IRF is shown in panel (a) for comparison. The results are nearly identical. Next, we replace this pretrend variable with a county-specific linear time trend (i.e., the time variable interacted with county fixed effects). We do not use this specification as our baseline because of the a priori concern that the estimated full-sample time trend could absorb the treatment effect itself. That is, the time trend is endogenous with respect to the disaster treatment because a disaster may well alter a county's income per capita trend. The results of this specification are shown in panel (c) and yield a positive, albeit somewhat smaller, longer-run response of income per capita to a disaster shock. ${ }^{24}$ The smaller positive impact is consistent with the estimated trend absorbing some of the true longer-run positive response. Lastly, rather than controlling just for the threeyear log change in income per capita as we do in the baseline, panel (d) shows the results where we control for the annual change in each of the prior three years. The resulting IRF is nearly identical to the baseline.

Next, we explore the sensitivity of our results to outlier counties that experience disasters either very rarely or very frequently. One a priori concern with our analysis based on using all U.S. counties is that counties that never or rarely experience disasters may not represent a valid

\footnotetext{
${ }^{24}$ The robustness to including county-specific time trends is true for the other outcomes as well, as shown in Appendix Figure A2.
} 
control group to compare against other counties that are treated (i.e., experience a disaster) more often. To assess this concern, we estimate the disaster IRF for income per capita repeatedly, first dropping counties that have never experienced a disaster (in our 1980-2017 sample period) - that is, only including counties with at least one disaster - then only including counties that have experienced at least two disasters, and so on up to 15 disasters. In panel (a) of Appendix Figure A5, we plot the estimated longer-run disaster impact on income per capita, $\hat{\beta}^{8}$ (dots), as well as the sample size (bars), for each restricted sample. The coefficient is generally stable across the samples, suggesting that including non-disaster-prone counties does not cause any notable bias. As we increasingly limit the sample to only very disaster-prone counties, the confidence interval widens such that the estimated longer-run impact becomes statistically insignificant for samples excluding counties with fewer than 10 disasters. However, this appears to be explained more by the shrinking of the sample size rather than a decline in point estimates. A similar concern is that our estimates are biased due to outsize influence of counties that experience disasters very frequently, which may be inherently different in other unobserved ways. In panel (b) of Appendix Figure A5, we show a plot similar to that in panel (a), except here we sequentially drop counties that experience more than a given number of disasters. The coefficient point estimates are again generally stable. Only when we limit the sample to the small set of counties that experienced no more than three disasters during our sample period do we see the point estimate drop. However, these estimates are quite noisy as indicated by the wide confidence intervals and are based on very small samples of counties that account for only up to about 5 percent of total population and are themselves likely to be unrepresentative of the typical county. ${ }^{25}$

Another potential concern with the baseline results is measurement error in the FEMA disaster declarations. In some instances, FEMA declarations cover all counties in an affected state even when only a portion of counties were physically affected. Hence, as a robustness check, we exclude the roughly $6 \%$ of FEMA-declared disasters with zero reported damages according to data from the Spatial Hazard Events and Losses Database for the United States (SHELDUS). The SHELDUS database is based on data from the NOAA Storm Database,

\footnotetext{
${ }^{25}$ Results are not shown for samples including only counties with at most 1 or 2 disasters because these samples are extremely small. They include only 2.2 and 5.7 percent of counties and 0.1 and 2.5 percent of total population, respectively.
} 
which are in turn based on reports from insurance companies, media, and other sources. We aggregate damages within the county over all events occurring during a month to estimate total disaster damages by county and month. ${ }^{26} \mathrm{We}$ then use Census population data to estimate per capita damages. The results are shown in Appendix Figure A6, panel (b). Comparing this IRF to the baseline result, which is reproduced in panel (a), we see that the results are very similar.

\section{Key Components Underlying Income Per Capita}

We next estimate the disaster IRFs for outcomes that are key components driving the longerrun positive response of income per capita to disasters. We start with the estimated IRF for total nonfarm employment, which is estimated at a monthly frequency. The results are shown in panel (b) of Figure 3. Consistent with an initial disruption in activity, employment falls sharply, by about $0.08 \%$, in the month of the disaster. Average monthly employment growth in our sample is approximately $0.09 \%$, so this initial impact amounts to wiping out nearly all of that month's employment growth. The initial decline carries over into the next month, but then rises significantly over subsequent months for an extended recovery period, with employment peaking around one year out. After this recovery period, employment gradually returns to the no-disaster counterfactual. As of eight years out, the point estimate suggests modestly higher employment of about $0.3 \%$ but it is not statistically significant.

To get a better sense of the extent to which the overall employment response is driven by recovery and rebuilding efforts, we look at the response of construction employment in Panel (c). As with total employment, there is a sharp decline in the month of the disaster, followed by a recovery period with local construction employment peaking about a year out, when construction employment is estimated to be roughly $1.1 \%$ higher than in the no-disaster counterfactual. This is about six times larger than the total employment response at that horizon. The IRF of construction employment beyond one year flattens out somewhat but then, unlike total employment, steadily rises over the medium to longer run. As of eight years out, construction employment is estimated to be nearly $3 \%$ higher than it would have been in absence of the

\footnotetext{
${ }^{26}$ When only total damages are known for a given disaster, SHELDUS splits the total among all disasterdeclared counties equally. Given that more populous counties are likely to have more property at risk of damage, we adjust the data as follows. When we observe identical damages across counties for a given disaster, we redistribute total damages for that disaster to equate the per capita damages across affected counties.
} 
disaster. This suggests that the process of repairing and rebuilding public and private structures is, on average, quite long-lasting.

Panel (d) shows the quarterly IRF for average weekly wages (AWW) of local workers. AWW rise steadily after a disaster; by the end of the 8-year horizon, we estimate that AWW are about $0.5 \%$ higher than they would have been in absence of the disaster. Because AWW reflect the product of weekly hours and the hourly wage, this rise could be driven by an increase in hours worked per week, the hourly wage, or a combination of the two. ${ }^{27}$

Panel (e) displays the estimated IRF for quarterly house prices, based on the CoreLogic repeat-sales house price index (HPI). We find that the HPI is essentially flat in the near-term before increasing a good amount over the longer term. As of eight years after a disaster, the local HPI is estimated to be about $1.4 \%$ higher than it would have been otherwise. The lack of an increase in house prices in the first four to five years after the disaster suggests a limited role for damage-induced reductions in supply driving up prices. Rather, the longer run positive price effect appears to be in line with a steady or increasing demand for housing, which would in turn be consistent with the positive wage and income responses we observe. The longer-run increase in housing demand could push up house prices through either increased land valuations or improvements in the quality of the pre-existing housing stock. Improvements may be made in the process of rebuilding or repairing damaged homes or in the composition of houses being resold for the CoreLogic repeat sales index. That is, it is possible that homes are being rebuilt in more resilient locations (perhaps through managed retreat) or using better methods and materials, reflecting a shift in quality that could show up as an increase in the repeat-sales index.

We show the results for population in panel (f). We find that, on average, the response of population to a disaster is small and generally statistically insignificant up to at least eight years out. ${ }^{28}$ Thus we find that the average response is neither one of significant net out-

\footnotetext{
${ }^{27} \mathrm{We}$ also have examined whether the increase in average weekly wages could reflect a shift in employment composition toward higher-wage industries. As described in Online Appendix B2, we construct a measure of composition-adjusted wages using CEPR yearly extracts of the CPS Outgoing Rotation Group micro-data and Eckert, Fort, Schott, and Yang (2021)'s version of the Census Bureau's County Business Patterns (CBP) data. The estimated disaster IRF for composition-adjusted wages are shown in Appendix Figure A7. We find disasters lead to a shift in the composition of the local workforce toward higher income individuals, explaining at least part of the longer-run increase in average weekly wages.

${ }^{28}$ As shown in Appendix Figure A4, we obtain a somewhat different result if we use an alternative specification that replaces the county-specific pre-disaster linear time trend variable with a county-specific fullsample linear time trend variable (i.e., an interaction between the county fixed effect and year). As mentioned
} 
migration (in response to the negative effects of disasters) or significant net in-migration (in response to recovery efforts). However, these averages mask modest longer-run declines in both in-migration and out-migration that roughly cancel each other out (see Appendix Figure A8). ${ }^{29}$ Thus the near-zero population response is not due to a lack of migration responses. Taken together, the positive response of house prices and the relative non-response of population over the longer-run is consistent with a standard spatial equilibrium model with the average county having a relatively inelastic housing supply. A low elasticity of housing supply constrains population growth and stimulates house prices in response to a positive shock to local amenities and/or local productivity. Below we further explore this hypothesis using data on local housing supply elasticities.

Another component that could potentially explain our finding of higher personal income p.c. is government transfers. In Appendix Figure A9, we examine the impact of disasters on government transfers, including disaster aid and loans. Although federal disaster aid and insurance payouts increase substantially after a natural disaster as one would expect, we find that over the longer run overall government transfers are lower than they would have been in the absence of a disaster. ${ }^{30}$ This latter phenomenon appears to be driven by lower transfers for "income maintenance," as unemployment insurance transfers are essentially unchanged over the longer run. The lack of any longer run increase in these safety-net transfers is consistent with our findings for key components in Figure 3. In particular, one might expect fewer local households to qualify for safety-net programs over the longer run given that total employment is unchanged while average weekly wages are higher.

\section{Economic Mechanisms}

Our baseline results point to a longer-run increase in local personal income after a natural disaster. This increase owes to higher labor income - which appears to stem from a longer-run increase in average weekly wages - and not government transfers, which actually decline over

earlier, our preferred specification does not include the latter because it is potentially endogenous with respect to the disaster treatment. Nonetheless, using that specification yields results that are broadly similar to the baseline results for all outcomes except population. This specification yields an IRF for population that is steadily declining over time. As of eight years out, population is estimated to be a little over $-0.1 \%$ below the no-disaster counterfactual.

${ }^{29}$ Appendix Figure $\mathbf{A 8}$ shows results from estimating the IRFs separately for in-migration and out-migration, where each outcome is measured as the number of migrants divided by pre-disaster $(t-1)$ population. See

Appendix B5 for details on the migration data.

${ }^{30}$ In results not shown, we find that aid increases with disaster severity. 
the longer-run. ${ }^{31}$ The increase in earnings is consistent with a long-lasting process of recovery and rebuilding — as reflected by the longer-run increase in construction employment - along with, potentially, productivity gains from improved local public and private capital stock. The hypothesis that the local capital stock is substantially improved is supported by our finding of higher house prices over the longer run. ${ }^{32}$

These results are consistent with spatial equilibrium models to the extent that disasters result eventually in improvements to local capital, which in turn improve local productivity and amenities. In such models, increases in productivity and amenities result in higher demand for both labor and housing. Hornbeck and Keniston (2017) suggest a specific channel for how disasters might lead to improvements in local public and private capital, pushing up local land values. In their model, property owners do not internalize the fact that improvements to their own properties have a positive externality on the values of other properties in the local area. This leads to a suboptimal level of property quality. A disaster compels and through insurance and aid provides funds for many local property owners to simultaneously rebuild or renovate properties up to current codes and standards, which raises the value of all local properties, even those not renovated.

A prediction of their model is that this channel is only operative in areas in which property values were growing; in declining areas, owners have no incentive to invest in their properties regardless of the externality. We thus test this prediction in our sample by estimating the house price disaster IRF separately for growing vs. declining counties, based on a county's 3-year predisaster growth rate of the house price index (deflated by the CPI). The results are shown in Figure 4. Consistent with the Hornbeck and Keniston (2017) model, we find that the positive response of house prices to a disaster shock applies only to growing counties.

As mentioned earlier, the fact that we see longer-run increases in house prices, but not in population, also suggests that housing supply is relatively inelastic on average, at least within our eight-year horizon. Indeed, two predictions of standard spatial equilibrium models, such as that of Hsieh and Moretti (2019), are (i) that house prices should respond more to disasters in areas with relatively inelastic housing supply, while (ii) population should respond more to disasters in

\footnotetext{
${ }^{31}$ See Appendix Figure A10 for IRF estimates for the wage and salary component of personal income.

${ }^{32}$ Note that if the increase in the house price index reflects an increase in the quality-adjusted cost of housing services, then it implies an increase in local consumer prices (cost of living), which could partially offset the benefits of higher income for local residents.
} 
areas with relatively elastic housing supply. We test these predictions by estimating the house price and population disaster IRFs separately for counties with a Saiz (2010) housing supply elasticity above the median ("elastic") vs. below the median ("inelastic"). The results are shown in Figure 5. We find strong evidence supporting both predictions. Namely, the increase in house prices after a disaster occurs only in local areas where housing supply is relatively inelastic, while a post-disaster increase in population occurs only in areas where housing supply is relatively elastic. The intuition is that places where housing supply can easily expand in response to higher demand (driven by higher amenities and/or higher productivity) can accommodate a higher population, whereas in places where housing supply is closer to fixed, the higher demand drives up prices.

Similarly, that average weekly wages increase over the longer-run while employment returns to pre-disaster levels, might suggest that external labor supply - that is, net in-migration of workers - is relatively inelastic on average. However, results in Appendix Figure A9 on composition-adjusted wages point to an alternative interpretation. ${ }^{33}$ There we find natural disasters likely lead to a shift in the composition of the local workforce toward higher income individuals, explaining at least part of the longer-run increase in (unadjusted) average weekly wages. ${ }^{34}$ As such, quality/composition-adjusted employment could be said to have increased while composition-adjusted wages were closer to flat, consistent with a relatively elastic labor supply margin via migration.

\section{Heterogeneity, Adaptation, and Spatial Spillovers}

We now explore three sets of extensions relating to heterogeneity of disaster responses, adaptation to disasters over time and based on county-specific prior experience, and spatial spillover effects.

\section{A. Heterogeneity}

Although informative for many policy questions, the average dynamic response of local economic activity to natural disasters presented above may mask heterogeneity along several

\footnotetext{
${ }^{33}$ See Appendix B2 for a discussion of how we construct a measure of composition-adjusted wages using CEPR yearly extracts of the CPS Outgoing Rotation Group micro-data and Eckert, Fort, Schott, and Yang (2021)'s version of the Census Bureau's County Business Patterns (CBP) data.

${ }^{34} \mathrm{We}$ assume here that changes in industry mix affect average weekly wages (AWW) by affecting average hourly wages. It is also possible that changes in industry mix affect AWW by affecting average hours per week if hours per week vary by industry, in which case our results here understate the role of industry mix.
} 
important dimensions. Understanding how the economic impacts differ across different kinds of disasters and different economic pre-conditions can also be important. In this section, we consider heterogeneity in terms of disaster severity, disaster type (i.e., floods, hurricanes, etc.), and county income prior to the disaster.

\section{Heterogeneity by disaster severity}

In our first heterogeneity analysis, we examine disaster severity, which is important in light of projections that some disasters will become more severe with climate change (USGCRP 2017). We seek to understand both whether the estimated average effects apply to the most severe disasters and whether the most severe disasters are driving the estimated average effects. To explore how the economic response to a disaster varies with damages, we estimate the impulse response at horizon $h$ to a third-order polynomial function of direct disaster damages:

$$
\beta^{h}(s)=\sum_{p=0}^{3} \beta_{p}^{h} s_{c, t}^{p}
$$

where $s_{c, t}$ denotes the per capita damages for county $c$ in period $t$. Substituting equation (3) into equation (1) yields the following specification:

$$
\begin{aligned}
y_{c, t+h}-y_{c, t-1}= & \beta_{0}^{h} D_{c, t}+\beta_{1}^{h} D_{c, t} s_{c, t}+\beta_{2}^{h} D_{c, t} s_{c, t}^{2}+\beta_{3}^{h} D_{c, t} s_{c, t}^{3} \\
& +\boldsymbol{X}_{c t}^{\prime} \gamma^{h}+\alpha_{r(c), t}^{h}+\alpha_{c, m(t)}^{h}+\epsilon_{c, t+h}
\end{aligned}
$$

The estimated coefficients, $\hat{\beta}_{p}^{h}$ for $p=0, \ldots, 3$, from this regression allow us to compute the impulse response, $\hat{\beta}^{h}(s)$, for any given level of damages (s) according to equation (3).

We use SHELDUS data to measure damages for our main disaster heterogeneity analyses. As described in more detail section VI.B., these data are based on the NOAA Storm Database. We use damages aggregated at the county-month level, which we translate to a per capita basis using Census population data. ${ }^{35}$ A potential concern with measuring disaster severity using monetary damages is endogeneity. An alternative approach found in some prior studies is to use data on physical disaster characteristics, such as wind speed for

\footnotetext{
${ }^{35}$ As described in section VI.B., we adjust the SHELDUS data to redistribute duplicate observations to be equal across counties at a per capita level. Our main personal income result here is robust to using the original unadjusted data.
} 
hurricanes to estimate responses to different treatment intensities. ${ }^{36} \mathrm{We}$ focus here on pecuniary damage data because historical data on physical characteristics are not readily available at the U.S. county level for a broad set of disaster types and are not comparable across disaster types when available. In addition, we are inherently interested in natural hazards that result in disastrous outcomes, which are a function of the built environment that those hazards occur in. The monetary damages caused by a physical event depend greatly on the quantity and market value of local property as well as building codes, construction quality, infrastructure, and other factors that affect resilience. ${ }^{37}$ Nonetheless, given potential concerns with measuring severity using damages and to facilitate comparisons to prior studies of hurricanes, we also present results below specifically for hurricanes using wind speed to measure disaster severity.

For the same six economic outcomes as in Figure 3, Figure 6 displays the estimated IRFs for different levels of damages corresponding to various percentiles of the distribution of (nonzero) damages p.c. in our sample. In each panel the solid thick blue line depicts the IRF corresponding to a disaster with per capita damages equal to the $50^{\text {th }}$ percentile of all disasters (with positive damages), while the thick solid orange line depicts the IRF for a disaster with per capita damages equal to the $99^{\text {th }}$ percentile. The thin solid, dashed, and dash-dotted lines show the IRFs for other percentile damages.

Panel (a) shows the results for personal income p.c. The IRFs for $25^{\text {th }}-75^{\text {th }}$ percentile damages disasters are quite similar to the baseline IRF shown in Figure 3, panel (a), with a modest initial drop followed by a modest positive response over the medium run and a larger positive response over the longer run. Notable differences in either the level or shape of the IRF do not really emerge until damages rise above the $95^{\text {th }}$ percentile. For the most severe disastersthose with damages above the $95^{\text {th }}$ percentile - personal income p.c. increases substantially both in the short-run and over the longer-run. For instance, we estimate that a $99^{\text {th }}$ percentile disaster causes personal income p.c. to increase by nearly $2.5 \%$ in the initial year and over the longer run.

\footnotetext{
${ }^{36}$ For example, Deryugina (2017) used FEMA's HAZUS-MH simulation model to estimate damages for major hurricanes in the U.S. as a function of wind speed and other storm characteristics. Hsiang and Jina (2014) use a reduced form approach to estimate the economic impact of major hurricanes around the world as a function of the wind speeds associated with each hurricane. Felbermayr and Groschl (2014) expand that approach to cover other types of disasters, using international geophysical and meteorological data. Similarly, Lackner (2019) estimates the impact of earthquakes, measuring their severity using spatially disaggregated data on ground shaking.

${ }^{37}$ For example, Bakkensen and Mendelsohn (2016) show that hurricane damages tend to be higher in the U.S. than in other OECD countries when examining responses to physical storm characteristics.
} 
These results indicate that, perhaps counterintuitively, the positive response of income per capita to disasters increases with the severity of the disaster.

Looking at the analogous results for the other outcomes in panels (b)-(f) allows us to unpack this result. First, we find that the most severe disasters cause large and persistent increases in both total employment (panel b) and average weekly wages (panel d), with employment and AWW up over $1 \%$ after eight years. ${ }^{38}$ Construction employment increases by about $6 \%$ within the first year after the disaster but after a few years comes down to a level similar to that after a more typical disaster. In other words, the short- to medium-run increase in construction activity after a disaster is much higher for very severe disasters, but the longerrun increase is roughly independent of the disaster's severity.

We uncover an interesting pattern for house prices in panel (e), in that the medium-run (1-3 years out) response to very severe disasters is strongly positive while the longer-run response is strongly negative. This pattern contrasts with the baseline, average-disaster results in Figure 3, panel (e), in which house prices are flat in the first few years and then rise over the longer run. This longer-run decline in house prices after very severe disasters may be partially explained by the population responses shown in panel (f). Population falls substantially over several years after the most severe disasters. ${ }^{39}$ This longer-run drop in population should reduce demand for housing, putting downward pressure on home prices. Taken together, the responses of house prices and population after severe disasters point to negative housing supply shocks — which push up prices despite population losses—playing a dominant role in the shorter run and negative housing demand shocks—-which push down both prices and population - playing a dominant role in the longer run.

In addition, the longer-run population response helps explain the magnitude of the longer-run increase in personal income p.c. In particular, we find that a disaster with damages p.c. equal to the $99^{\text {th }}$ percentile causes population to decline by roughly $1.5 \%$ after

${ }^{38}$ The large increase in average weekly wages over the longer run is consistent with the worker-level evidence of higher long term wages following the major 2005 hurricanes, Katrina and Rita, provided by Groen, Kutzbach, and Polivka (2020).

${ }^{39}$ Appendix $\mathbf{A 1 0}$ provides results separately for in-migration and out-migration. In-migration is measured as the total flow of in-migrants to the county from period $t$ to $t+h$ as a share of pre-disaster population. Out-migration is defined analogously. While both increase substantially in the first 3-4 years after the most severe disasters, the out-migration effect is much larger. Over the longer-run (up to 8 years out), both the in- and out-migration responses to severe disasters become somewhat negative, though out-migration continues to be higher (less negative). The higher out-migration response is consistent with the estimated decline in population over the medium and longer runs for the most severe disasters. These results points to considerable population "churn" after a disaster. 
eight years. This result in turn suggests that most (1.5 percentage points) of the roughly $2.5 \%$ longer-run increase in personal income p.c. after very severe disasters (panel (a)) is due to population loss while the remainder is due to higher aggregate personal income.

On the whole, these results suggest that the magnitude of our baseline responses increase with severity. Here we have measured severity with monetary damages per capita, which should reflect the magnitude of the destruction of public and private capital (including residential capital) in the immediate aftermath of the disaster. As we noted above, a concern might be that measured damages are endogenous, at least in the short run, in that they could pick up not only losses of capital/wealth but also initial income losses. If reductions in initial income lead to higher measured damages, any resulting bias for the estimated income impulse response function should be negative and primarily apply to our coefficient estimates for the initial horizons. Thus, to the extent this is a concern, the larger income per capita response we find for the most severe disasters should be considered a lower bound on the true role of severity, at least for the short-run response for which the endogeneity concern is most relevant.

To assess whether physical measures of disaster severity yield different results than monetary damages, we re-estimate equation (4) just for hurricane disasters, once using damages p.c. to measure severity, $s_{c, t}$, and once using maximum sustainable wind speed. ${ }^{40}$ In each case, the percentiles of the severity distribution are calculated over only hurricane disasters. The results are shown in Figure A11. Panel (a) shows that the IRF by damages for hurricanes is qualitatively similar to the IRF by damages for all disasters - namely that the income p.c. response is positive and larger for more damaging hurricanes at all horizons. Panel (b) shows the IRF by wind speed is positive and increases with wind speed in the short to medium run, but it is close to zero and decreases with wind speed in the longer run. Thus, damages and wind speed yield qualitatively similar results in the short to medium run, but different results at longer horizons. This difference is unlikely to be attributable to any endogeneity of damages given that any such endogeneity should primarily be a short-run phenomenon. The difference may stem from wind speed not fully capturing the true economic severity of hurricanes because it does not

\footnotetext{
${ }^{40}$ The wind speed data are described in Appendix B4.
} 
reflect damage from water or varying vulnerabilities of infrastructure. In other words, measurement error could be leading to attenuation bias toward zero. ${ }^{41}$

In sum, we find that increases in personal income are generally larger for more severe disasters. What can explain this phenomenon? One possibility is that very severe disasters trigger investment in new, modern public and private infrastructure, funded perhaps by government aid as well as private insurance, which spurs local economic development, consistent with the "build back better" scenario from Figure 2. This is not unlike the theory and evidence on war destruction of capital, and subsequent investment-led growth, discussed in Section III. Another possibility, consistent with the large decline in population, is that the composition of households in affected counties is changed by the most severe natural disasters, with lowerincome households more likely to move out of the county. ${ }^{42}$ This possibility is consistent with Sheldon and Zhan (2021), who find that post-disaster out-migration increases with the severity of the disaster and more so the lower the income of the population. ${ }^{43}$

\section{Heterogeneity by type of disaster}

We next explore how economic responses to natural disasters vary by disaster type. Specifically, we estimate the following joint regression:

$$
y_{c, t+h}-y_{c, t-1}=\sum_{d \in \mathcal{D}} \beta_{d}^{h} D_{c, t}^{d}+\boldsymbol{X}_{c t}^{\prime} \gamma^{h}+\alpha_{r(c), t}^{h}+\alpha_{c, m(t)}^{h}+\epsilon_{c, t+h}
$$

where $D_{c, t}^{d}$ is an indicator for a disaster of type $d$. The set of disaster types, $\mathcal{D}$, consists of hurricanes, floods, severe storms, extreme winter weather, fires, tornados, and other. The estimates of $\beta_{d}^{h}$ trace out the IRF of the outcome variable with respect to a disaster of type $d$. For

\footnotetext{
${ }^{41}$ For example, wind speed may not reflect the flooding damage associated with hurricanes, which could have long-term effects on income per capita. In addition, high wind speed hurricanes could have a very different longer run effect in a county with significant property value at risk than in a county with little value at risk or in a county with significant investments in resilient infrastructure than one without.

42 Indeed, as shown in Appendix Figure A8, we find that after the most severe damages both in-migration and out-migration increase over the medium term, with out-migration apparently dominating such that population falls. In the longer-run, in-migration declines more than out-migration for the most severe disasters.

${ }^{43}$ It is worth noting that our finding of a significant longer-run increase in income p.c. after very severe disasters is consistent with the observed pattern of income p.c. following the most severe disaster in our sample, Hurricane Katrina in 2005. Appendix Figure A12 plots actual income p.c. for Orleans Parish, Louisiana, from 1980 to 2017. Relative to the approximately linear trend up to 2005 , income p.c. spikes in the first $2-3$ years after the disaster before gradually returning to the pre-disaster growth trend but at a permanently higher level.
} 
these regressions, we modify the first term of the control vector so that the leads and lags of disasters are differentiated by type: ${ }^{44}$

$$
\boldsymbol{X}_{c t}^{\prime} \boldsymbol{\gamma}^{\boldsymbol{h}} \equiv \sum_{d \in \mathcal{D}} \sum_{\substack{\tau=-p, \tau \neq 0}}^{h} D_{c, t+\tau}^{d}+\sum_{\tau=-p}^{h} \theta^{\tau h} D_{c, t+\tau}^{0}+\rho^{h} \Delta y_{c, t-1, t-k}
$$

The results are shown in Figure 7. We find significant heterogeneity in how personal income p.c. responds to different types of natural disasters. There are substantial medium- to longer-run increases in personal income p.c. for hurricanes, tornados, and fires. However, fires are quite rare in our sample, accounting for just 2 percent of the disasters, and thus their IRFs are imprecisely estimated. Non-hurricane floods, on the other hand, account for $60 \%$ of the disasters in our sample. For these floods, we estimate a statistically significant negative effect in the year of the disaster, followed by a modest positive effect in the medium term, and no significant effect in the longer run.

There are several potential explanations for this finding. First, it could reflect differences in destruction. As shown in Appendix Figure A13, disaster types differ significantly in terms of estimated damages, with hurricanes and tornadoes tending to have the highest per capita damages in affected counties. To the extent that hurricanes and tornados tend to more fully destroy structures such that they must be completely rebuilt, this could yield more productivityenhancing improvements in the capital stock. Second, floods may disproportionately increase the perceived risk of future flooding in the same exact location, reducing incentives to make rebuilding and recovery investments. ${ }^{45}$ In contrast, hurricanes and tornados may be perceived to be unlikely to strike the same exact spot again soon. Third, wind damage is generally covered by standard homeowner insurance plans while flood damage is not. Greater insurance coverage of damages from hurricanes and tornados than floods could lead to larger gains from recovery efforts.

Interestingly, our results on hurricanes here contrast somewhat with prior findings by Strobl (2011). Strobl finds that income p.c. growth falls significantly in coastal U.S. counties in the

\footnotetext{
${ }^{44}$ For monthly outcomes, due to computational demands, we control for 12-month aggregate indicators for the leads and lags of each disaster type.

${ }^{45}$ Giglio, Maggiori, Rao, Stroebel, and Weber (2021) find that increases in perceived risk of future flooding in a local area (ZIP code) are associated with declines in local real estate prices, which would reduce the incentive to invest in new real estate construction projects.
} 
initial year of a hurricane and then returns to the pre-hurricane growth rate in the following year. This dynamic growth pattern translates into an initial year decline in the level of income p.c. that is not made up thereafter (which would require above-trend growth in the following year). That pattern contrasts with our finding of a positive impact even in the first year after a disaster. Strobl's estimates do not speak to whether income rises or falls beyond one year out.

\section{Heterogeneity by county pre-disaster income}

In our third heterogeneity analysis, we examine the role of pre-disaster income in the disaster response. This analysis is prompted by the findings in the literature that lower income countries see worse outcomes in GDP after natural disasters. Furthermore, households in higher income counties may have more private insurance. This, combined with reports that lower income households tend to receive less aid, would lead to higher income counties having more resources with which to fund their recovery and rebuilding activities.

To investigate heterogeneity in treatment effects in terms of initial income, we split county*year observations into four groups based on their quartile of the distribution of prioryear $(t-1)$ personal income per capita. We then interact the disaster indicator with the income quartile variable, estimating the following specification:

$$
\begin{aligned}
y_{c, t+h}-y_{c, t-1} & =\sum_{q=1}^{4} \beta^{h, q} M_{c, t-1}^{q} D_{c, t}+\sum_{q=1}^{4} \phi^{h, q} M_{c, t-1}^{q}+\boldsymbol{X}_{c t}^{\prime} \boldsymbol{\gamma}^{\boldsymbol{h}} \\
& +\alpha_{r(c), t}^{h}+\alpha_{c, m(t)}^{h}+\epsilon_{c, t+h}
\end{aligned}
$$

where $M_{c, t-1}^{q}$ is one of four income quartile indicators indexed by $q$ and $\boldsymbol{X}_{c t}^{\prime} \gamma^{\boldsymbol{h}}$ is as defined earlier in equation (2). The $\beta^{h, q}$ coefficients trace out a separate impulse response function for each quartile $q$. We also include the four quartile indicators themselves as separate conditioning variables in the regression to ensure against possible "selection into treatment" - that is, the possibility that either higher or lower income counties are more likely to be hit by a disaster.

In Figure 8 we show the results of estimating equation (7), which estimates separate IRFs by quartile of pre-disaster county income. We find that the point estimates for all four quartiles are consistent with our baseline results of a longer-run (as of eight years out) increase in income p.c., although they are not all statistically significant. Furthermore, the timing of the increase differs, as only counties in the top quartile of pre-disaster income p.c., experience a statistically 
significant increase in income in the first four years after the disaster (relative to the no-disaster counterfactual.) Thus, if we looked only a couple of years post-disaster, our findings would be consistent with those showing increases in income or GDP only for higher income countries.

\section{B. Adaptation}

An increase in disaster frequency can spur adaptative investments in resilience, as greater experience with disasters leads to learning and expectations of more future disasters. However, intensification can also overwhelm disaster response and recovery capacity, making it more difficult to rebuild quickly and effectively. Each of these phenomena could shift average responses to disasters as they become more frequent over time. We take two approaches to examine these issues.

First, we investigate whether disaster IRFs vary with a county's historical experience with disasters. To do so, we estimate equation (7), where $M_{c, t-1}^{q}$ for each $q \in Q$ is an indicator for whether the county experienced (a) no periods, (b) 1 period, (c) 2-3 periods, or (d) 4 or more periods with disasters in the previous 10 years. ${ }^{46} \mathrm{We}$ again interact the category indicators with the disaster indicator (as well as including the category indicators as separate regressors).

The results in Figure 9, panel (a) show limited evidence of adaptation. In contrast to the notion that disaster-prone areas adapt and become more resilient to disasters, we find that only counties with significant disaster experience show an immediate negative hit to income p.c. (statistically significant at the $90 \%$ level for the top category). This suggests a possible role for intensification, whereby counties hit by other disasters within the previous 10 years may not have had time to fully recover and rebuild private and public capital, leaving them vulnerable to the impact of new disasters. In the medium- to longer-run, increased personal income p.c. occurs in all cases but is least significant for counties that have experienced the most disasters in the prior ten years, again suggesting a role for intensification rather than adaptation.

Our second analysis addresses a closely related adaptation question on how the average disaster response across all counties — regardless of their individual disaster experiences - has evolved over time. Given the increased aggregate frequency of disasters that we documented in Figure 1, communities throughout the United States may be anticipating a higher likelihood of disasters going forward, which could in turn lead to adaptations to mitigate the initial negative

\footnotetext{
${ }^{46}$ Periods are monthly, quarterly, or annual, based on the outcome of interest.
} 
impacts on income, not to mention the negative impacts to wealth, property, and health. Furthermore, a more resilient infrastructure could yield smaller boosts to income that result from rebuilding and recovery efforts.

We interact our disaster indicator with a continuous time variable in order to estimate how the impulse response function has evolved over time as shown below:

$$
y_{c, t+h}-y_{c, t-1}=\theta^{h} \cdot t \cdot D_{c, t}+\beta^{h} D_{c, t}+X_{c t}^{\prime} \gamma^{h}+\alpha_{r(c), t}^{h}+\alpha_{c, m(t)}^{h}+\epsilon_{c, t+h} .
$$

Here $\theta^{h}$ estimates how the response has evolved over time, where a positive value would reflect that the marginal effect of a disaster on personal income has increased over time. To illustrate the results, we then calculate the implied IRF for the earliest (1983) and latest (2009) years in the sample, excluding the years in the earliest pre-trend interval (3 years) and the latest longer-run interval ( 8 years) using our estimate of $\widehat{\theta^{h}}$.

The results for income p.c. in Figure 9, panel (b) show that the initial impact coefficient has become less negative, shifting toward zero over time, consistent with adaptation; however, the shift is not statistically significant. The magnitude of the longer-run postdisaster increase in income has also decreased over time, but not by much. The notable difference is in the medium run. For the earliest year of the analysis (1983), we find a significant boost in personal income started in the third year after the disaster and peaked in in the fourth year. In contrast, as of the latest year in our analysis (2009) the estimated medium-run effect was negative for the fourth and fifth years after the disaster. This could be explained by disaster recovery resources becoming increasingly strained as the frequency of disasters has risen, thereby slowing down the p.c. personal income recovery over the decades. Going forward, as disasters become more frequent and severe, these results suggest that counties hit by disasters may see slower and smaller boosts in personal income.

\section{Spatial Spillovers and Wider Geographies}

We have thus far focused on estimating the economic impacts of natural disasters at the local level, proxied by counties. The impacts at higher levels of aggregation could be different as important spatial spillovers could propagate the effects of a disaster in one county to other counties of varying distances away. We now investigate this issue in two complementary ways. First, we construct geographic circular bands ("donuts") of counties of given distance ranges surrounding each focal county hit by a disaster and then jointly estimate the impact of disasters 
on both the focal county and on the geographic areas defined by each surrounding band. Second, we aggregate to the state level our treatment and outcome variables and repeat our baseline IRF specifications at the state, rather than county, level.

For our first approach, we build on our baseline specification in equation (1) by adding continuous treatment variables $D_{c, t}^{b}$ measuring the occurrence of disasters in other counties of varying distances away from county $c$ :

$$
y_{c, t+h}-y_{c, t-1}=\sum_{b \in B} \pi^{h, b} D_{c, t}^{b}+\beta^{h} D_{c, t}+X_{c t}^{\prime} \gamma^{h}+\alpha_{r(c), t}^{h}+\alpha_{c, m(t)}^{h}+\epsilon_{c, t+h}
$$

For any given focal county, $c$, we split all other counties into $B$ separate distance bands (“donuts") indexed by $b$.

The treatment variable $D_{c, t}^{b}$ is then defined as the share of population within distance band $b$ from county $c$ that was in counties that experienced a disaster in period $t$ :

$$
D_{c t}^{b} \equiv \sum_{i \neq c} 1\left[b \leq d_{c i}<b^{\prime}\right] \omega_{c i} D_{i t}
$$

where

$$
\omega_{c i} \equiv \frac{\operatorname{pop}_{i}}{\sum_{i} 1\left[b \leq d_{c i}<b^{\prime}\right] p o p_{i}},
$$

and $p o p_{i}$ denotes population of county $i$ and $d_{c i}$ denotes the distance between the population centroids of counties $c$ and $i$. For example, if county $c$ has 10 million people living within 200399 miles of it, and there is a disaster in year $t$ in a county or counties in that band covering a population of 2 million, then $D_{c, t}^{200}$ would be 0.2 .

In Figure 10, we show the results of estimating equation (9) for personal income for bands of counties that are up to 199, 200-399, and 400-599 miles away from a county affected by a disaster. ${ }^{47}$ The thin blue curves show the IRFs for the directly-hit counties, while the orange curves show the spatial lag IRFs. ${ }^{48,49}$ These results show that nearby counties (within 199 miles)

\footnotetext{
47 See Appendix Figure A14 for a visual illustration of the spatial lags for a single year of disasters.

${ }^{48}$ Note that the blue curve shown here differs from our baseline estimate shown in Figure $\mathbf{3}$ because many disasters affect multiple neighboring counties, in which case we would have to add to the blue curve the effect captured in the orange curve in panel (a).

${ }^{49}$ In panels (a)-(c), the spatial lag coefficients have been normalized such that $D_{c, t}^{b}$ has been divided it by its mean, conditional on $D_{c, t}^{b}>0$. Given this normalization, a one-unit change in each spatial lag variable represents the average population share in that distance band of disaster-hit counties in the event of at least one disaster. These conditional means vary slightly across horizons because the regression samples are horizon-specific. For the 50-199
} 
experience a medium-run boost to personal income, consistent with residents of nearby counties participating in recovery efforts and experiencing positive spillovers (panel (a)). However, these counties do not appear to share in any longer run boost to income per capita. Counties that are 200-399 miles away see a persistent decline in personal income, as shown in panel (b). This could be explained by regional resources being redirected to the counties directly affected by disasters. Counties in the furthest band, 400-599 miles away, may experience some modest intermediate gains in income per capita followed by a longer run decline, but these findings are generally not statistically significant (panel (c)).

In panel (d) we show an estimate for the net effect on personal income per capita within 600 miles of disasters. Here we estimate the sum of the four curves shown in panels (a) - (c), where each IRF is rescaled by multiplying each of the $\hat{\beta}^{h}$ and $\hat{\pi}^{h, b}$ terms by the unconditional mean of the corresponding variable. With the estimated $\hat{\beta}^{h}$ and $\hat{\pi}^{h, b}$ coefficients representing the average effect for a county within each category at each horizon, intuitively, we are taking an average of the contribution of these responses to estimate a net effect. This post-estimation rescaling of the IRF coefficients is equivalent to a pre-estimation mean-normalization. These results suggest that while the longer run local impact of a disaster on income per capita in the area directly hit by a disaster is positive, the longer run impact for the broader region appears to be negative.

Turning to our second analysis, we estimate the equation (1) baseline specification at an aggregated state level by replacing the $D_{c, t}$ indicator with a continuous measure of the share of the state's population living in counties hit by disasters in period $t .{ }^{50}$ For this analysis, we cluster the standard errors at the state and time-by-Census Division levels.

The results in Figure 11 indicate that the dynamic pattern for this state-level analysis is qualitatively similar to our baseline per capita income result at the county level (Figure 3, panel (a)). However, the state-level disaster impacts are small and insignificant at the 95\% level, consistent with the Figure 10 results showing a statistically insignificant net effect of disasters on income per capita for broad geographic areas. ${ }^{51}$

mile band, the conditional mean varies from 0.27 to 0.28 . For the $200-399$ band, it rounds to 0.24 across all horizons; and for the 400-599 band, it varies from 0.23 to 0.24 . The coefficients $\pi^{h, b}$ can then be interpreted as the impact in county $c$ from the average disaster event hitting at least one county $b$ to $b$ ' miles away.

${ }^{50}$ Using a simple indicator variable for whether there was a disaster in any county in a given state-year results in very few no-treatment observations and also lumps together disasters of widely varying geographic breadth.

${ }^{51}$ We have also investigated whether the state-level impact varied with the scale/breadth of the disaster by interacting the disaster treatment variable with four dummy variables indicating whether the percentage of the state 


\section{Conclusion}

We have found that, on average, counties hit by natural disasters experience a medium- to longer- run boost in income per capita. While a rise in employment contributes to the initial boost, the longer-run increase in income per capita is driven largely by an increase in average weekly wages. House prices are also found to increase over the longer run while population is roughly unchanged.

These results can be explained in the context of standard spatial equilibrium models whereby disasters result eventually in improvements to local capital, which in turn improve local productivity and amenities. Increases in productivity and amenities increase demand for both labor and housing. Our findings that disaster leads to longer-run increases in wages and house prices, rather than employment and population, suggest relatively inelastic supply of both labor and housing on average. Indeed, we are able to directly examine the role of the supply elasticity for housing by estimating disasters' effects on house prices and population separately for high and low housing supply elasticity counties using Saiz's (2010) elasticity estimates. Consistent with theory, we find the post-disaster increase in house prices occurs only in counties where housing supply is relatively inelastic, while an increase in population after a disaster occurs only in areas where housing supply is relatively elastic.

This theory can also help explain some of the heterogeneity we observe. In particular, we observe increases in personal income after hurricanes and tornadoes but not after floods. If the perceived probability of repeat incidents in a location is high after floods but low after hurricanes and tornadoes, the willingness to make the recovery investments that improve local capital stock may be much greater after hurricanes and tornadoes than floods. Indeed, under the Hazard Mitigation Grant Program, FEMA has bought out tens of thousands of flood-prone homes in recent decades to mitigate National Flood Insurance Costs through managed retreat (Kousky 2014, Mach, et al. 2019).

In addition, while the main focus of this paper has been on the local impact of natural disasters, our spatial analyses suggests that the long run increase in personal income locally may come at the cost of, and be more than offset by, a long run decline in personal income in

population in a county hit by a disaster was below $10 \%, 10-50 \%, 50-90 \%$, or above $90 \%$. Shown in panel (b) of Appendix Figure A15, the longer-run positive impacts are found to increase with the scale/breath of the disaster, though the results are not statistically significant. 
surrounding counties. This could potentially be explained by a diversion of resources to areas affected by disasters.

Taken together, our results suggest that despite the immense toll that disasters take, local economies in the U.S. have generally recovered successfully in terms of income. Indeed, not only does local income recover fully after a few years, it ends up somewhat higher in the longer run than what it would have been without the disaster. However, this average finding masks significant heterogeneity across contexts and a potential rise in inequality after disasters, given the lack of any decline in the poverty rate.

Our results contrast with some prior international evidence, especially research focused on developing countries, that has found long-lasting negative effects of certain disasters on national income. An important difference between developing countries and the U.S. is the wide availability in the U.S. of private and public insurance, the latter coming in the form of government aid. Going forward, climate change may lead to more frequent and more damaging disasters, which could put increasing demands on insurance markets, government budgets, and the construction sector. 


\section{References}

Angrist, Joshua D. and Jörn-Steffen Pischke (2009). "Mostly Harmless Econometrics : An Empiricist's Companion," Princeton University Press, Princeton.

Bakkensen, Laura A. and Lint Barrage (2020). "Climate Shocks, Cyclones, and Economic Growth: Bridging the Micro-Macro Gap.” No w24893. National Bureau of Economic Research.

Bakkensen, Laura A. and Robert O. Mendelsohn (2016). "Risk and Adaptation: Evidence from Global Hurricane Damages and Fatalities," Journal of the Association of Environmental and Resource Economists, University of Chicago Press, vol. 3(3), pages 555-587.

Barattieri, Alessandro, Patrice Borda, Alberto Brugnoli, Martino Pelli, and Jeanne Tschopp (2023). "The short-run, dynamic employment effects of natural disasters: New insights from Puerto Rico," Ecological Economics, 205 (107693).

Belasen, Ariel R. and Solomon W. Polachek (2008). "How Hurricanes Affect Wages and Employment in Local Labor Markets," American Economic Review: Papers \& Proceedings, 98 (2): 49-53.

Bin, Okmyungm and Craig E. Landry (2013). Changes in implicit flood risk premiums:

Empirical evidence from the housing market, 65, no. 4: 361-376.

Botzen, W J Wouter, Olivier Deschenes, Mark Sanders (2019). “The Economic Impacts of Natural Disasters: A Review of Models and Empirical Studies," Review of Environmental Economics and Policy, rez004.

Boustan, Leah Platt, Matthew E. Kahn, Paul W. Rhode, and Maria Lucia Yanguas (2020). "The Effect of Natural Disasters on Economic Activity in U.S. Counties: A Century of Data," Journal of Urban Economics, 118 (103257).

Bureau of Economic Analysis (BEA) (2017). "Local Area Personal Income and Employment Methodology."

Cavallo, Eduardo, Sebastian Galiani, Ilan Noy, and Juan Pantano (2013). "Catastrophic natural disasters and economic growth." Review of Economics and Statistics, 95(5): 1549-1561. Chodorow-Reich, Gabriel (2019). “Geographic Cross-Sectional Fiscal Spending Multipliers:

What Have We Learned." American Economic Journal: Economic Policy, 11(2): 1-34 Chodorow-Reich, Gabriel, Laura Feiveson, Zachary Liscow, and William Gui Woolston (2012).

"Does state fiscal relief during recessions increase employment? Evidence from the 
American Recovery and Reinvestment Act." American Economic Journal: Economic Policy 4, no. 3: 118-45.

Council of Economic Advisors (CEA)(2022). "The Rising Costs of Extreme Weather Events." available at https://www.whitehouse.gov/cea/written-materials/2022/09/01/the-rising-costsof-extreme-weather-events/

Davis, Donald R., and David E. Weinstein (2002). "Bones, bombs, and break points: the geography of economic activity." American Economic Review 92, no. 5: 1269-1289.

Deryugina, Tatyana (2017). "The fiscal cost of hurricanes: disaster aid versus social insurance." American Economic Journal: Economic Policy 9.3: 168-98.

Deryugina, T., Kawano, L., \& Levitt, S (2018). “The economic impact of hurricane Katrina on its victims: evidence from individual tax returns." American Economic Journal: Applied Economics: 202-33.

Eckert, Fabian, Teresa C. Fort, Peter K. Schott, and Natalie J. Yang. "Imputing Missing Values in the US Census Bureau's County Business Patterns." NBER Working Paper \#26632, 2021.

Felbermayr, G., \& Gröschl, J. (2014). "Naturally negative: The growth effects of natural disasters." Journal of development economics, 111, 92-106.

Gallagher, Justin, and Daniel Hartley (2017). "Household finance after a natural disaster: The case of Hurricane Katrina." American Economic Journal: Economic Policy 9, no. 3: 199-228.

Giglio, Stefano, Matteo Maggiori, Krishna Rao, Johannes Stroebel, and Andreas Weber. "Climate change and long-run discount rates: Evidence from real estate." The Review of Financial Studies 34, no. 8 (2021): 3527-3571.

Goodman-Bacon, Andrew. "Difference-in-differences with variation in treatment timing." Journal of Econometrics 225, no. 2 (2021): 254-277.

Graff Zivin, Joshua S., Yanjun Liao, and Yann Panassié (2020). "How Hurricanes Sweep Up Housing Markets: Evidence from Florida.” NBER Working paper 27542.

Groen, Jeffrey A., Mark J. Kutzbach, and Anne E. Polivka (2020). "Storms and Jobs: The Effect of Hurricanes on Individuals' Employment and Earnings over the Long Term." Journal of Labor Economics 38(3): 653-685.

Hsieh, Chang-Tai, \& Moretti, Enrico (2019). Housing constraints and spatial misallocation. American Economic Journal: Macroeconomics, 11(2), 1-39. 
Hsiang, Solomon M., and Amir S. Jina (2014). "The causal effect of environmental catastrophe on long-run economic growth: Evidence from 6,700 cyclones.” No. w20352. National Bureau of Economic Research.

Hornbeck, Richard (2012). "The enduring impact of the American Dust Bowl: Short-and longrun adjustments to environmental catastrophe." American Economic Review 102, no. 4: 1477-1507.

Hornbeck, Richard, and Keniston, Daniel (2017). "Creative Destruction: Barriers to Urban Growth and the Great Boston Fire of 1872." American Economic Review, 107(6), 13651398.

Jordà, Òscar (2005). "Estimation and inference of impulse responses by local projections.” American economic review 95.1: 161-182.

Kirchberger, Martina (2017). "Natural disasters and labor markets.” Journal of Development Economics 125: 40-58.

Kousky, Carolyn, "Managing shoreline retreat: a US perspective,” Climatic Change, 2014, 124 (1), 9-20.

Kruttli, Mathias S., Roth Tran, Brigitte, and Watugala, Sumudu W. (2023). "Pricing Poseidon: Extreme Weather Uncertainty and Firm Return Dynamics.” Working paper.

Lackner, Stephanie (2019). "Earthquakes and Economic Growth.” FIW Working paper 190.

Lindsay, Bruce R. \& Shawn Reese (2018). "FEMA and SBA Disaster Assistance for Individuals and Households: Application Process, Determinations, and Appeals." Congressional Research Service Report \#45238. June 22, 2018.

Mach, Katharine J, Caroline M Kraan, Miyuki Hino, AR Siders, Erica M Johnston, and Christopher B Field, "Managed retreat through voluntary buyouts of flood-prone properties," Science Advances, 2019, 5 (10), eaax8995.

Martin, Carlos (2018). "The Evidence Base on How CDBG-DR Works for State and Local Stakeholders." Statement before the Subcommittee on Oversight and Investigations Committee on Financial Services, United States House of Representatives.

McCoy, Shawn J., \& Randall P. Walsh (2017). "Wildfire Risk, Salience and Housing Demand," Journal of Environmental Economics and Management 91 (August 2018): 203-228.

Noy, Ilan (2009). "The macroeconomic consequences of disasters." Journal of Development economics 88.2: 221-231. 
Rappaport, J., \& Sachs, J. D. (2003). “The United States as a coastal nation.” Journal of Economic Growth, 8(1), 5-46.

Roback, J. (1982). Wages, rents, and the quality of life. Journal of Political Economy, 90(6), 1257-1278.

Rosen, S. (1979). Wage-based indexes of urban quality of life. Current issues in urban economics, 74-104.

Saiz, Albert. "The geographic determinants of housing supply." The Quarterly Journal of Economics 125, no. 3 (2010): 1253-1296.

Sawada, J., \& Sachs, J. D. (2019). Aggregate Impacts of Natural and Man-Made Disasters: A Quantitative Comparison. International Journal of Development and Conflict, 9, 43-73. Sheldon, Tamara L. and Crystal Zhan (2021, forthcoming). "A Better Place to Call Home: Natural Disasters, Climate Risk, and Regional Migration,” in Robert Sauer, ed., World Scientific Encyclopedia of Global Migration (Singapore: World Scientific Publishing).

Shoag, Daniel (2013). "Using state pension shocks to estimate fiscal multipliers since the great recession." American Economic Review 103, no. 3: 121-24.

Strobl, Eric (2011). "The economic growth impact of hurricanes: evidence from US coastal counties." Review of Economics and Statistics 93.2: 575-589.

Suárez Serrato, Juan Carlos, and Philippe Wingender (2016). "Estimating local fiscal multipliers.” National Bureau of Economic Research Working Paper \#22425.

Vigdor, Jacob (2008). "The economic aftermath of Hurricane Katrina.” Journal of Economic Perspectives 22, no. 4: 135-54.

Von Peter, Goetz, Sebastian Von Dahlen, and Sweta C. Saxena (2012). "Unmitigated disasters? New evidence on the macroeconomic cost of natural catastrophes.” BIS Working Paper No. 394.

USGCRP (2017). “Climate Science Special Report: Fourth National Climate Assessment, Volume I" [Wuebbles, D.J., D.W. Fahey, K.A. Hibbard, D.J. Dokken, B.C. Stewart, and T.K. Maycock (eds.)]. U.S. Global Change Research Program, Washington, DC, USA, 470 pp, doi: 10.7930/J0J964J6.

Wilson, Daniel J. (2012). "Fiscal spending jobs multipliers: Evidence from the 2009 American Recovery and Reinvestment Act." American Economic Journal: Economic Policy 4, no. 3: 251-82. 
Wilson, Daniel J. (2017). "The Impact of Weather on Local Employment: Using Big Data on Small Places.” Federal Reserve Bank of San Francisco Working Paper, 2016-21. 
Figure 1: Natural Disaster Trends and Distribution, 1980 - 2017

(a) Disaster Frequency and Damages

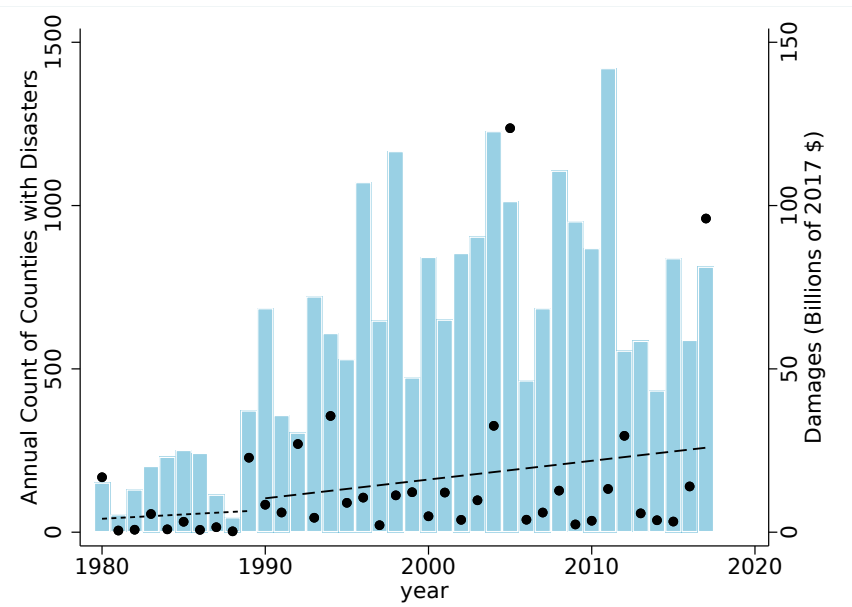

(b) Geographic Distribution of Disasters

Source: Federal Emergency Management Agency (FEMA) and SHELDUS.

Note: The count in panel (a) shows the number of counties each year with at least one disaster declaration. The count in panel (b) shows the number of years with disaster declarations for each county over the period 1980-2017.

Figure 2: Theoretical paths for disaster recovery

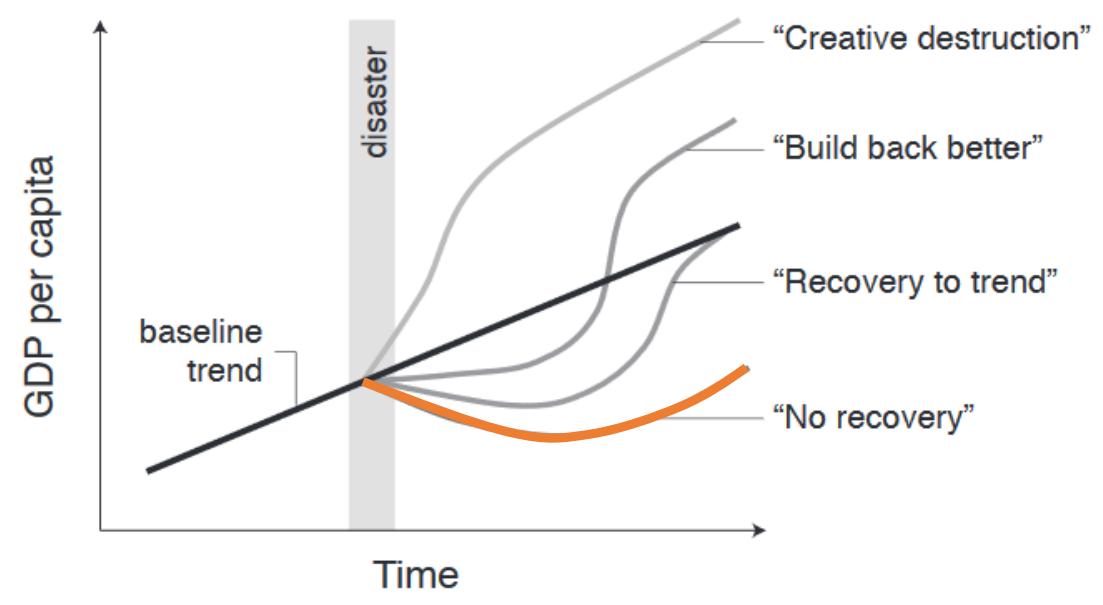

Source: Hsiang and Jina (2014) 
Figure 3: Impulse Responses of Selected Outcomes to Disaster Shocks

(a) Personal Income (per capita)

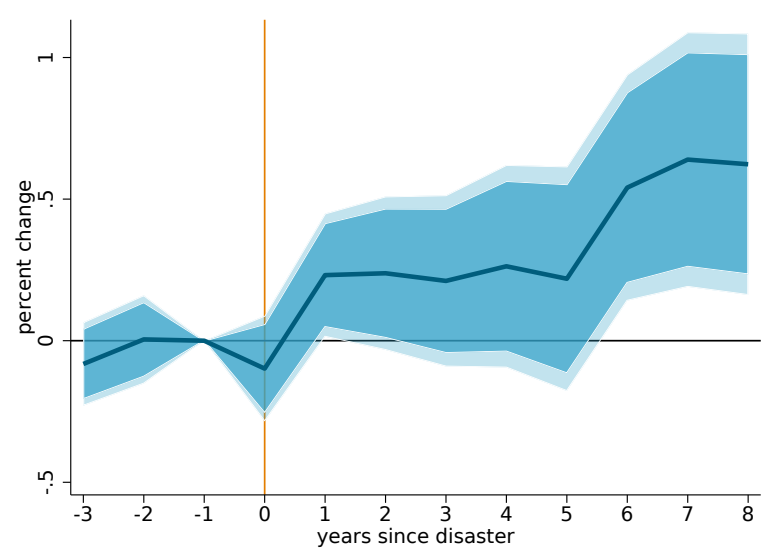

(c) Construction Employment

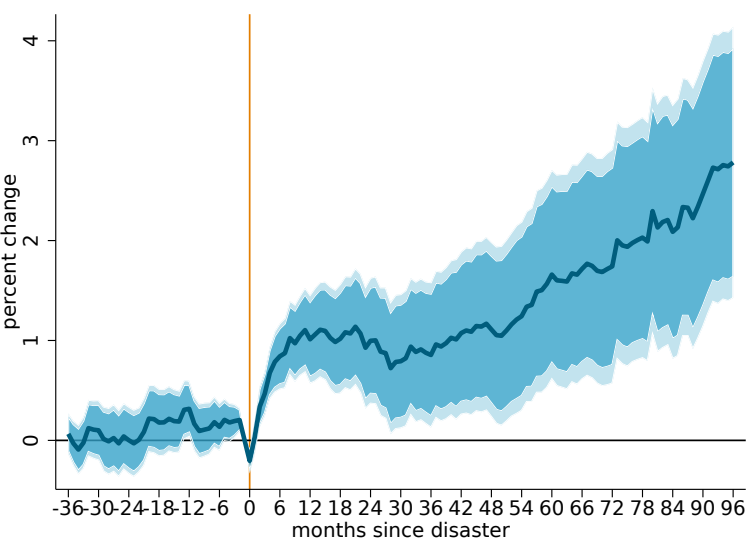

(e) Home Prices

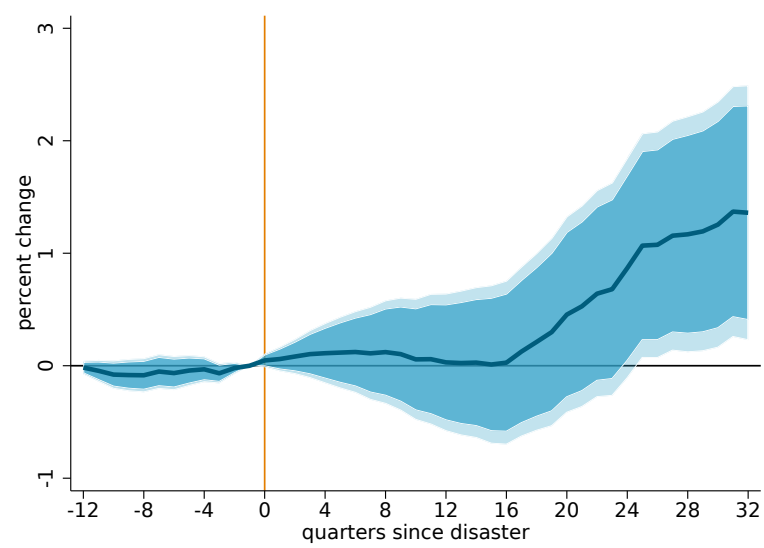

(b) Total Nonfarm Employment

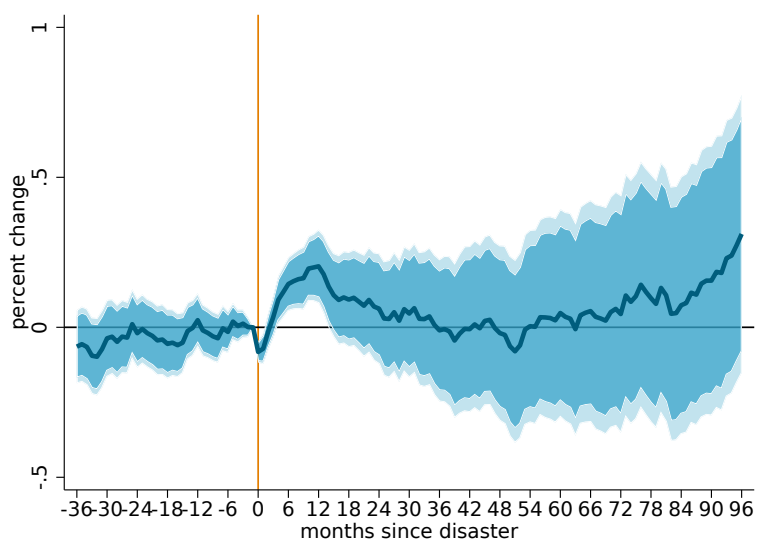

(d) Average Weekly Wages

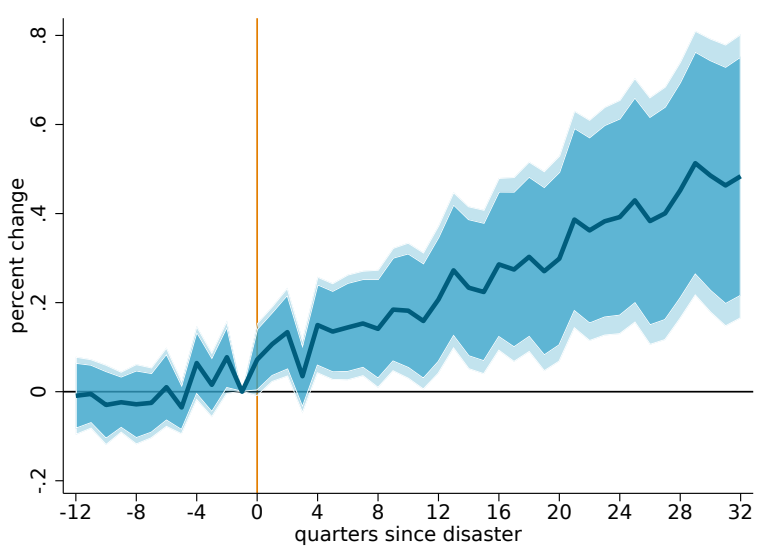

(f) Population

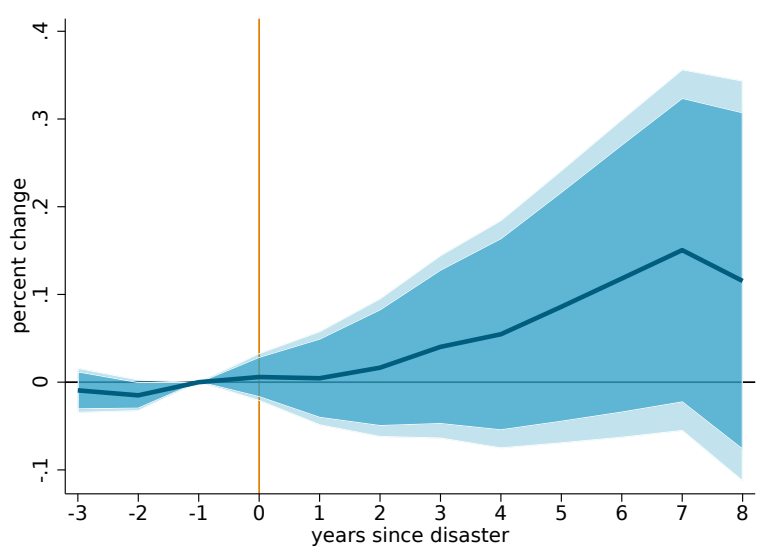

Source: Federal Emergency Management Agency (FEMA), BLS, Census, BEA, and CoreLogic.

Note: These plots show the impulse response functions from estimating equation (1), where the inner shaded regions indicate the 90 percent confidence intervals, and the lighter outer shaded regions indicate the 95 percent confidence intervals. Standard errors are clustered at the county and state-by-time levels. All outcome variables are observed at the county level and modeled as differences in logs between the indicated horizon $(h)$ and the period before the disaster $(-1)$. 
Figure 4: Growing versus declining areas (in terms of house prices)
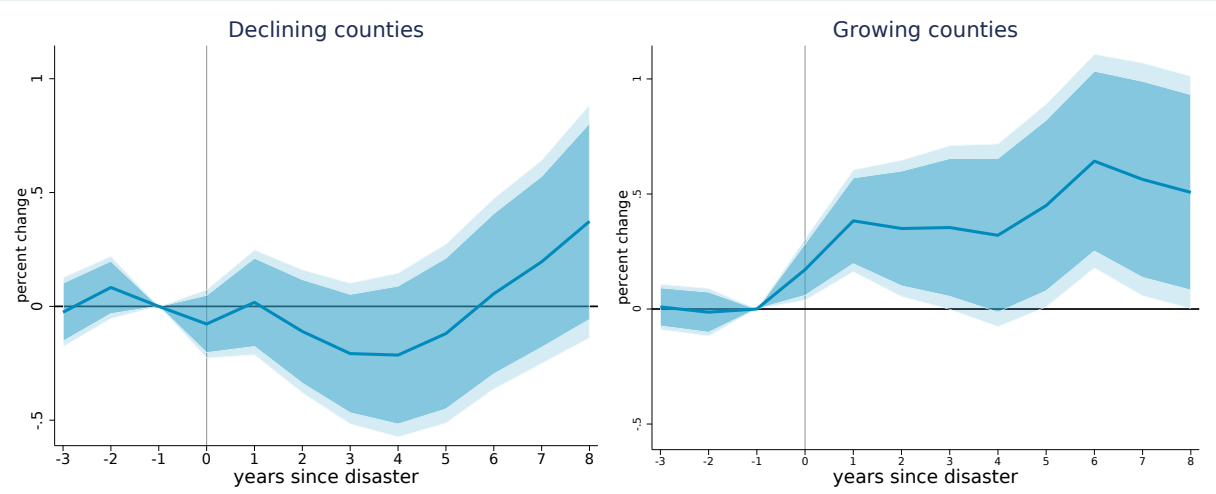

Source: FEMA, BEA, and CoreLogic.

Note: This figure shows the impulse response function from estimating equation (1) interacted with an indicator for whether the house price index was increased (net of inflation) over the three years prior to the disaster. Standard errors are clustered at the county and at the state-by-time levels.

Figure 5: Heterogeneity by housing supply elasticity

(a) House Prices
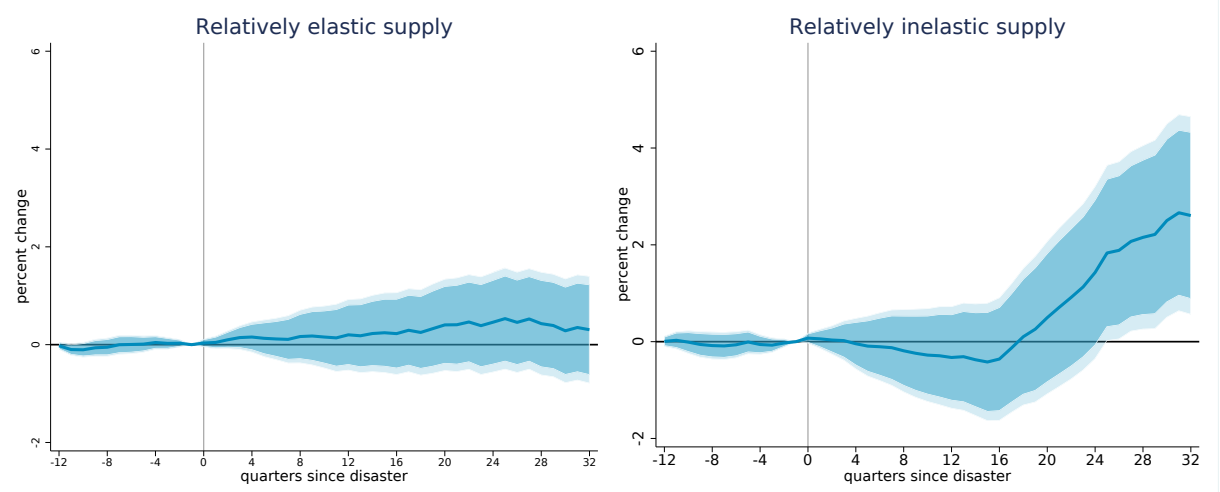

(b) Population
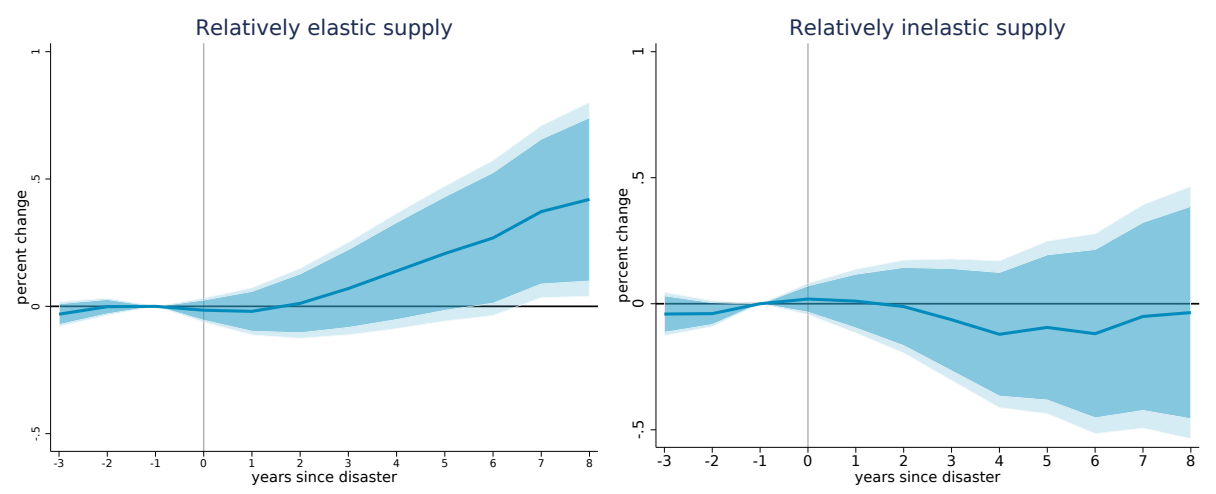

Source: FEMA, BEA, and CoreLogic.

Note: This figure shows the impulse response function from estimating equation (1) interacted with an indicator for whether a county's Saiz elasticity is in the top half of the sample. Standard errors are clustered at the county and at the state-by-time levels. 
Figure 6: Heterogeneous Impulse Responses by Selected Percentiles of Damages Per Capita

(a) Personal Income (per capita)

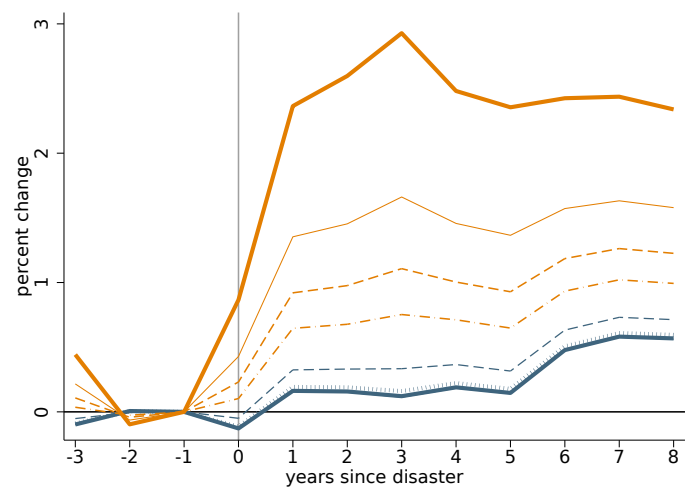

(c) Construction Employment

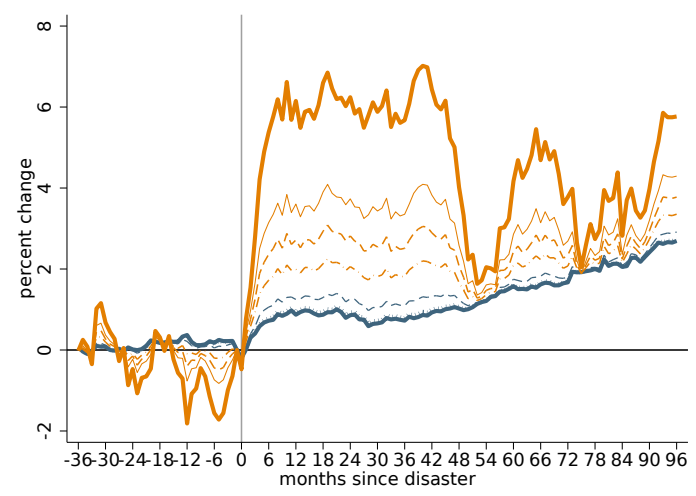

(e) Home Prices

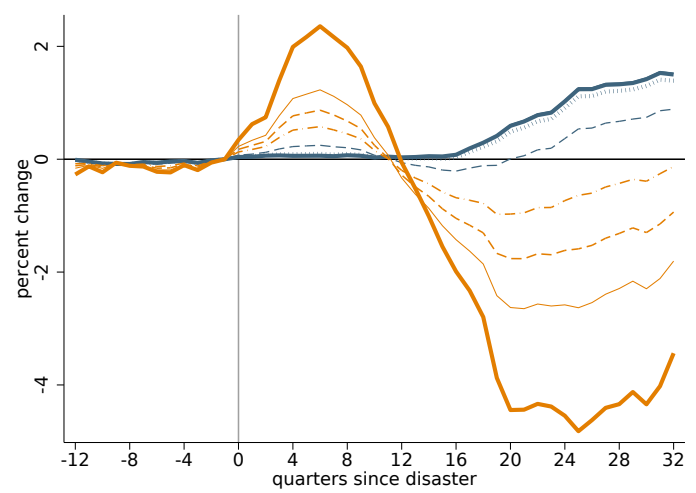

(b) Total Nonfarm Employment

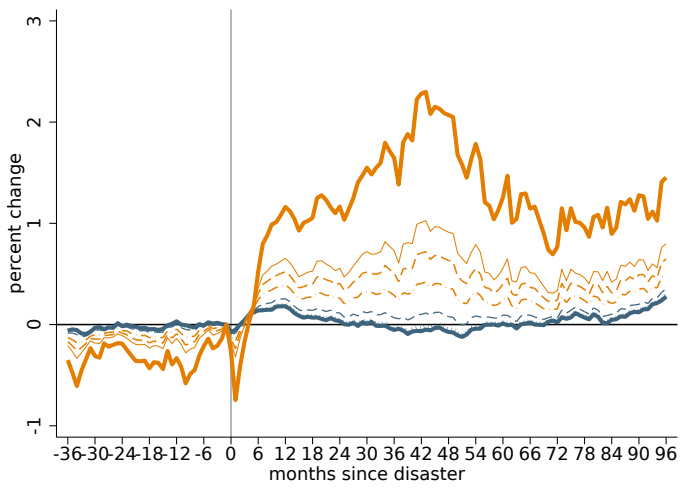

(d) Average Weekly Wages

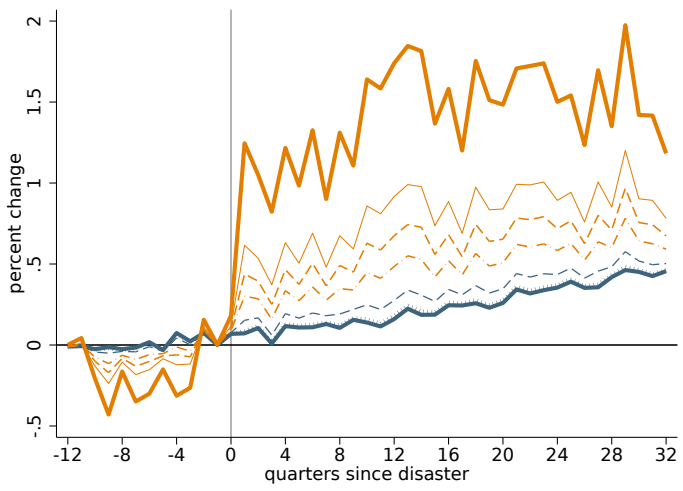

(f) Population

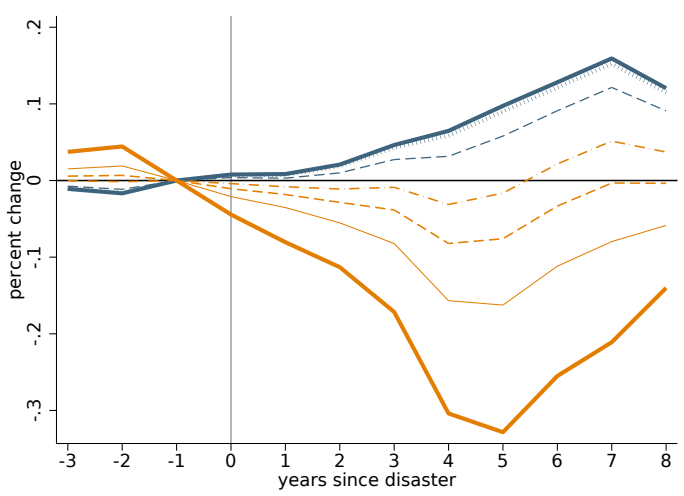

75th ----90 th

Source: FEMA, BLS, Census, BEA, CoreLogic, and SHELDUS.

Note: These plots show the impulse response functions (IRFs) from estimating equation (4), where the percentile lines show the implied IRF for selected percentiles of the damages per capita distribution over all county-month observations with a positive-damages disaster. All variables are observed at the county level and modeled as differences in logs between the indicated horizon $h$ and the period before the disaster $(-1)$. 
Figure 7: Heterogeneous Impulse Responses of Personal Income (p.c.) to Disaster Shocks By Type
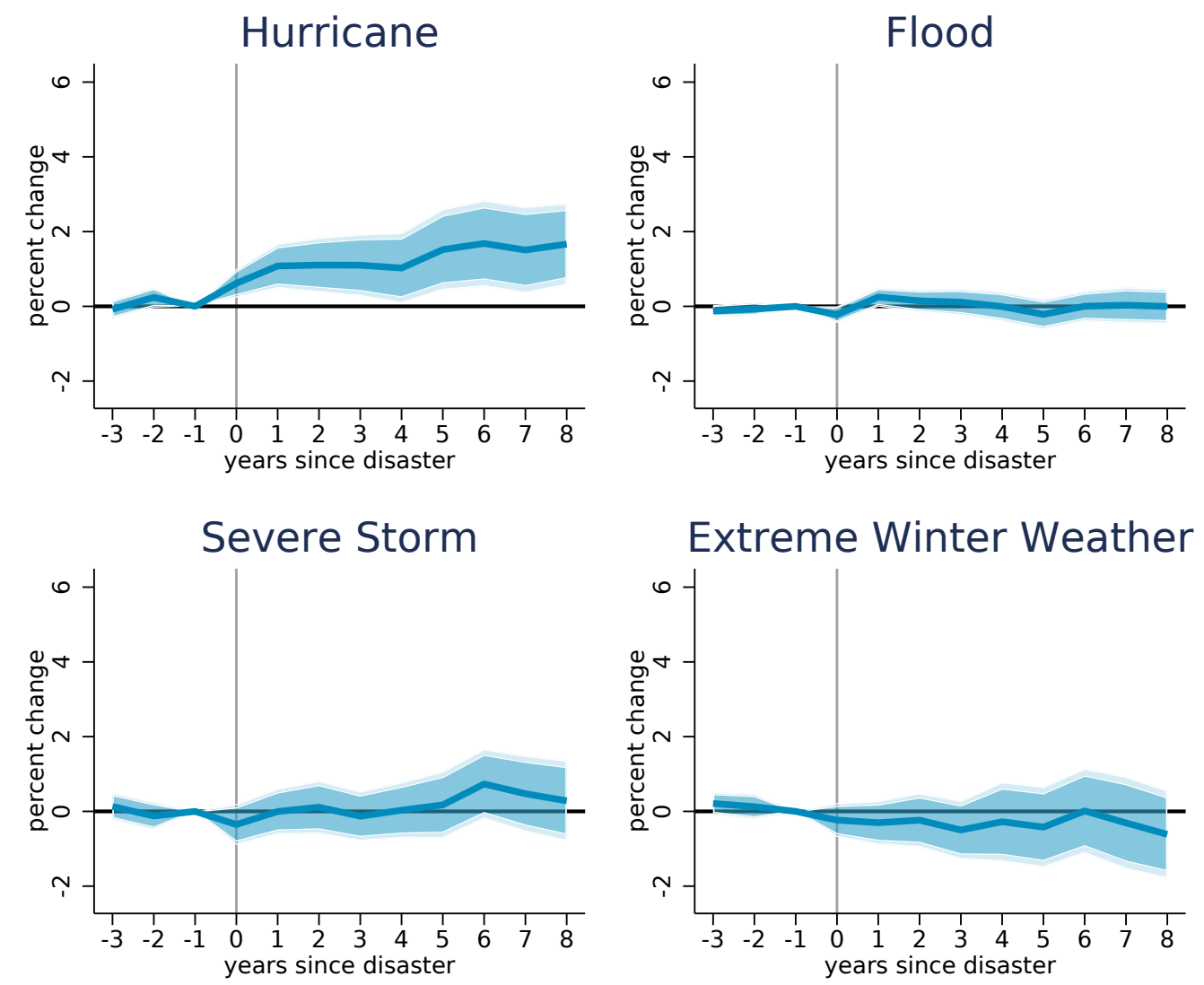

Fire
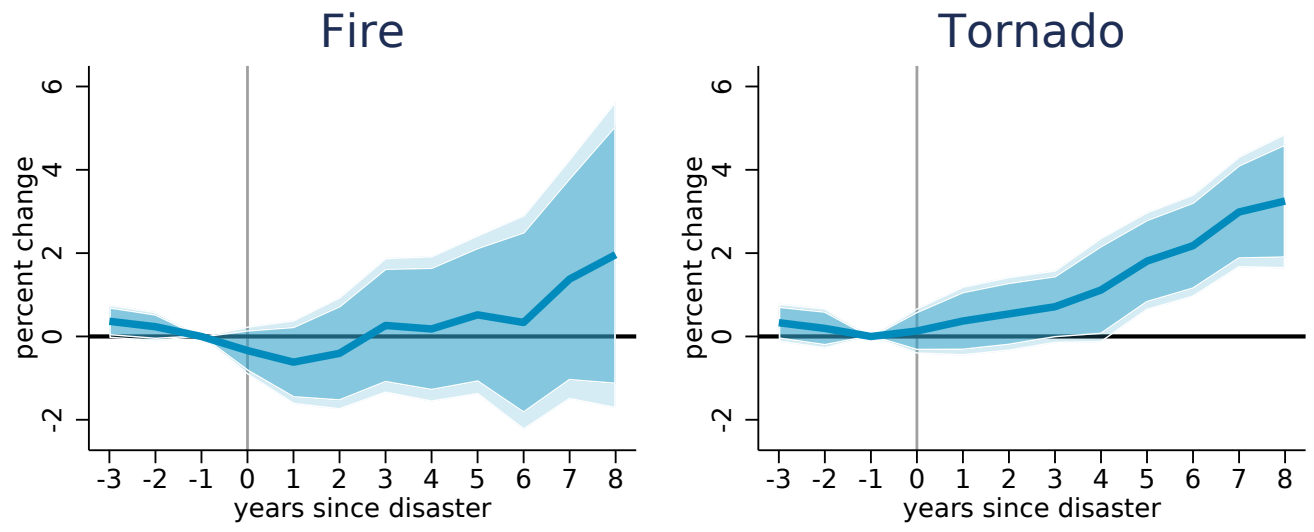

Source: FEMA, SHELDUS, BEA, and Census.

Note: These plots show the impulse response functions from estimating equation (5), where the shaded regions show the 90 and 95 percent confidence intervals. Standard errors are clustered at the county and at the stateby-time levels. The disaster type categories are based on FEMA declaration types and titles, with the flood category excluding floods associated with hurricanes. Each disaster is assigned a single type. However, within a year a county can experience multiple disasters and hence multiple types. Personal income per capita is observed at the county level and modeled as differences in logs between the indicated horizon $(h)$ and the period before the disaster $(-1)$. 
Figure 8: Heterogeneous Impulse Responses of Personal Income (p.c.) to Disaster Shocks

\section{By Initial Personal Income Per Capita}
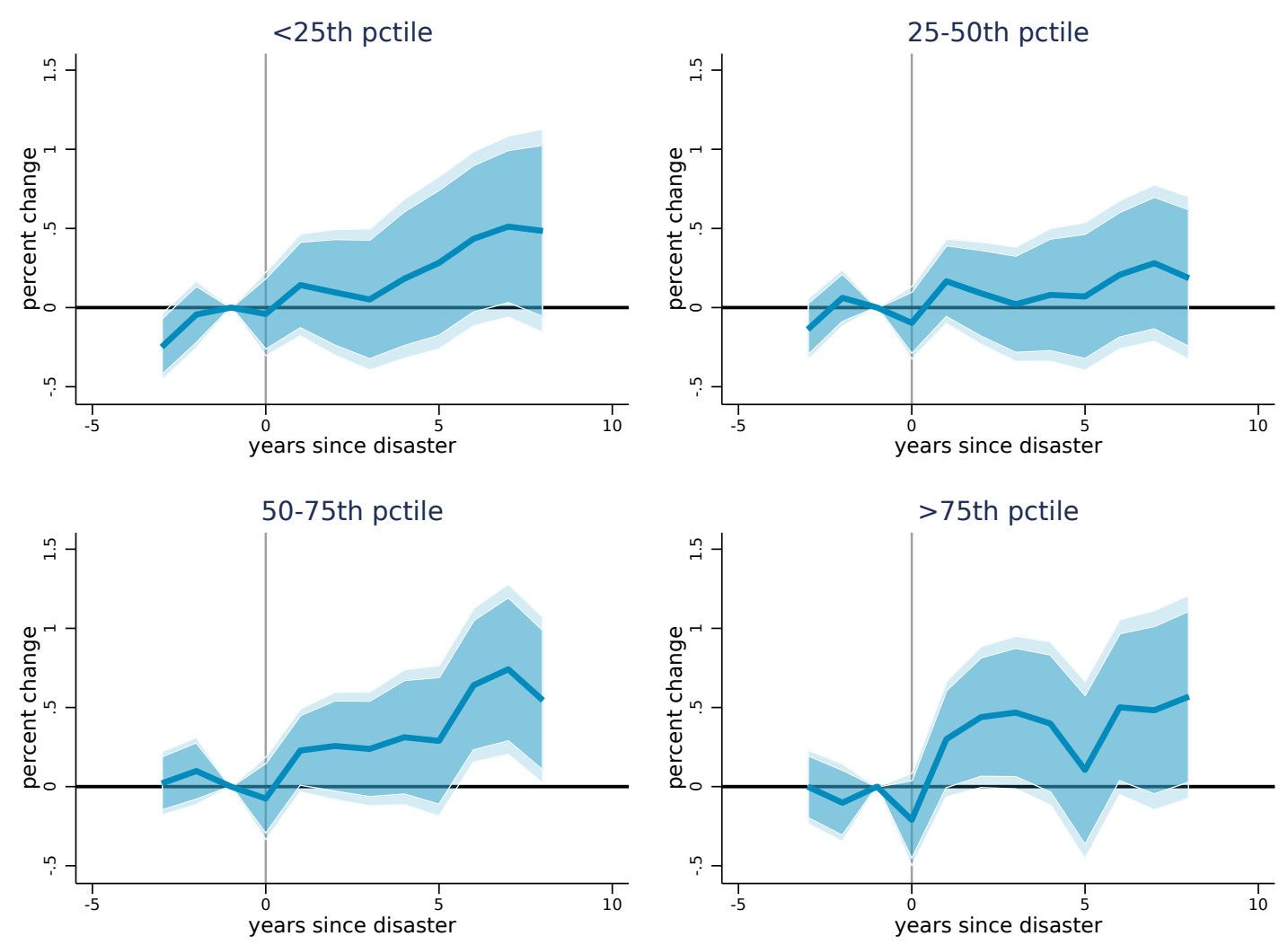

Source: FEMA, SHELDUS, BEA, and Census.

Note: These plots show the impulse response functions from estimating equation (7), where the shaded regions show the 90 and 95 percent confidence intervals. Standard errors are clustered at the county and at the stateby-time levels. The quartiles of initial personal income per capita are based on the personal income in year -1 relative to the national distribution in that year. Personal income per capita is observed at the county level and modeled as differences in logs between the indicated horizon $(h)$ and the period before the disaster $(-1)$. 
Figure 9: Adaptation and Personal Income Per Capita

(a) Heterogeneous Impulse Responses By Local Historical Disaster Exposure
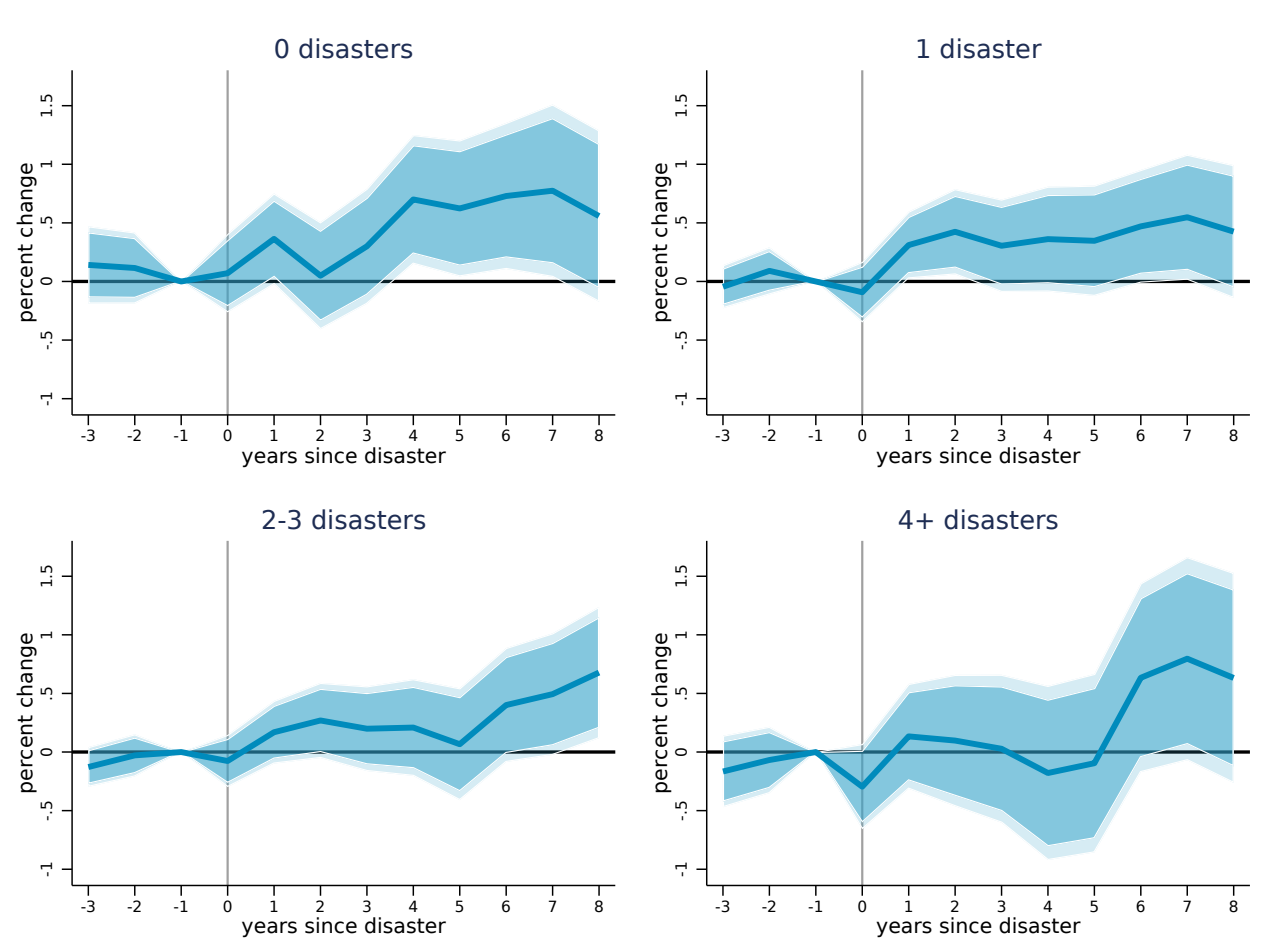

(b) Impulse Responses for Earliest and Latest Years Implied By Time Trend Interaction

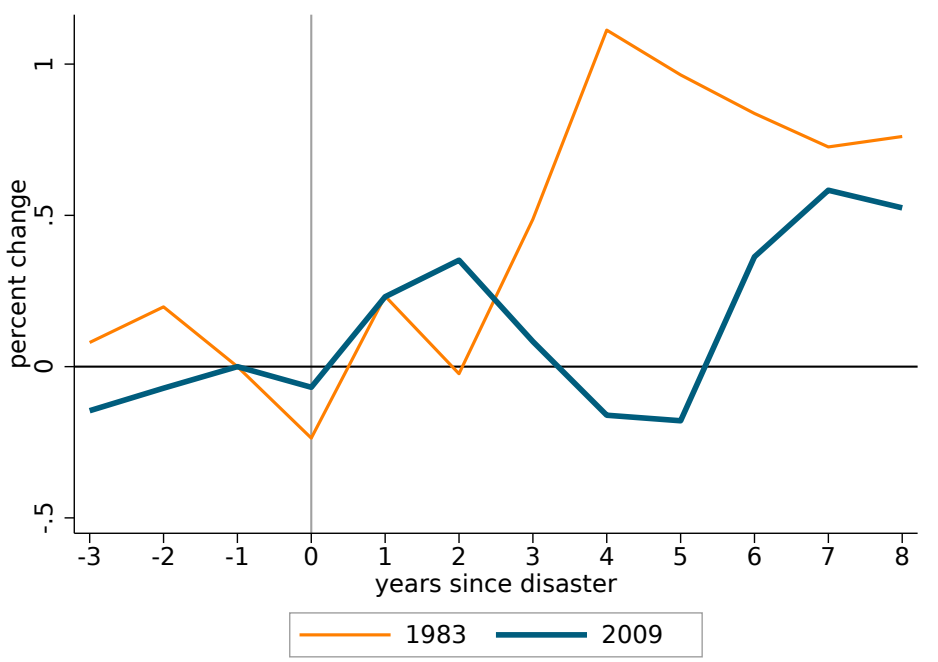

Source: FEMA, SHELDUS, BEA, and Census.

Note: Panel (a) shows the impulse response functions (IRFs) from estimating equation (7), where the shaded regions show the 90 and 95 percent confidence intervals. Standard errors are clustered at the county and at the state-by-time levels. The four categories of historical disaster experience $(0,1,2-3$, and $4+)$ represent the number of years within years -10 to -1 in which a county experienced a disaster. Panel (b) shows the implied IRFs from estimating equation (1) but with an interaction between the disaster dummy and year (i.e., a linear time trend), then calculating the implied IRF for the earliest (1983) and latest year (2009) possible given our sample period and leads and lags. Personal income per capita is observed at the county level and modeled as differences in logs between the indicated horizon $(h)$ and the period before the disaster $(-1)$. 
Figure 10: Impacts of Own-county Disasters vs. Spatially-Lagged Disasters on Personal Income (p.c.)

Results for spatial lags of varying distance bands

(a) Disasters up to 199 Miles Away

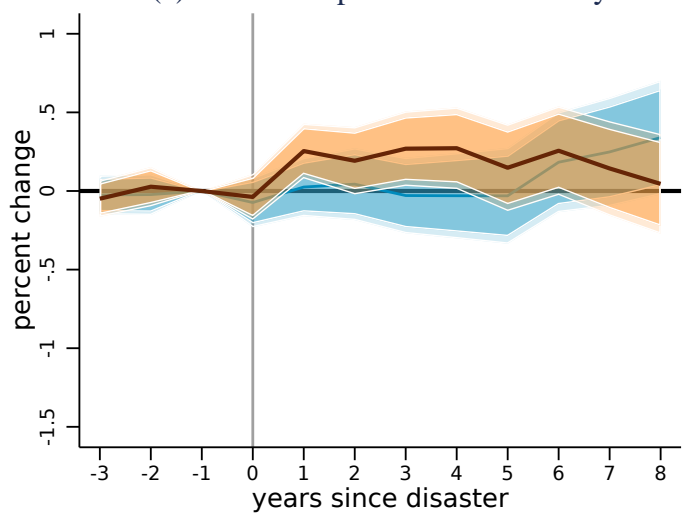

(c) Disasters 400-599 Miles Away

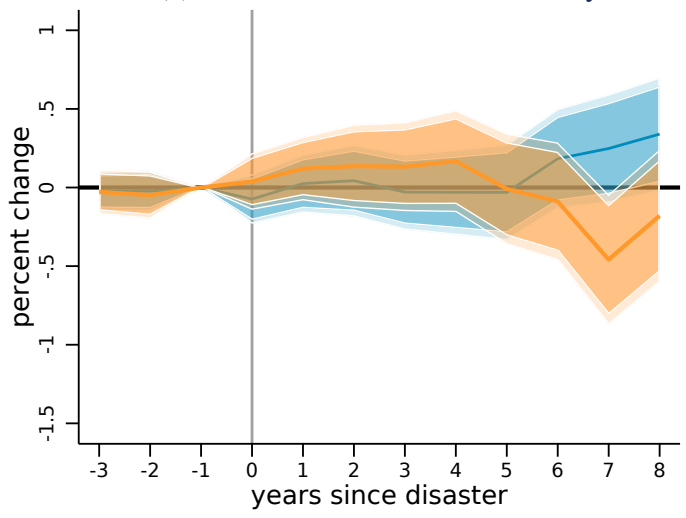

(b) Disasters 200-399 Miles Away

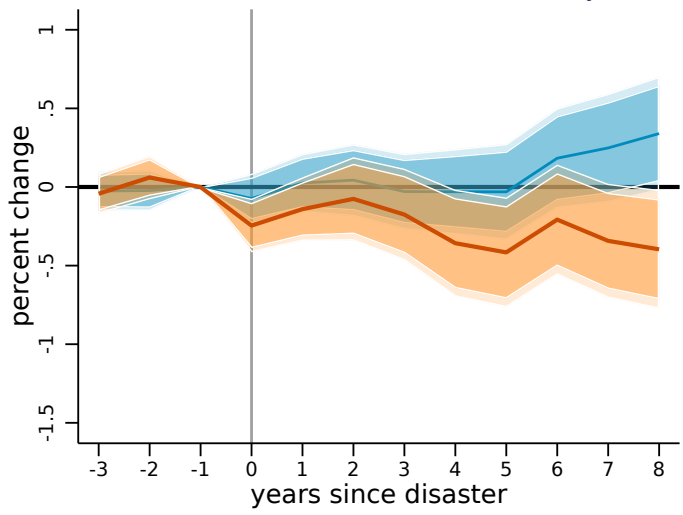

(d) Net Effect Within 600 mile range

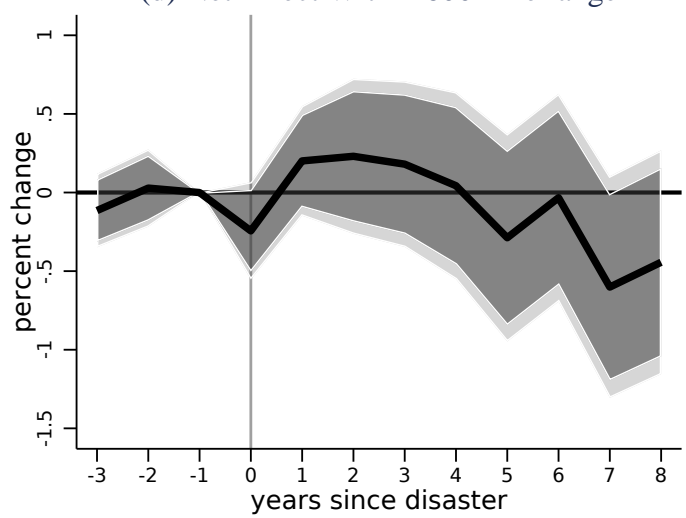

\begin{tabular}{|llll|}
- & Other Counties up to 199 miles away & - & Own-County \\
- & Other Counties 200-399 miles away & Net Effect \\
- & Other Counties 400-599 miles away & & \\
\hline
\end{tabular}

Source: FEMA, SHELDUS, Census, and BEA.

Note: Panels (a)-(c) show the impulse response functions (IRFs) from estimating equation (8), where the shaded regions show the 90 and 95 percent confidence intervals. Standard errors are clustered at the county and at the state-by-time levels. The thin blue curve (repeated in each panel) reflects the IRF estimated for counties directly experiencing a disaster. The orange curves depict the IRFs for the counties within the indicated mile ranges of counties experiencing disasters. The intensity of treatment for the orange curves is the share of population within each band that has experienced a disaster in period 0. Each of the orange curves has been rescaled by the mean population share for positive observations within the band. Thus the curves represent the average effect on counties having at least one county within the given range experience a disaster in period 0 . Panel (d) shows the net effect on personal income within these bands, where each coefficient has been rescaled by the variable's unconditional mean. Personal income per capita is observed at the county level and modeled as differences in logs between the indicated horizon $(h)$ and the period before the disaster $(-1)$. 
Figure 11: State-Level Impulse Response of Personal Income (p.c.) to Disaster Shocks

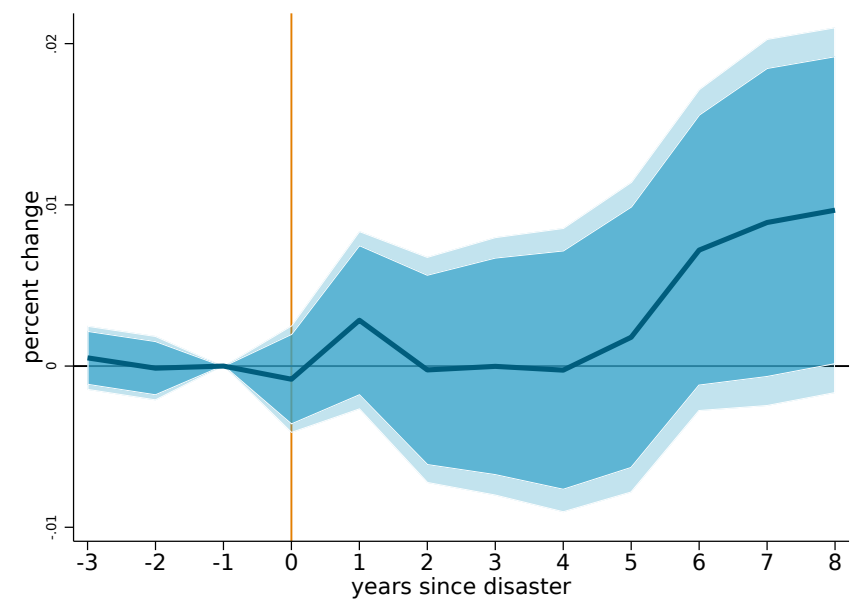

Source: FEMA, SHELDUS, BLS, Census, BEA, and CoreLogic.

Note: This figure shows the impulse response function from estimating equation (1) on state-aggregated data, where the treatment is the share of the state's population living in counties hit by disasters in a given year. The inner shaded region indicates the 90 percent confidence intervals, and the lighter outer shaded region indicates the 95 percent confidence intervals. Personal income is aggregated at the state level and modeled as differences in logs between the indicated horizon $(h)$ and the period before the disaster $(-1)$. Standard errors allow for clustering by state. The regressions include Census division-by-time fixed effects and state fixed effects. 


\section{Online Appendix A - Not For Publication}

Table A1: Variable Descriptions

\begin{tabular}{lccccc}
\hline \multicolumn{1}{c}{ Variable } & Frequency & Form & Winsorized & Per capita & Source \\
\hline Personal income & annual & $\log$ & no & yes & BEA \\
Total nonfarm employment & monthly & $\log$ & $0.5,99.5$ & no & QCEW \\
Construction employment & monthly & $\log$ & $0.5,99.5$ & no & QCEW \\
Average weekly wages & quarterly & $\log$ & $0.5,99.5$ & no & QCEW \\
House price index & quarterly & $\log$ & $0.5,99.5$ & no & CoreLogic \\
Population & annual & $\log$ & $0.5,99.5$ & no & Census \\
Government transfers & annual & $\log$ & no & yes & BEA \\
Income maintenance transfers & annual & $\log$ & no & yes & BEA \\
UI transfers & annual & $\log$ & no & yes & BEA \\
FEMA IHP aid & annual & $\log (1+\cdot)$ & $0.5,99.5$ & yes & FEMA \\
SBA disaster loans & annual & $\log (1+\cdot)$ & $0.5,99.5$ & yes & SBA \\
NFIP payouts & annual & $\log (1+\cdot)$ & $0.5,99.5$ & yes & FEMA \\
Wage \& salary income & annual & $\log$ & no & yes & BEA \\
Poverty rate & annual & $\log$ & no & yes & SAIPE \\
Migration & annual & percent & no & yes & IRS SOI \\
Industry-based hourly wages & annual & $\log$ & yes & no & CBP, CEPR \\
Damages & monthly & $\operatorname{linear}$ & no & yes & SHELDUS \\
FEMA disasters & monthly & indicator & N $/ A$ & N/A & FEMA \\
\hline
\end{tabular}

Note: UI stands for unemployment insurance, FEMA for Federal Emergency Management Agency, IHP for Individual and Household Program (aid), SBA for the Small Business Administration, NFIP for the National Flood Insurance Program, BEA for the Bureau of Economic Analysis, QCEW for the Quarterly Census of Employment and Wages, SAIPE for the Small Area Income and Poverty Estimates (at the Census Bureau), IRS SOI for the Internal Revenue Service Statistics on Income, CBP for the Census Bureaus's County Business Pattern data, CEPR for the Center for Economic Policy Research, and SHELDUS for the Spatial Hazard Events and Losses Database for the United States.

Data on IHP aid and NFIP payments are aggregated to annual frequency to be comparable to data on other government transfer income. Also, IHP aid and NFIP payments data reflect the time period of the disaster with which they are associated; data on when they are paid out is not available. The annual SBA Loan data are based on the fiscal year ending September 30 of the indicated calendar year. We examine $\log (1+\cdot)$ form for the aid variables to address the very high share of observations with zero aid. 
Table A2: Summary Statistics

\begin{tabular}{lcccccc}
\hline \hline & Mean & SD & Min & Median & Max & N \\
\hline Levels & & & & & & \\
Personal income (per capita) & 28,702 & 8,631 & 6,494 & 27,328 & 183,918 & 109,486 \\
Total nonfarm employment & 30,830 & 117,776 & 0 & 5,612 & $3,875,009$ & $1,289,249$ \\
Construction employment & 1,878 & 6,383 & 0 & 317 & 181,710 & 932,721 \\
Average weekly wages & 602 & 140 & 0 & 581 & 11,996 & 430,974 \\
Home price index & 101 & 43.1 & 19.6 & 95.2 & 369 & 183,142 \\
Population & 90 & 291 & .055 & 24.7 & 10,164 & $1,364,091$ \\
Disaster indicator (monthly) & .0199 & .14 & 0 & 0 & 1 & $1,364,100$ \\
Per capita damages & 228 & 5,522 & 0 & 1.02 & 848,616 & 27,172 \\
& & & & & & \\
First differences of logs & & & & & & \\
Personal income per capita & 1.57 & 5.81 & -103 & 1.49 & 146 & 106,296 \\
Total nonfarm employment & .0868 & 3.36 & -16.9 & .207 & 16.1 & $1,285,096$ \\
Construction employment & .182 & 9.37 & -40.5 & .157 & 38.8 & 796,369 \\
Average weekly wages & .154 & 7.18 & -22.8 & .235 & 22 & 426,808 \\
Home price index & .878 & 1.99 & -23.8 & .882 & 20.3 & 181,749 \\
Population & .467 & 1.62 & -4.56 & .341 & 6.88 & 110,409 \\
\hline \hline
\end{tabular}

Source: QCEW, Census, CoreLogic, BEA, Federal Emergency Management Agency (FEMA), and the Spatial Hazard Events and Losses Database for the United States (SHELDUS).

Note: See Table 1 and its notes for description of variable names and further details. The first differences of log variables have been rescaled by 100 so that they show percentage point values. The contemporaneous per capita damages may equal zero if there are positive damages observed in the following month. Income and wages are reported in 2010 US dollars. Damages are reported in 2017 US dollars. 
Figure A1: Distribution of Disaster Declarations

Per Capita Damages at or Above 99th Percentile

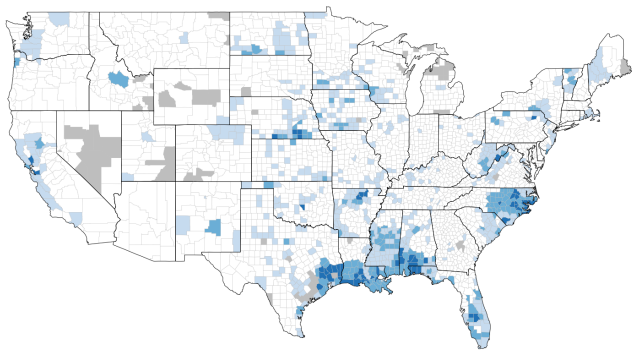

Hurricanes (13\%)

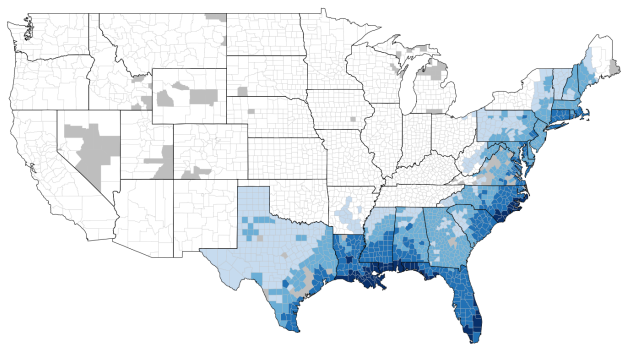

Severe Storms (13\%)

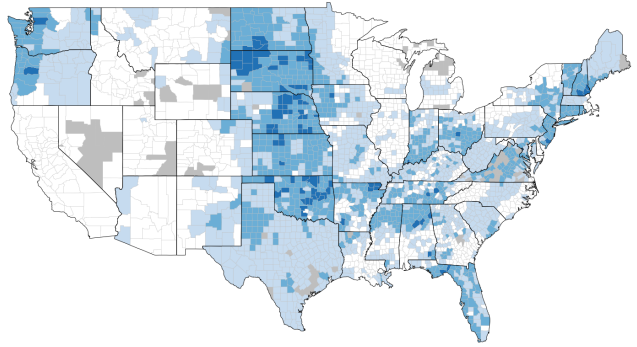

Tornadoes (3\%)

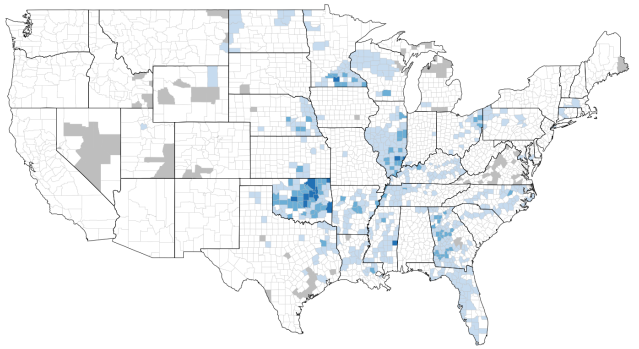

Floods (58\%)
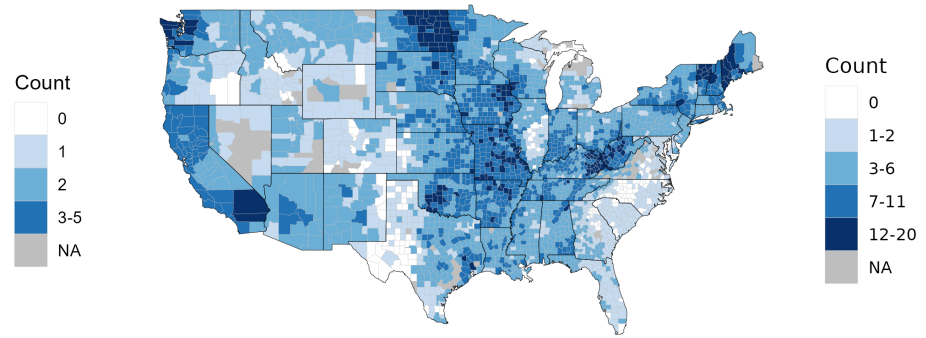

Hurricanes with Associated SHELDUS Damages (12\%)

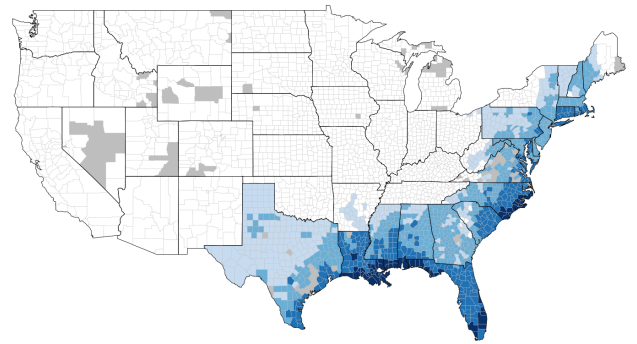

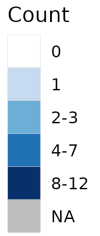

Severe Winter Weather (10\%)

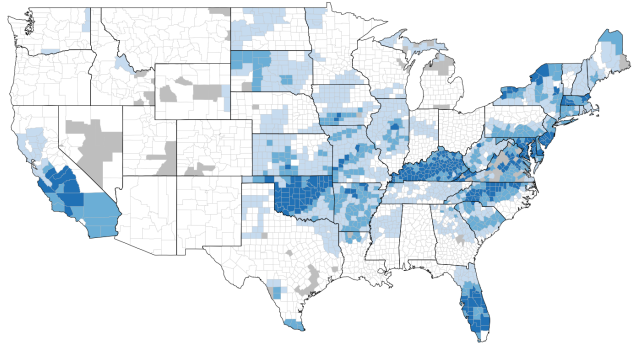

\begin{tabular}{c} 
Count \\
0 \\
0 \\
1 \\
2 \\
\hline $3-6$ \\
\hline NA \\
\hline
\end{tabular}

Fires (3\%)

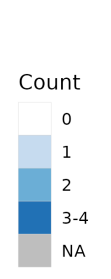

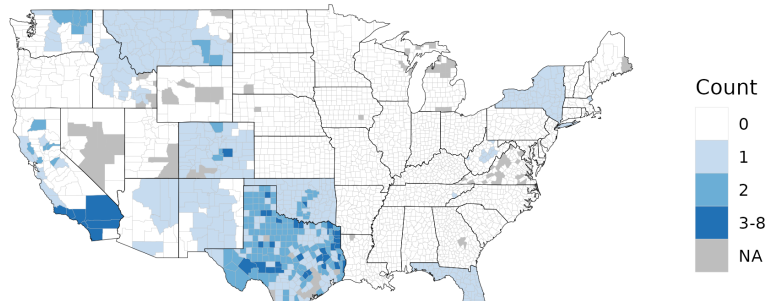

Source: FEMA, SHELDUS.

Note: The All Disaster Types map shows counts of months with at least one disaster with damages reported in SHELDUS. The Per Capita Damages at or Above 99th Percentile map shows the number of months a county's disasters had per capita damages in the 99th percentile of those with FEMA disaster declarations from 1980 to 2017. The remaining maps show the counts of months in which the disaster type was declared in a given county with some hierarchical ordering. If a county experienced flooding due to a hurricane, that will show up only on the hurricane map. If a county receives two separate disaster declarations in a month, one for a hurricane and one for a flood not caused by the hurricane, this will also only show up on the hurricane map. 
Figure A2: Alternative Specification - County-Specific Linear Time Trend

$$
\text { Impacts - All Disasters }
$$

(a) Personal Income (per capita)

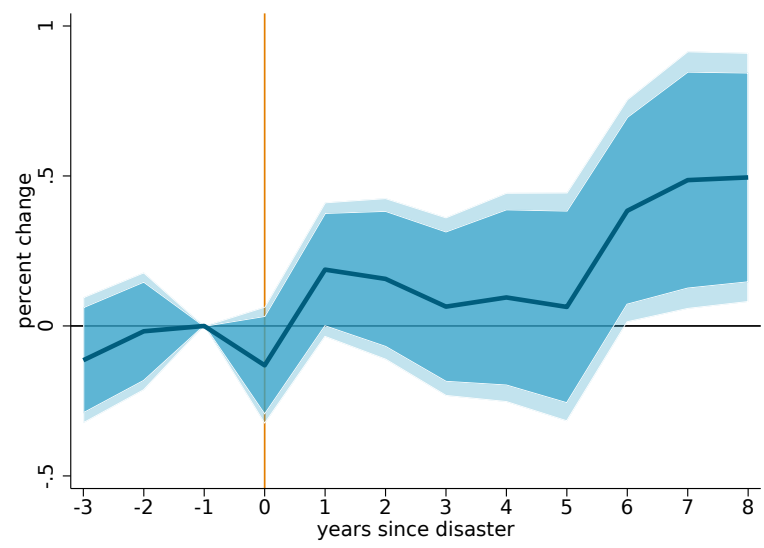

(c) Construction Employment

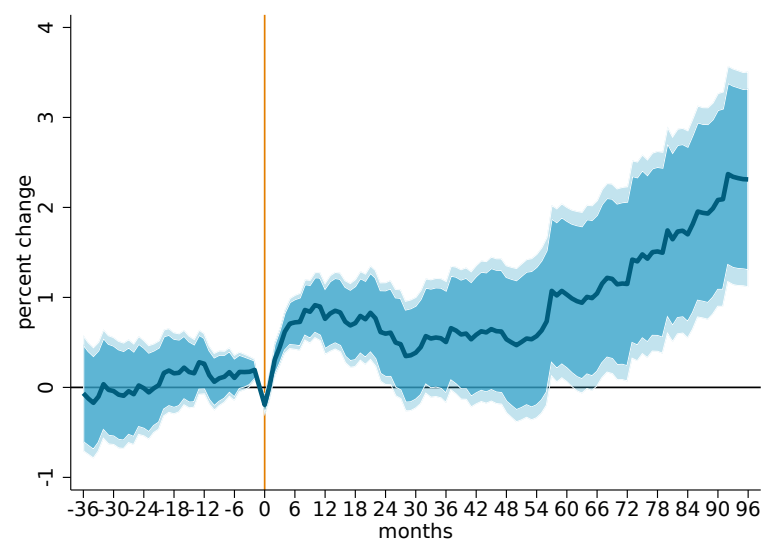

(e) Home Prices

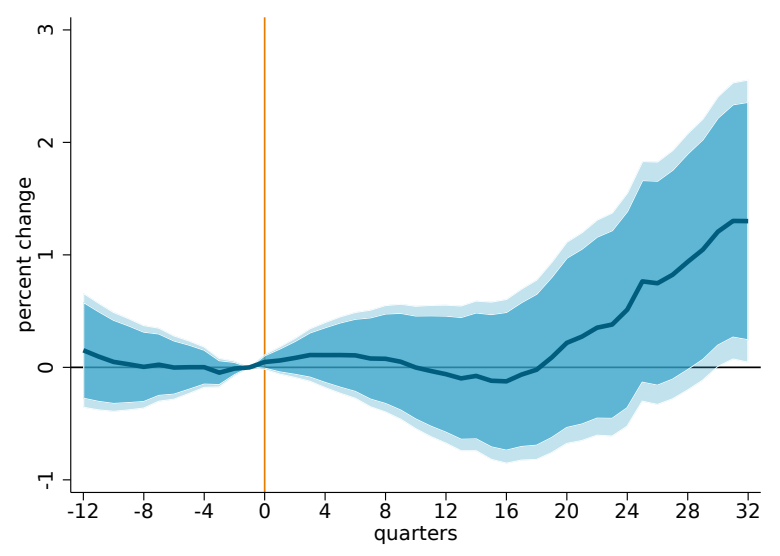

(b) Total Nonfarm Employment

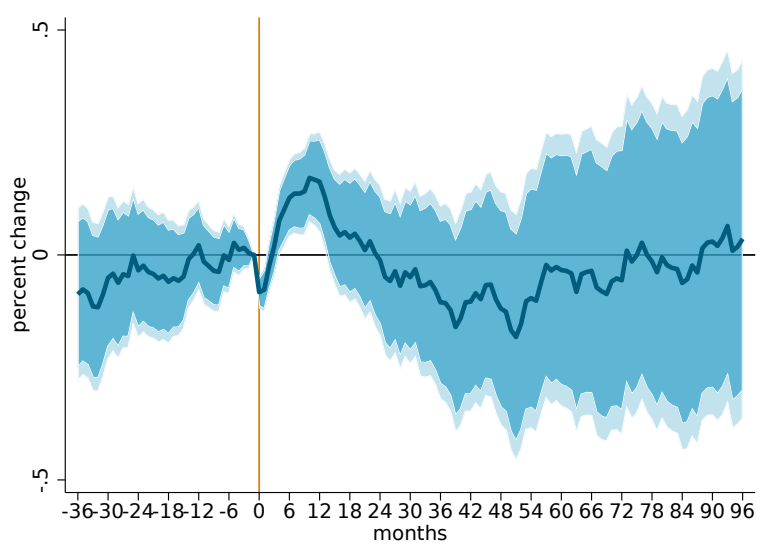

(d) Average Weekly Wages

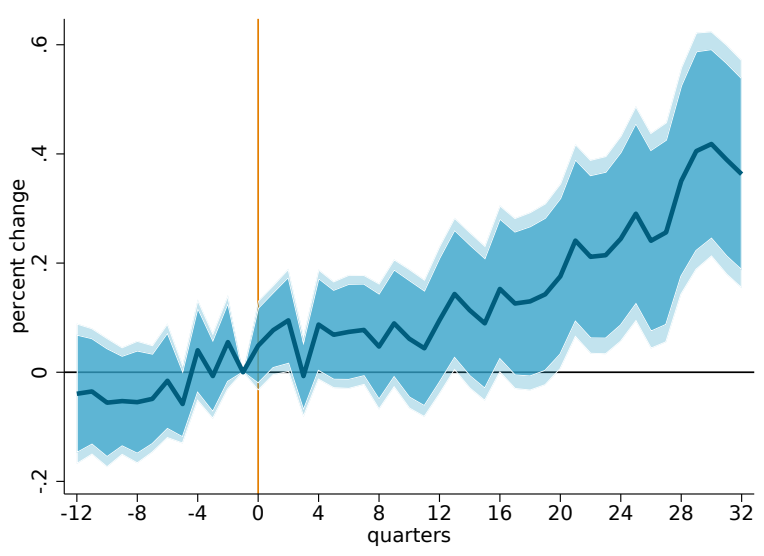

(f) Population

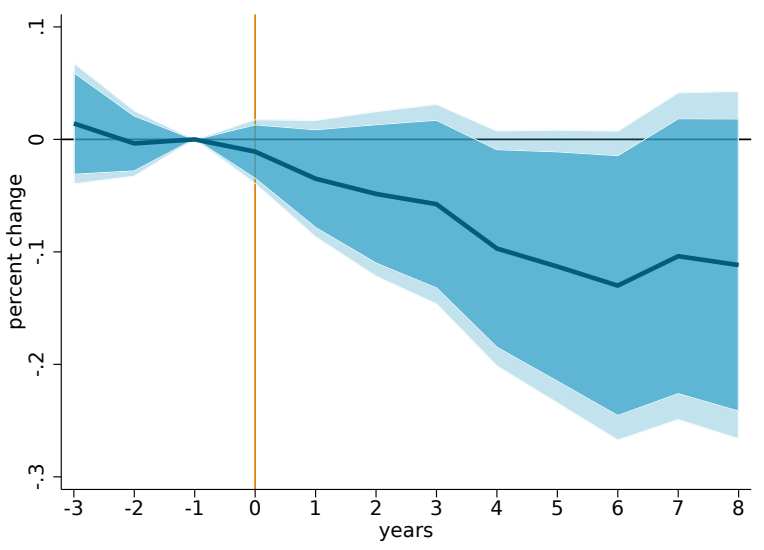

Source: FEMA, SHELDUS, BLS, Census, BEA, and CoreLogic.

Note: These plots show the impulse response functions from estimating an alternative to equation (1), the equation (2) pre-trend term has been replaced with a county-specific time trend. The inner shaded regions indicate the 90 percent confidence intervals, and the lighter outer shaded regions indicate the 95 percent confidence intervals. Standard errors are clustered at the county and at the state-by-time levels. All variables are observed at the county level and modeled as differences in logs begtween the indicated horizon $(h)$ and the period before the disaster $(-1)$. 
Figure A3: Impulse Response of Poverty Rate to Disaster Shocks

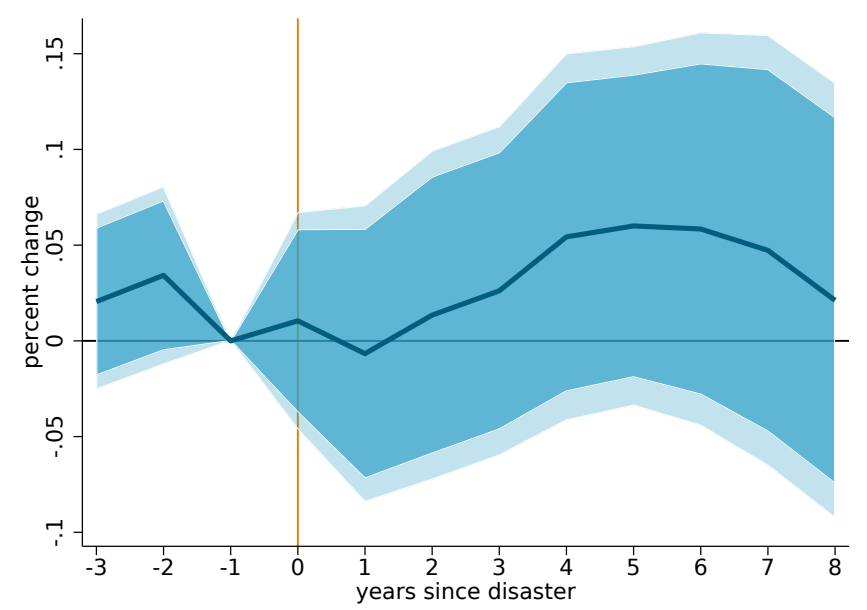

Source: FEMA, SHELDUS, and the Census Bureau's Small Area Income and Poverty Estimates (SAIPE) program.

Note: Figure shows the impulse response function from estimating equation (1) where the dependent variable is the poverty rate. The inner shaded regions indicate the 90 percent confidence intervals, and the lighter outer shaded regions indicate the 95 percent confidence intervals. Standard errors are clustered at the county and at the state-by-time levels. 
Figure A4: Alternative Specifications for Trends

\section{Impulse Response of Personal Income (p.c.) to Disaster Shocks}

(a) Baseline - Control for Pre-trend

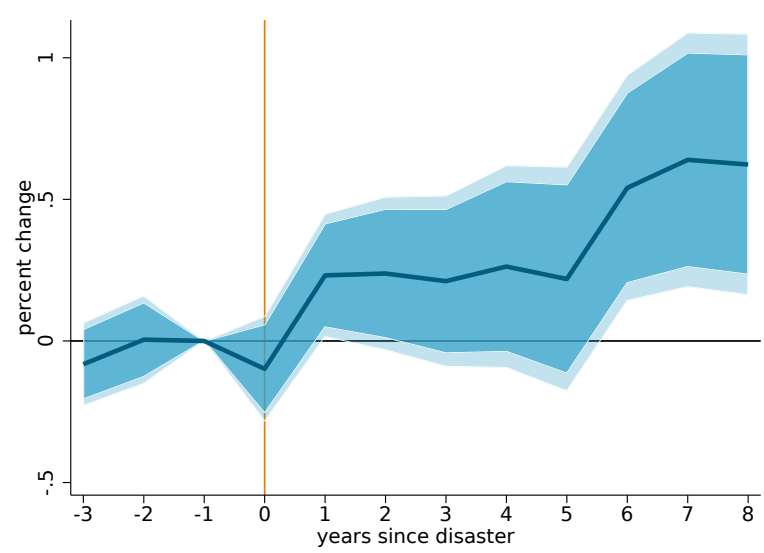

(c) County-Specific Linear Time Trend

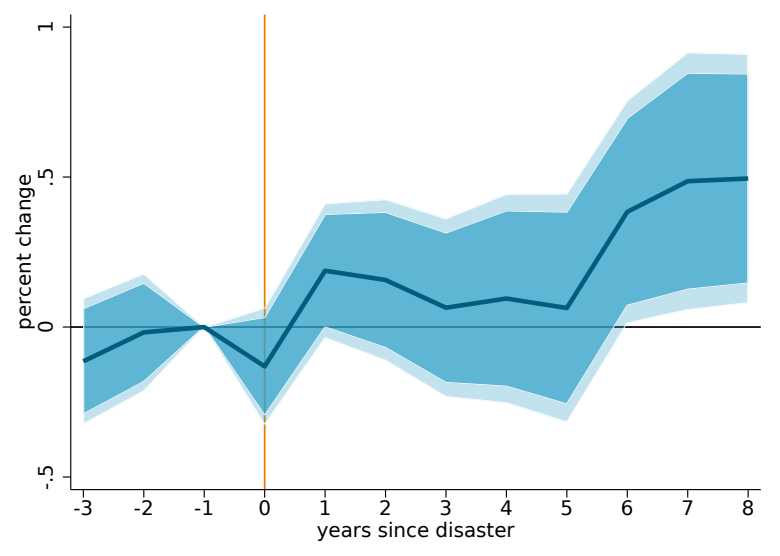

(b) No Pre-trend

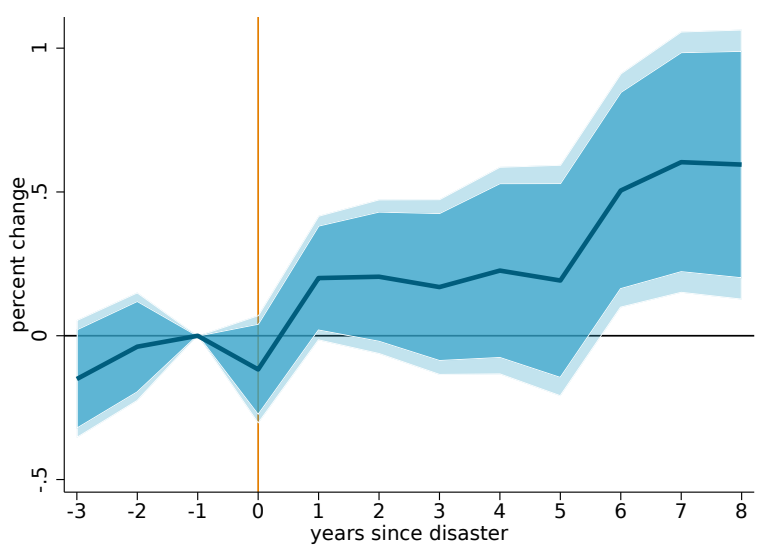

(d) Control for 3 lags of $\mathrm{Y} / \mathrm{Y}$ Growth

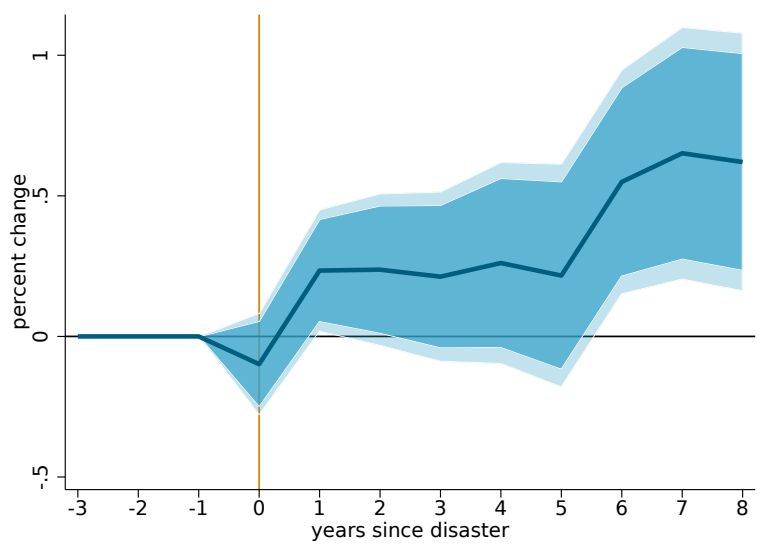

Source: FEMA, SHELDUS, BLS, Census, BEA, and CoreLogic.

Note: These plots show the impulse response functions from estimating equation (1), where the equation (2) pre-trend term has been replaced with either no trend, a county-specific time trend, or three lags of annual income p.c. growth. The inner shaded regions indicate the 90 percent confidence intervals, and the lighter outer shaded regions indicate the 95 percent confidence intervals. Standard errors are clustered at the county and at the state-by-time levels. All variables are observed at the county level and modeled as differences in logs between the indicated horizon $(h)$ and the period before the disaster $(-1)$. 
Figure A5: Coefficient Plots for Baseline Regressions with Sample Restrictions According to Disaster Frequency

(a)

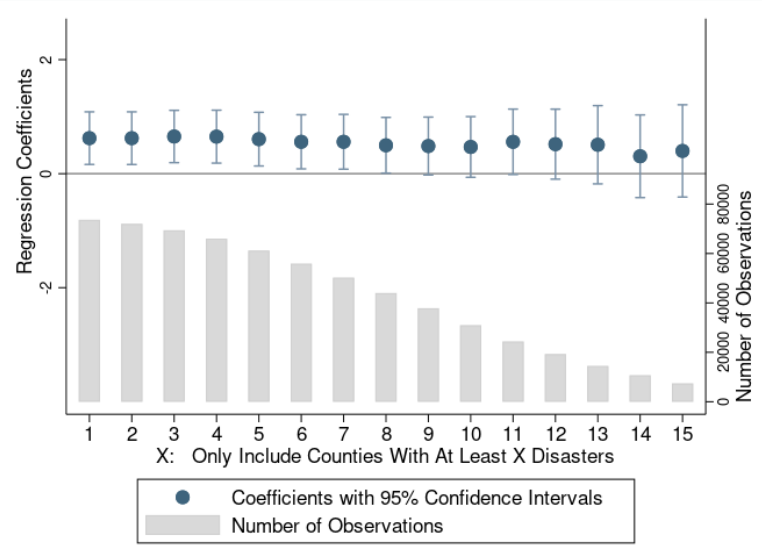

(b)

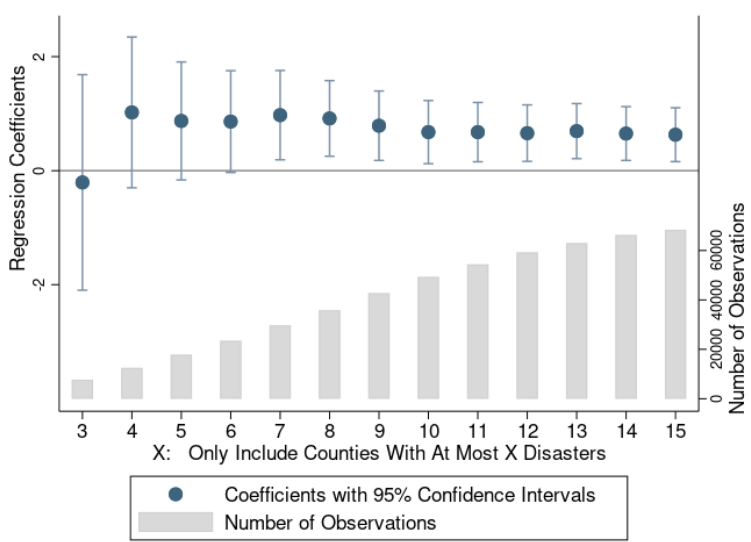

Source: FEMA, BEA.

Note: These plots show estimates of impacts of natural disasters on personal income eight years post-disaster when imposing restrictions on the sample. The dots mark the point estimates, and the lines indicate the 95 percent confidence intervals. The bars below each point estimate indicate the sample size. The results are based on estimating equation (1) for a horizon of eight years after a disaster. Standard errors are clustered at the county and state-by-time levels. Personal income per capita is observed at the county level and modeled as difference in logs between eight years after the disaster and the year before the disaster $(-1)$.

Figure A6: Conditioning on Positive Damages

Impulse Response of Personal Income (p.c.) to Disaster Shocks

(a) Baseline - All FEMA Disasters

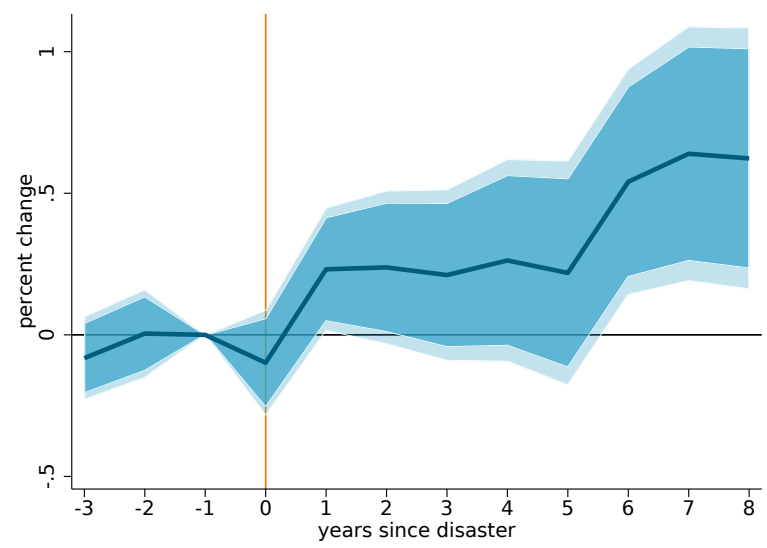

(b) Disasters with Positive Damages

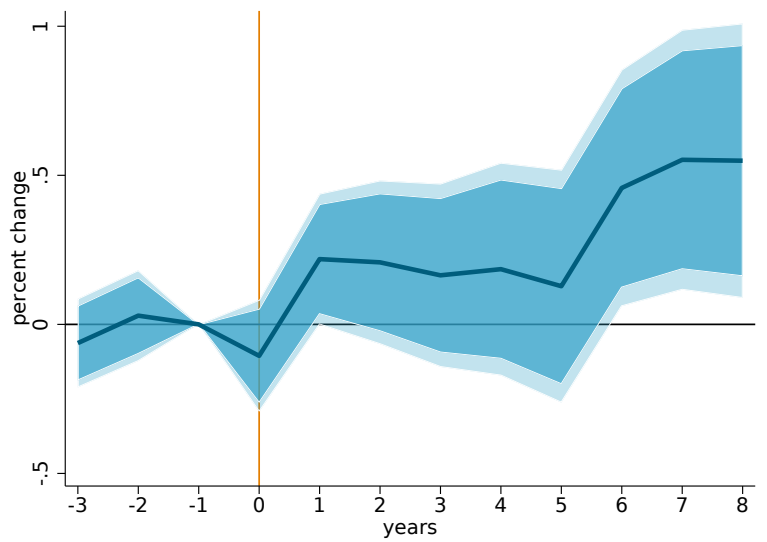

Source: Federal Emergency Management Agency (FEMA), Census, and BEA.

Note: These plots show the impulse response functions from estimating equation (1), where the inner shaded regions indicate the 90 percent confidence intervals, and the lighter outer shaded regions indicate the 95 percent confidence intervals. Standard errors are clustered at the county and state-by-time levels. All outcome variables are observed at the county level and modeled as differences in logs between the indicated horizon $(h)$ and the period before the disaster $(-1)$. 
Figure A7: Impulse Response of Industry-Composition-Adjusted Wages to Disaster Shocks

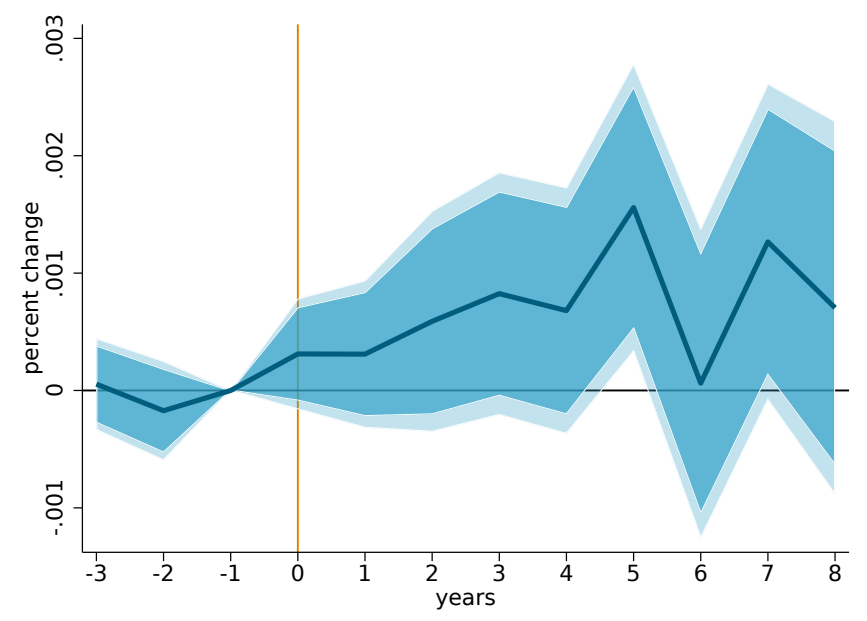

Source: FEMA, SHELDUS, CEPR yearly extracts of the CPS Outgoing Rotation Group micro-data, and the Census Bureau's County Business Patterns.

Note: Figure shows the impulse response function from estimating equation (1) where the dependent variable is an estimate of what the mean wage would be if the local wage composition is applied to the national wage rates. The inner shaded regions indicate the 90 percent confidence intervals, and the lighter outer shaded regions indicate the 95 percent confidence intervals. Standard errors are clustered at the county and at the state-by-time levels. 
Figure A8: Impulse Responses of Migration to Disaster Shocks

(a) In-Migration - All Disasters

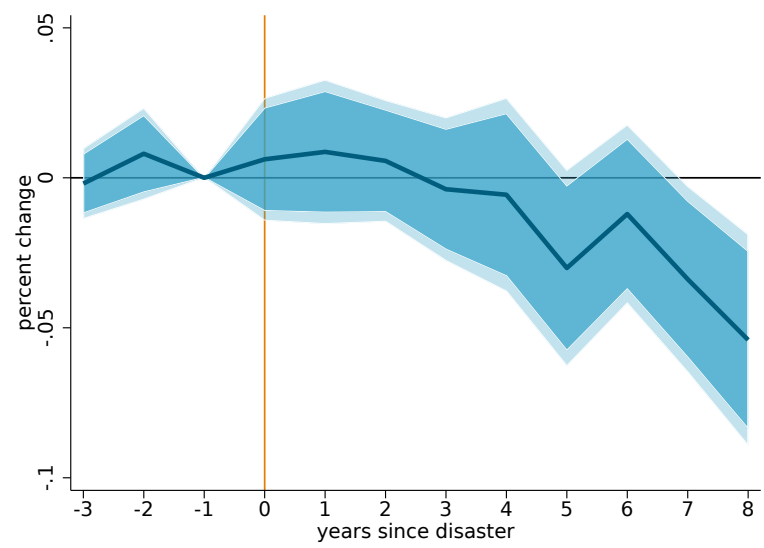

(c) In-Migration - By Disaster Severity

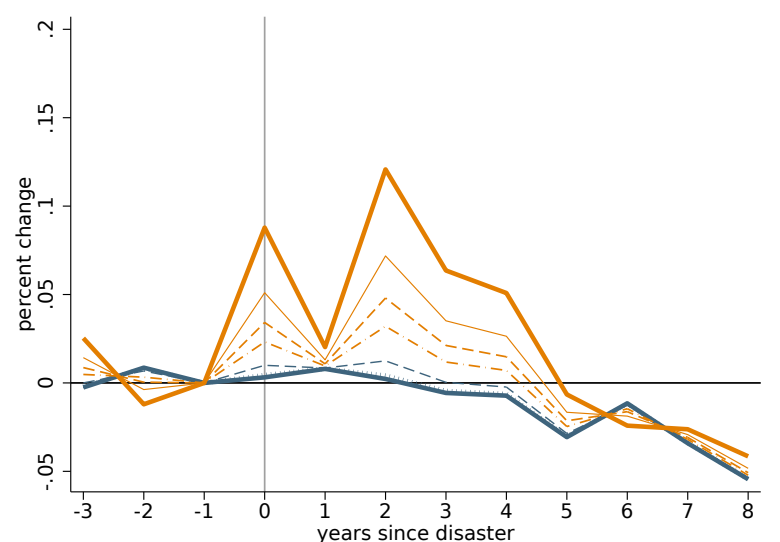

(b) Out-Migration - All Disasters

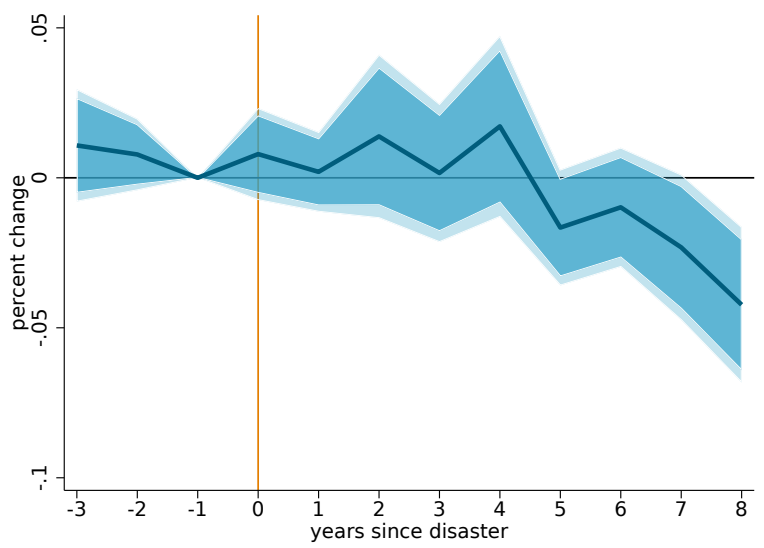

(d) Out-Migration - By Disaster Severity

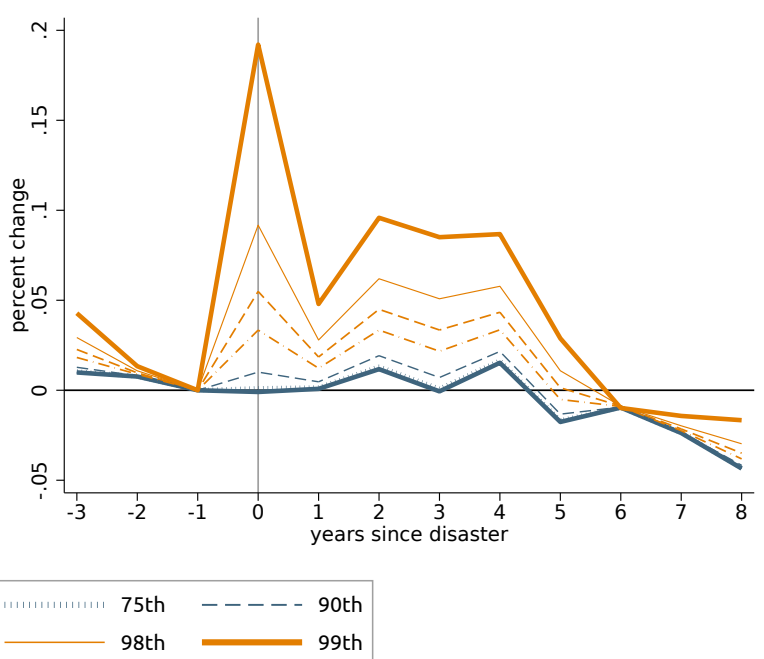

Source: FEMA, SHELDUS, BLS, Census, BEA, and CoreLogic.

Note: Plots (a) and (b) show the impulse response functions (IRFs) from estimating equation (1), where the shaded regions indicate the 90 and 95 percent confidence intervals. Standard errors are clustered at the county and at the state-by-time levels. Plots (c) and (d) show the IRFs from estimating equation (4), where the percentile lines reflect the model predictions given the per capita damage distributions for all county-month observations with FEMA disaster declarations. All variables are observed at the county level and modeled as differences in $\operatorname{logs}$ between the indicated horizon $(h)$ and the period before the disaster $(-1)$. 
Figure A9: Impulse Responses of Government Transfers and Insurance Payouts to Disaster Shocks

(a) FEMA Individual and Household Program Aid

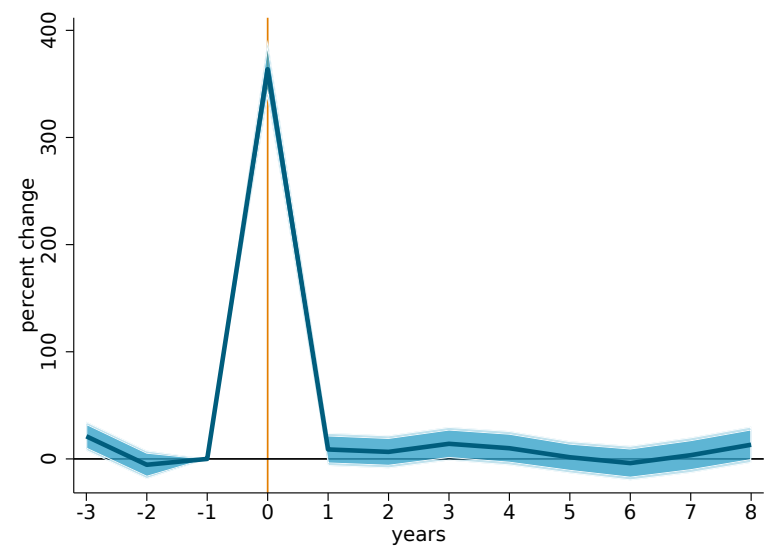

(c) National Flood Insurance Program Payouts

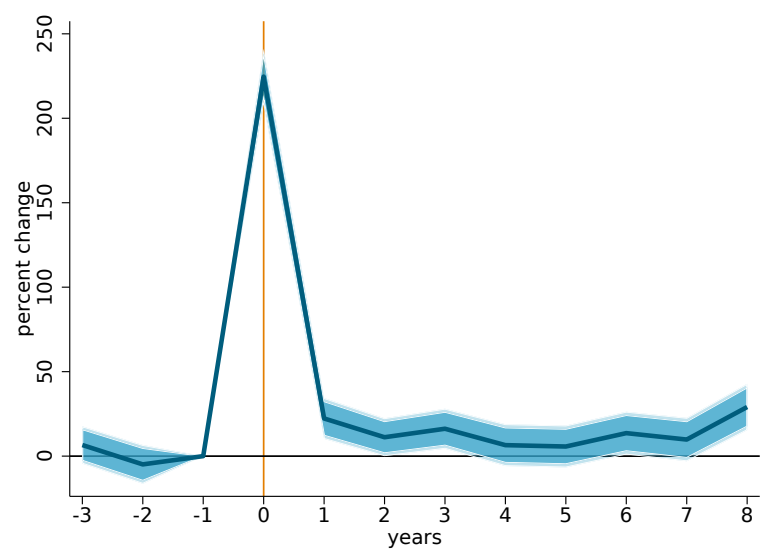

(e) Income Maintenance Transfer Income

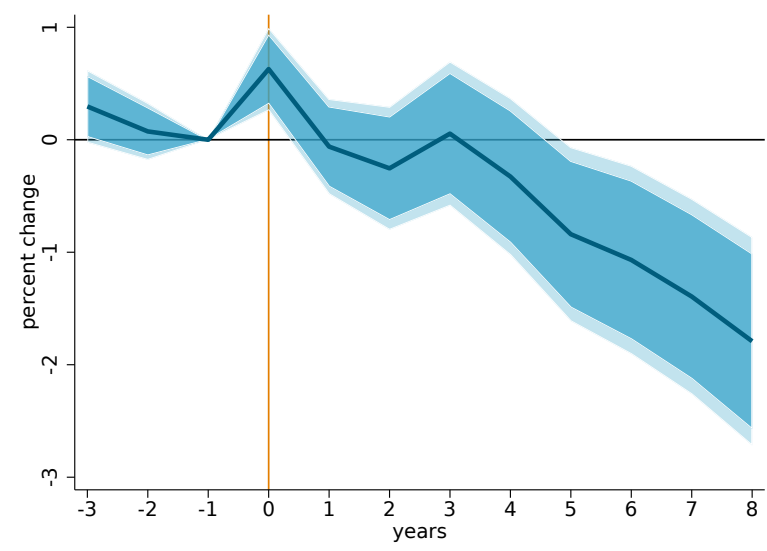

(b) Small Business Admin. Disaster Loans

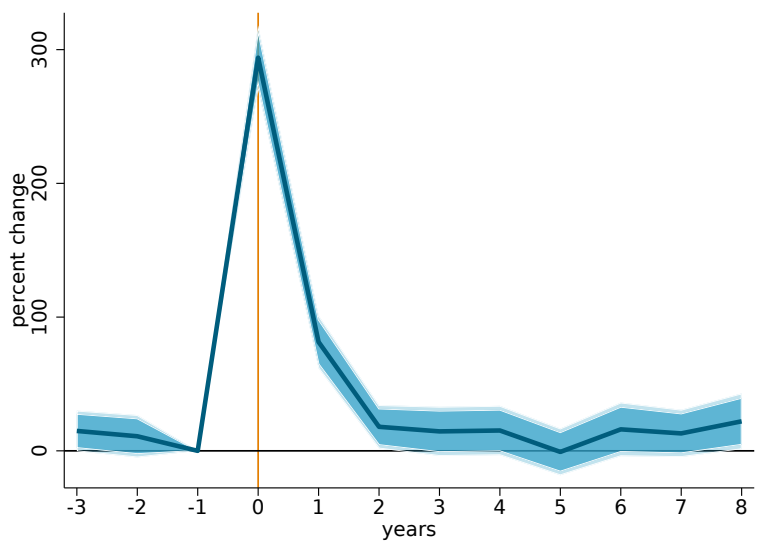

(d) All Government Transfer Income

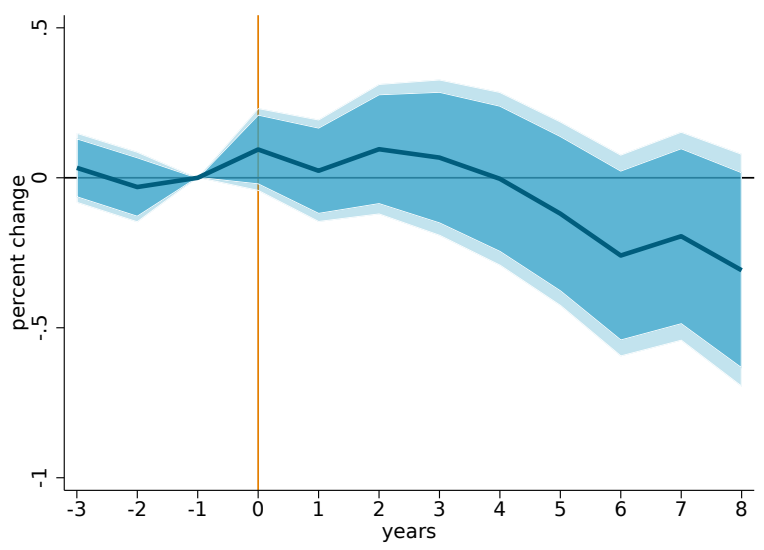

(f) Unemployment Insurance Transfer Income

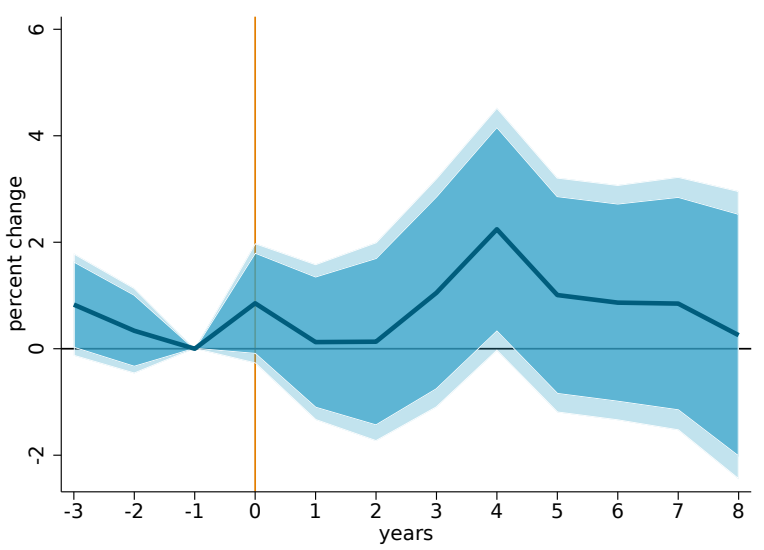

Source: Federal Emergency Management Agency (FEMA), SHELDUS, Small Business Administration (SBA), FEMA National Flood Insurance Program (NFIP) Redacted Claims data, Census, and BEA.

Note: These plots show the impulse response functions for alternative types of government transfers and insurance payouts from estimating equation (1), where the shaded regions indicate the 90 and 95 percent confidence intervals. Standard errors are clustered at the county and at the state-by-time levels. Outcomes variables are observed at the county level, measured in real $(2017 \$)$ per capita units, and modeled as differences in logs between the indicated horizon $(h)$ and the period before the disaster $(-1)$. 
Figure A10: Wage \& Salary Income (per capita)

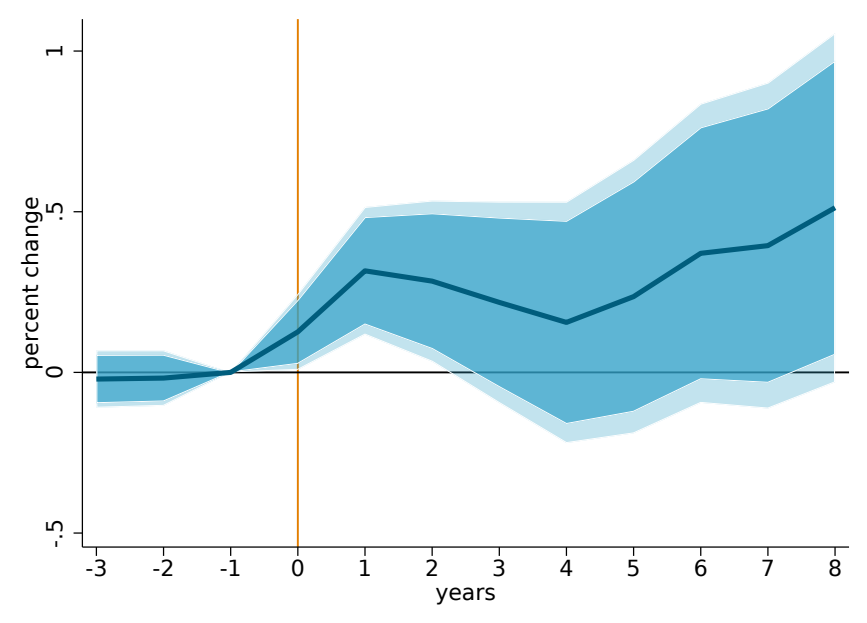

Source: FEMA, SHELDUS, Census, and BEA.

Note: This plots show the impulse response functions from estimating equation (1), where the inner shaded regions indicate the 90 percent confidence intervals and the lighter outer shaded regions indicate the 95 percent confidence intervals. Standard errors are clustered at the county and at the state-by-time levels. Wage \& salary income is observed at the county level and modeled as differences in logs between the indicated horizon $(h)$ and the period before the disaster $(-1)$. 
Figure A11: Heterogeneous Impulse Responses of Personal Income Per Capita to Hurricane Disasters By Severity

\section{Severity Alternately Measured by Damages or by Wind Speed}

(a) Severity measured by Damages p.c.

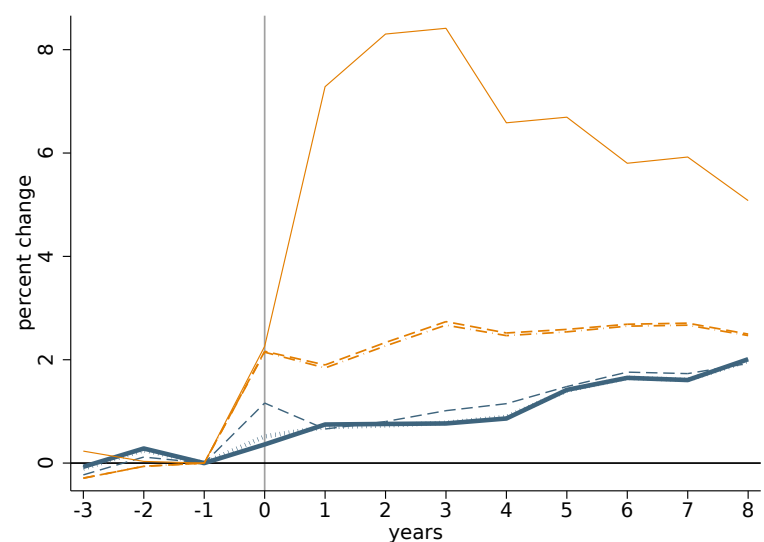

(b) Severity measured by Wind Speed

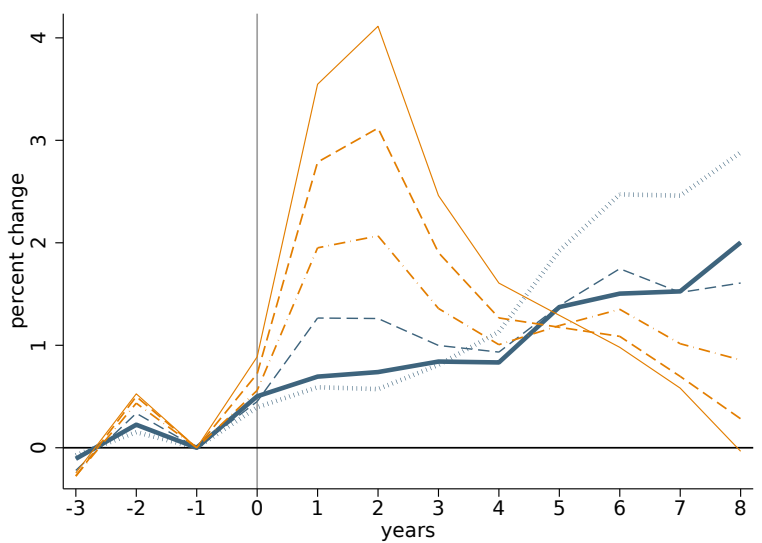

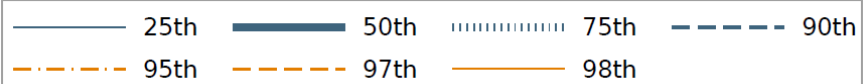

Source: FEMA, SHELDUS, Census, BEA, Anderson et al (2020).

Note: This figure shows the impulse response functions from estimating equation (4) where the treatment variable is a hurricane dummy variable and an indicator variable for non-hurricane disasters is added to the set of controls. Severity (s) is measured by damages p.c. in panel (a) and by wind speed in panel (b). Percentiles are from distribution over all hurricanes in the sample with positive damages. See text for further details.

Figure A12: Personal Income Per Capita in New Orleans Before and After Hurricane Katrina

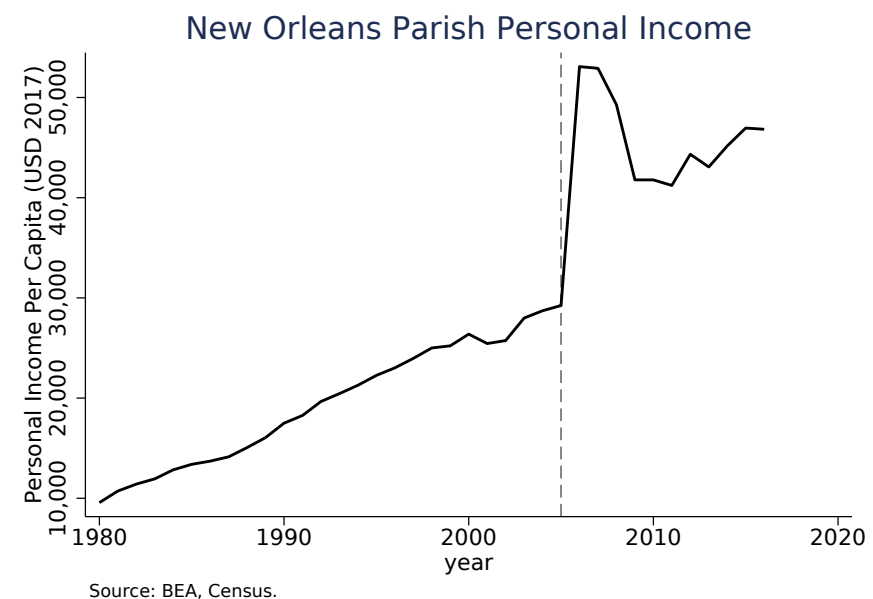

Source: BEA and Census.

Note: Vertical red line indicates 2005, the year of Hurricane Katrina. 
Figure A13: Distribution of Per Capita County Damages by Disaster Type

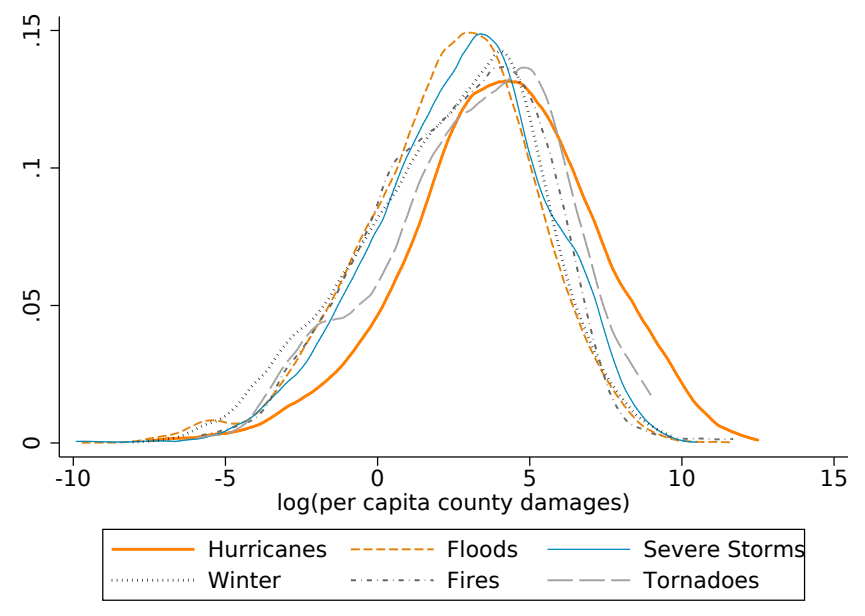

Source: FEMA, SHELDUS.

Note: The y-axis shows density and not frequency. 
Figure A14: Spatial Lags in 1988
(a) Own-County Disasters
(b) $<199$ miles

Counties with disasters in 1988

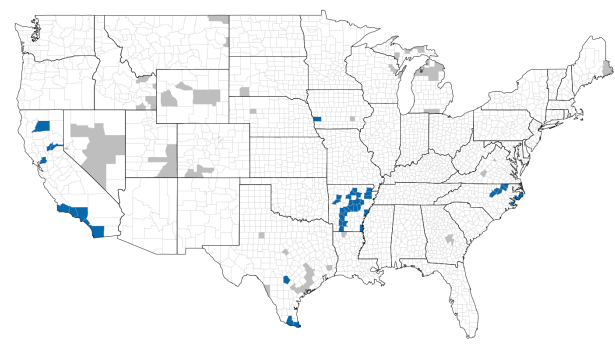

(c) 200-399 miles
0 - 199 mile population share with disasters in 1988

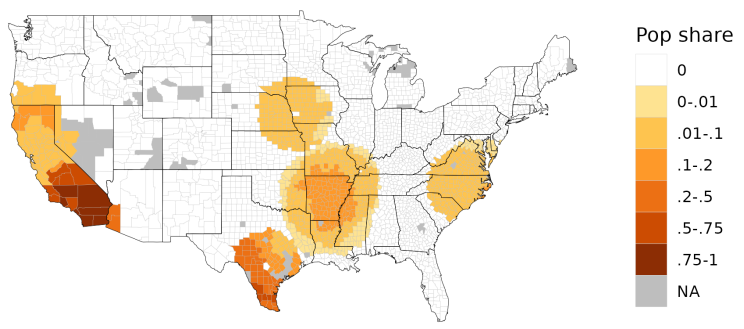

(d) 400-599 miles

400 - 599 mile population share with disasters in 1988

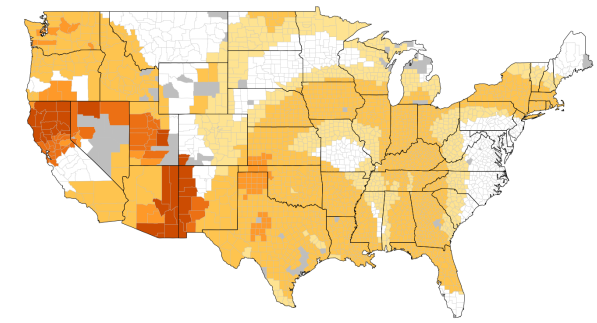

Pop share

0

$0-.01$

$.01-1$

$.1-.2$

$.2-.5$

$5-.75$

Source: FEMA, SHELDUS.

Note: Using 1988 as an example, panel (a) depicts the counties that received major disaster declarations from FEMA with positive damages in SHELDUS. Panels (b)-(c) depict the share of population within each band (50-199, 200-399, and 400-599 miles) of a given county that had disaster declarations with damages. Darker shading in panels (b)-(d) indicate a higher population share. 
Figure A15: State-Level Impacts of Disasters on Personal Income (p.c.) with Disasters Differentiated by Geographic Breadth
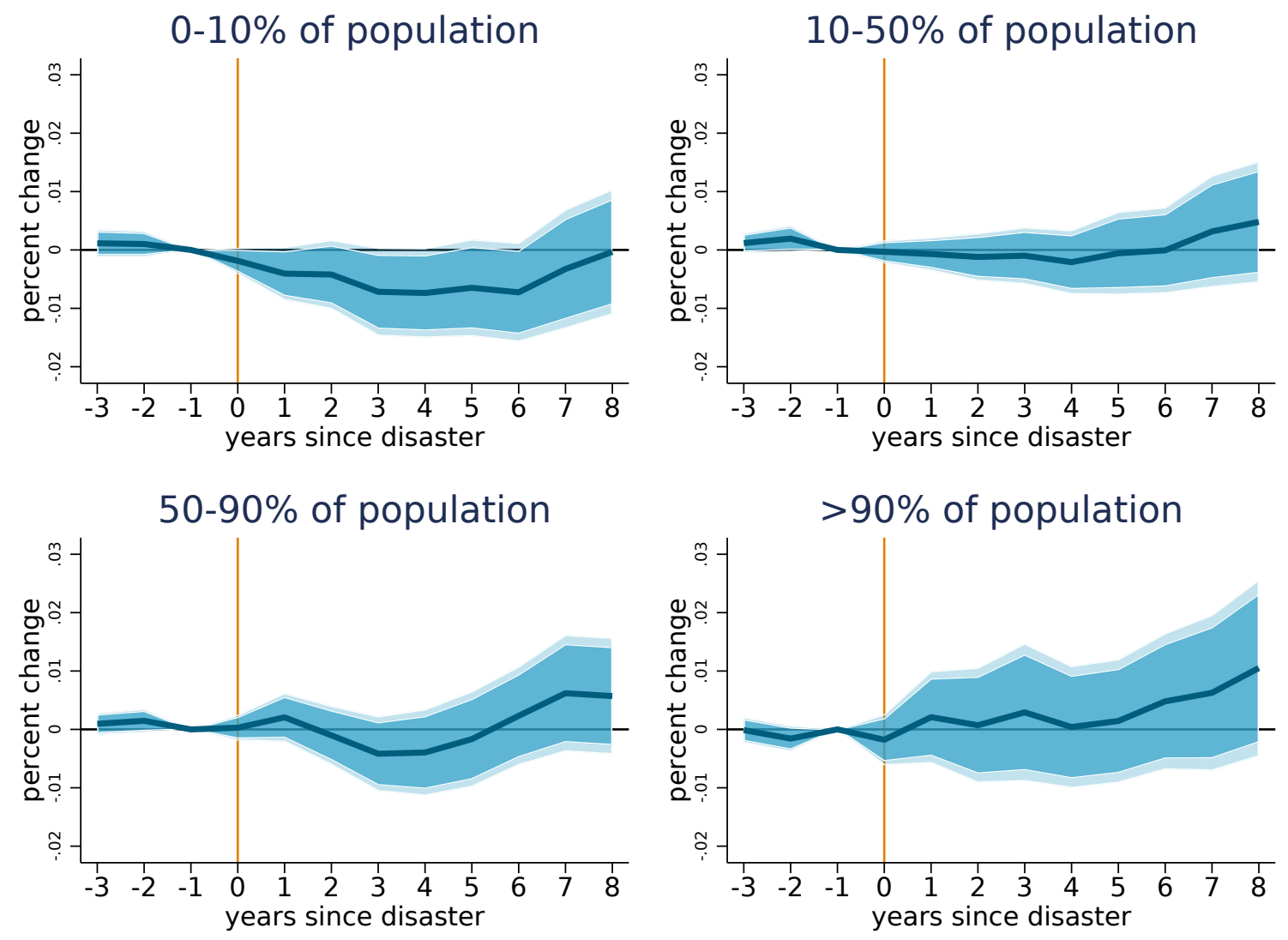

Source: FEMA, SHELDUS, BLS, Census, BEA, and CoreLogic.

Note: This figure shows the impulse response functions from estimating equation (1) on state-aggregated data, but allowing the coefficient on the disaster treatment variable to vary across four geographic breadth indicator variables. These variables indicate what quartile the disaster falls in of the distribution of the share of the state's population in a county hit with a disaster (with positive damages), conditional on having a disaster. The inner shaded regions indicate the 90 percent confidence intervals, and the lighter outer shaded regions indicate the 95 percent confidence intervals. Personal income is aggregated at the state level and modeled as differences in logs between the indicated horizon $(h)$ and the period before the disaster $(-1)$. Standard errors are clustered at the state level. 


\section{Online Appendix B - Not For Publication \\ Additional Details on Data Construction}

\section{B1. Poverty Data}

The county-level poverty rate data come from the Census Bureau's Small Area Income and Poverty Estimates (SAIPE) program and cover the years 1989, 1993, 1995, and 1997-2020. We fill in the missing years for each county via linear interpolation between adjacent years. We expect the resulting measurement error to be systematically unrelated to disaster occurrence and hence expect it to inflate standard errors but not to introduce bias. ${ }^{1}$

\section{B2. Data for Industry Mix-Implied Wages}

We construct a measure of industry mix in order to assess how disasters impact the industry composition of a county's workforce. Specifically, we construct a variable measuring each county's expected average wage in a given year based only on its industry composition:

$$
w_{c t}^{\text {pred }}=\sum_{j} s_{c j t} w_{j t} ; \quad \sum_{j} s_{c j t}=1
$$

where $s_{c j t}$ is the employment in industry $j$ in county $c$ in year $t$ and $w_{j t}$ is the national mean hourly wage in industry $j$ in year $t$.

Data on $s_{c j t}$ come from the Census Bureau's County Business Patterns (CBP) data. We use Eckert, et al. (2021)'s version of the CBP data, which exploits various adding-up constraints in the raw data to fill in missing values. It imputes some missing values in the raw CBP data by exploiting cross-county (within industry) and cross-industry (within county) totals and adding up constraints. To further minimize missing values, we use the "major sector" NAICS industry level rather than a finer level of industry categorization.

We calculate $w_{j t}$ as the mean hourly wage by NAICS major sector across individuals using the CEPR yearly extracts of the CPS Outgoing Rotation Group micro-data. ${ }^{2}$

\footnotetext{
${ }^{1}$ Using only 1997-2020 results in too short a sample to estimate the full dynamic pattern from $h=-3$ to 8 with rea sonable precision.

${ }^{2}$ Using the median wa ge yields qualitatively similar results to the mean.
} 


\section{B3. Government Transfer and Loan Data}

We use data on SBA disaster loans for fiscal years 2001 through 2017 from the SBA website. Data for years from 1989 through 2000 came from Bondonio and Greenbaum (2018) and were generously provided by Robert Greenbaum. The data provide dollar amounts of disbursements of SBA disaster loans, separately for householdsand for businesses, by county and fiscal year. We use data on county level IHP payments going back to 1990 that we obtained from FEMA via FOIA request.

We use the Federal Insurance \& Mitigation Administration National Flood Insurance Program (FIMA NFIP) Redacted Claims Dataset (available at https://www.fema.gov/medialibrary/assets/documents/180374) to calculate NFIP payments associated with floods occurring each month in each county. Although we are able to observe the date of the incident to associate the payment amounts with our disaster observations, we are unable to observe when the payments are actually made.

\section{B4. Hurricane Wind Speed Data}

For hurricane wind speed data, we use county level data made available via Anderson et al (2020a) and Anderson et al (2020b) using the U.S. National Hurricane Center's Best Track Atlantic hurricane database (HURDAT2.) We use versions 0.1.1 and 0.1.0 of R packages hurricaneexposure and hurricaneexposuredata, respectively. Our wind speed measure is the highest maximum sustained wind speed that has a duration of at least 10 minutes. We only include the observed wind speed for a county and event if the maximum gust is at least 64 knots, the maximum sustained wind is at least 50 knots, the daily precipitation is at least 50 $\mathrm{mm}$ (about 2 inches), or the total precipitation over the five-day period is at least $200 \mathrm{~mm}$ (about 8 inches).

\section{B5. Migration Data}

We use IRS Statistics on Income (SOI) data on gross migration flows into and out of each county from 1995 to $2017 .{ }^{3}$ In-migration to a county in a given year is defined as the number of taxpayers (roughly equivalent to households) filing taxes in that county in that year but that filed in a different county in the prior year. Out-migration from a county in a given year is defined as

${ }^{3}$ https://www.irs.gov/statistics/soi-tax-stats-migration-data 
the number of taxpayers (roughly equivalent to households) that filed taxes in that county in the prior year but that filed taxes in a different county in the current year. The IRS does not disclose migration flows of less than 10. In those cases, we treat the flow as a zero. These zeros are relatively rare, occurring for $0.6 \%$ of observations for in-flows and $0.5 \%$ for out-flows.

\section{References}

Anderson B, Yan M, Ferreri J, Crosson W, Al-Hamdan M, Schumacher A and Eddelbuettel D (2020). hurricaneexposure: Explore and Map County-Level Hurricane Exposure in the United States. R package version 0.1.1, <URL: http://CRAN.Rproject.org/package $=$ hurricaneexposure $>$.

Anderson B, Schumacher A, Crosson W, Al-Hamdan M, Yan M, Ferreri J, Chen Z, Quiring S and Guikema S (2020). hurricaneexposuredata: Data Characterizing Exposure to Hurricanes in United States Counties. R package version 0.1.0, $<\mathrm{URL}$ : https://github.com/geanders/hurricaneexposuredata>.

Bondonio, Daniele, and Robert T. Greenbaum (2018). "Natural Disasters and Relief Assistance: Empirical Evidence on the Resilience of U.S. Counties using Dynamic Propensity Score Matching", 